\title{
MASS AND SPIN RENORMALIZATION IN LORENTZ ELECTRODYNAMICS
}

\author{
Walter APPEL* and Michael K.-H. KIESSLING \\ Department of Mathematics, Rutgers University \\ 110 Frelinghuysen Rd., PISCATAWAY, NJ 08854, USA \\ * On leave from: Laboratoire de Physique \\ Unité de recherche 1325 associée au CNRS \\ École normale supérieure de Lyon \\ 46 allée d'Italie, 69364 LYON Cedex 07 France
}

\section{Revised version of October 04, 2000. Typeset with $\mathrm{HT}_{\mathrm{E}} \mathrm{X}$ on:}

October 27, 2018

\begin{abstract}
Lorentz' objection to the Uhlenbeck-Goudsmit proposal of a spin magnetic moment of the electron, namely that the electron's equatorial rotation speed would exceed the speed of light by a factor $\approx 10$, mutated into an objection against Lorentz electrodynamics (LED) itself when the spin magnetic moment became established. However, Lorentz' renormalization calculation, based on the early $20^{\text {th }}$ century's notion of a purely electromagnetic electron, does not qualify as proper from a modern perspective. This paper shows that renormalization treated properly does lead to a mathematically consistent and physically viable LED. A new, relativistically covariant massive LED is presented in which the bare particle has a finite positive bare rest mass and moment of inertia. The particle's electromagnetic self-interaction renormalizes its mass and spin. Most crucially, the renormalized particle is a soliton: after any scattering process its rest mass and spin magnitude are dynamically restored to their pre-scattering values. This guarantees that "an electron remains an electron," poetically speaking. A renormalization flow study of the limit of vanishing bare rest mass is conducted for this model. This limit yields a purely electromagnetic classical field theory with ultra-violet cutoff at about the electron's Compton wavelength! The renormalized limit model matches the empirical electron data as orderly as one can hope for at the level of Lorentz theory. In particular, no superluminal equatorial speeds occur.
\end{abstract}

(c) (2000) The authors. Reproduction for non-commercial purposes of this article, in its entirety and by any means, is permitted. 


\section{Introduction}

The roots of the mass renormalization program of quantum electrodynamics (QED) trace back to the late $19^{\text {th }}$ and early $20^{\text {th }}$ centuries' notion that the electron's inertia has a purely electromagnetic origin. This notion was central to the pre-quantum attempts of the time to construct a consistent electromagnetic theory of matter and radiation that would unify electrodynamics and mechanics, now known as classical electron theory. When Lorentz proposed that Abraham's [1], 2] purely electromagnetic, strictly rigid spherical electron (see Appendix A.2) had to be replaced by an electron that undergoes the Lorentz-Fitzgerald contraction, the laws of special relativity began to take shape in the papers of Lorentz [3], and Poincaré [4]; see [5] for an excellent account of the historical developments. Ironically, special relativity and renormalization later contributed to the demise of relativistic Lorentz electrodynamics (LED) more than quantum mechanics. Of course, quantum mechanics removed LED from the list of contenders for a truly fundamental electromagnetic theory of matter and radiation, but it did not jeopardize the viability of LED as a physical theory in the classical limit. After all, Bohr's correspondence principle was undisputed in the quest for the fundamental quantum theory, and as such one would not have been, nor would one be unhappy with a consistent classical microscopic relativistic theory of electromagnetic matter and radiation that could serve as the empirically correct classical limit of a consistent quantum theory. Special relativity on the other hand had emancipated itself in the works of Einstein [6, 7] and Minkowski [8] as a theory of spacetime structure independent of LED or any other particular matter model [5]. Thus, when Lorentz objected to the Uhlenbeck-Goudsmit [9] proposal of an electron spin magnetic moment by pointing out that LED would forbid the required magnitude of about one Bohr magneton because the purely electromagnetic electron would have to reach an impossible equatorial rotation speed of about 10 times the speed of light (see [10], p. 35), he had furnished an argument that — eventually — weighed heavily against the physical sensibility of LED itself, and which has persisted ever since.

Judged from our modern perspective on mass renormalization [11, 12, the purely electromagnetic calculations by Lorentz [3, 13] and his contemporaries do, however, not qualify as proper. A proper renormalization analysis would investigate the 'flow' toward vanishing bare rest mass for a consistent relativistic LED with positive bare rest mass ('massive' LED) in which the bare particle parameters of the model can be chosen such that the particle charge, the particle spin magnetic moment, and the renormalized particle mass match the empirical electron data without involving superluminal gyration speeds of the model electron. If the limit of vanishing bare rest mass exists, this limit would define a renormalized LED which in a sense completes Lorentz' program of a purely electromagnetic electron theory, and whose physical viability is no longer objectable to on basis of the superluminal arguments that grounded the original Lorentz theory.

In this paper we will show that such a properly renormalized approach to the Lorentz program of electrodynamics does indeed lead to a satisfactory Lorentz electrodynamics. For simplicity we consider only a single Lorentz electron coupled to the Maxwell-Lorentz fields in the whole spacetime. The extension to a many particle theory does not pose any difficulties.

We first present a novel, manifestly Lorentz-covariant massive LED which displays several crucial features expected of a realistic, consistent classical electrodynamics. In particular: 
- the dynamical equations constitute a Cauchy problem for the evolution of the physical state of massive LED;

- scattering does not change the nature of the renormalized particle, which moves as a soliton;

- the parameters of the bare particle can be chosen such that charge, magnetic moment, and renormalized mass of the 'free' particle can be matched to the physical electron data without involving superluminal gyration speeds.

We then study the renormalization flow to vanishing bare rest mass of our empirically matched massive LED. The 'renormalized LED' which emerges in the limit is characterized by the following additional features:

- the equatorial gyration speed reaches the speed of light $c$ precisely in the limit of vanishing bare rest mass so that the bare gyrational mass converges to a nonvanishing contribution. It is fitting to call this a 'photonic' mass;

- the renormalized spin magnitude is a derived property in our model. It turns out to be $3 \hbar / 2$ rather than $\hbar / 2$, with corrections of order $\alpha$ (Sommerfeld's fine structure constant), and we have $g \approx 2 / 3$ rather than $g \approx g_{\mathrm{QM}} \stackrel{\text { def }}{=} 2$ or $g \approx g_{\mathrm{Cl}} \stackrel{\text { def }}{=} 1$.

- our renormalized LED is, strictly speaking, a classical field theory with ultraviolet cutoff corresponding to the physical electron's Compton length!

It is not unexpected that in our classical theory the relation between spin and magnetic moment holds with a Landé factor $g \not 2$. On the other hand, since we operate here with a minimalist model, it is not a priori clear whether a slightly more intelligently constructed classical model would not account also for the correct $g \approx 2$. In any event, the issue of the $g$ factor should not be confused with the superluminal speed problem of Lorentz.

Among the many physically correct features of the model, the truly rewarding one is its soliton dynamics. Without it the rest mass, magnetic moment and spin of the renormalized particle would typically be altered by each scattering process, and the theory would then simply fail to account for the classical motion of electrons and their electromagnetic fields.

Another gratifying feature is the size of the renormalized, purely electromagnetic model electron, whose radius comes out to 1.5 physical electron Compton lengths, about 200 times larger than the somewhat misnamed "classical electron radius" $e^{2} / m c^{2}$. Our renormalized LED is thus intrinsically equipped with the ultraviolet cutoff at which heuristic discussions [14 have traditionally set the spatial limit of applicability of classical electrodynamics as a physical theory.

Remarkable is furthermore that the Cauchy problem for the state evolution bears a certain resemblance to the problem of motion of a black hole in general relativity. Unexpectedly, massive LED thus provides a playground for this much harder problem of general relativity.

Our model owes much to the monumental work of Nodvik [15]. However, although in Nodvik's Lorentz-covariant massive LED one can match the particle parameters to the empirical electron data for charge, mass, and magnetic moment without encountering superluminal 
gyration speeds, one can do this only for sufficiently large bare rest mass. More specifically, the renormalization flow toward smaller bare rest mass terminates at about $1 / 2$ of the empirical electron mass, i.e. before reaching the purely electromagnetic limit of vanishing bare rest mass, and its spin is then merely about $10^{-3} \hbar$. In addition to this deficiency, Nodvik's massive LED suffers from a serious dynamical defect which it shares with the purely electromagnetic models of Abraham and Lorentz; namely, the initial value problem is singular. Interestingly, both deficiencies of Nodvik's massive LED are caused by his choice of bare rest mass distribution, which concentrates all the bare inertia at the center of the particle so that its bare moment of inertia vanishes. By endowing the particle with a bare rest mass distribution that gives it a strictly positive bare moment of inertia our massive LED overcomes both deficiencies and, in this sense, improves decisively on Nodvik's massive LED.

The presentation of our results is organized as follows. To begin with, in section 2 we stipulate the relativistic notation used in this paper. In section 3 we discuss the relativistic kinematical prerequisites of the model. Massive LED is defined by a Lorentz invariant action functional that 'minimally couples' the dynamics of the bare particle and the electromagnetic fields. We first formulate the relativistic mechanics of a gyrating bare particle for given Minkowski force and torque of unspecified origin (section 4) and then, in section 5, present Nodvik's [15] covariant Maxwell-Lorentz equations which determine the electromagnetic fields also in our model, given the kinematical history of the charged particle. Our action functional for massive LED is introduced in section 6, where we also discuss its symmetries and associated conservation laws. The dynamical equations of our massive LED are presented in section 7 . In section 8 we address the Cauchy problem for the state evolution. The scattering problem is discussed in section 9 , where we will see that the renormalized particle behaves like a soliton. In section 10 the renormalization group flow toward vanishing bare rest mass is studied, in detail for the stationary particle, and in an outline for the dynamical particle. We conclude the main body of our paper in section 11 with a summary and an outlook on follow-up work and open problems. In particular, we also speculate about possible implications of our results in the quest for a consistent QED.

In order not to interrupt the flow of the presentation, some technical material adapted from [15], e.g. the derivations of the Euler-Lagrange equations, has been relegated to Appendix A.1. To facilitate the comparison with Nodvik's model [15], Appendix A.2 contains a brief discussion of the point mass limit in which our massive LED formally reduces to the massive LED of Nodvik [15]. In particular, we explain that this limit is singular and severely overdetermines the initial value problem. Incidentally, a similar assessment holds for Abraham's [1, 2] purely electromagnetic versions of semi-relativistic LED with and without spin. Since the ill-posedness of the initial value problem for the purely electromagnetic Abraham model is itself a new observation, Appendix A.3 gives a concise summary of the semi-relativistic massive LED and its purely electromagnetic, singular limit. There we start with the massive model with spin and show next that the more familiar semi-relativistic massive 'LED without spin' actually is a theory with spin in 'hidden' form, obtained from the massive model with spin in a non-singular limit. We then address the singular purely electromagnetic Abraham limit. A fair list of references has been selected from the vast pertinent literature. 


\section{Minkowski space: vectors, tensors, and all that}

Various equivalent conventions for the Minkowski space formalism exist in the literature. By and large, we here follow the conventions of the book by Misner, Thorne and Wheeler [16]. However, since the model is essentially a flat space model, we will not introduce forms. Instead, throughout this work we use four-vectors and tensors constructed from them.

Thus, abstract Minkowski space $\mathbb{M}^{4}$ is a flat four-dimensional pseudo-Riemannian manifold with Lorentzian metric of signature +2 . The invariance group acting on Minkowski space is the inhomogeneous Poincaré group; its homogeneous subgroup is called the Lorentz group; for good discussions, see [17, 18]. We can choose any point $P \in \mathbb{M}^{4}$ and identify $\mathbb{M}^{4}$ with the tangent space $\mathbb{T}_{P}\left(\mathbb{M}^{4}\right)$ at $P$. A tetrad $\mathcal{F}_{\mathrm{L}}=\left\{\boldsymbol{e}_{0}, \boldsymbol{e}_{1}, \boldsymbol{e}_{2}, \boldsymbol{e}_{3}\right\}$ of fixed unit four-vectors in $\mathbb{T}_{P}\left(\mathbb{M}^{4}\right)$ that satisfy the elementary inner product rules

$$
\boldsymbol{e}_{\mu} \cdot \boldsymbol{e}_{\nu}=\left\{\begin{aligned}
-1 & \text { for } \mu=\nu=0 \\
1 & \text { for } \mu=\nu>0 \\
0 & \text { for } \mu \neq \nu
\end{aligned}\right.
$$

is a basis for $\mathbb{T}_{P}\left(\mathbb{M}^{4}\right)$, called a Lorentz frame. With respect to a Lorentz frame, any realvalued vector $\mathbf{v} \in \mathbb{T}_{P}\left(\mathbb{M}^{4}\right)$ has the representation

$$
\mathbf{v}=\sum_{\mu=0}^{3} v^{\mu} \boldsymbol{e}_{\mu}
$$

with $v^{\mu} \in \mathbb{R}$ given by $v^{0}=-\mathbf{v} \cdot \boldsymbol{e}_{0}$ and by $v^{\mu}=\mathbf{V} \cdot \boldsymbol{e}_{\mu}$ for $\mu=1,2,3$. Therefore, $\mathbb{T}_{P}\left(\mathbb{M}^{4}\right)$ can be identified with $\mathbb{R}^{1,3}$, the set of ordered real 4-tuples $\mathbf{V}=\left(v^{0}, v^{1}, v^{2}, v^{3}\right)$, which is a global coordinate chart for $\mathbb{M}^{4}$.

To emphasize spacetime among the various physical realizations of abstract Minkowski space, spacetime points will be denoted by $E$ and called events, and their four-vector representation w.r.t. $\mathcal{F}_{\mathrm{L}}$ will be denoted as $\boldsymbol{x}$ or $\boldsymbol{y}$ etc. The coordinates of $\mathbf{x}$ in a Lorentz frame $\mathcal{F}_{\mathrm{L}}$ are sometimes grouped as

$$
\mathbf{x}=(c t, \boldsymbol{x}),
$$

where $\boldsymbol{x}=\left(x^{1}, x^{2}, x^{3}\right)$ is called a 'point in space,' and $t=x^{0} / c$ an 'instant of time,' with respect to the given Lorentz frame. Here, $c$ is the speed of light in vacuo. Event four-vectors $\mathbf{x}$ are classified into spacelike, lightlike, and timelike according as $\mathbf{x} \cdot \mathbf{x}>0, \mathbf{x} \cdot \mathbf{x}=0$, or $\mathbf{x} \cdot \mathbf{x}<0$, respectively, where the inner product of any two event four-vectors $\mathbf{x}$ and $\boldsymbol{y}$ is evaluated using (2.2), distributivity and the elementary inner product rules (2.1). A spacelike four-vector is connected through an orbit of the Lorentz group with a four-vector of the form $(0, \boldsymbol{x})$, a timelike one with a four-vector of the form $(c t, \mathbf{0})$. The lightlike vectors form the 'light cone,' a double cone in $\mathbb{R}^{1,3}$ which separates the spacelike from the timelike four-vectors. This classification is then carried over to four-vectors $\mathbf{v}$ in abstract Minkowski space.

For convenience, $\mathbf{v} \cdot \boldsymbol{v}$ will sometimes be abbreviated thus,

$$
\mathbf{v} \cdot \mathbf{v}=\|\mathbf{v}\|^{2}
$$


where $\|\mathbf{v}\|$ is the Minkowski 'norm' of $\mathbf{v}$, defined as the principal value of $(\boldsymbol{v} \cdot \mathbf{v})^{1 / 2}$. Notice that $\|\mathbf{v}\|^{2}$ is negative for timelike vectors.

The tensor product between any two four-vectors $\mathbf{a}$ and $\boldsymbol{b}$ is a tensor of rank two, denoted by $\mathbf{a} \otimes \boldsymbol{b}$, and defined by its inner-product action on four-vectors, thus

$$
\begin{aligned}
& (\mathbf{a} \otimes \boldsymbol{b}) \cdot \boldsymbol{c} \stackrel{\text { def }}{=} \mathbf{a}(\boldsymbol{b} \cdot \boldsymbol{c}) \\
& \boldsymbol{c} \cdot(\mathbf{a} \otimes \boldsymbol{b}) \stackrel{\text { def }}{=}(\mathbf{a} \cdot \boldsymbol{c}) \boldsymbol{b}
\end{aligned}
$$

(Notice that our convention here differs from the one used, for instance, in [19].) Given a frame $\left\{\boldsymbol{e}_{\mu}\right\}$, any tensor of rank two, $\mathbf{T}$, can be uniquely written as

$$
\mathbf{T}=\sum_{0 \leqslant \mu, \nu \leqslant 3} T^{\mu \nu} \boldsymbol{e}_{\mu} \otimes \boldsymbol{e}_{\nu}
$$

A rank-two tensor $\mathbf{T}$ for which $T^{\mu \nu}= \pm T^{\nu \mu}$ in (2.7) is called symmetric ( + sign), respectively anti-symmetric ( - sign). For symmetric $(+)$ and anti-symmetric $(-)$ tensors we have

$$
\mathbf{T}_{ \pm} \cdot \mathbf{v}= \pm \mathbf{v} \cdot \mathbf{T}_{ \pm}
$$

but for a general tensor

$$
\mathbf{T}_{\text {general }} \cdot \boldsymbol{v} \neq \pm \boldsymbol{V} \cdot \mathbf{T}_{\text {general }} .
$$

A special example for an anti-symmetric tensor of rank two is the exterior product between two four-vectors, denoted by a wedge-up product $\mathbf{a} \wedge \boldsymbol{b}$, and defined by

$$
\mathbf{a} \wedge \boldsymbol{b} \stackrel{\text { def }}{=} \mathbf{a} \otimes \boldsymbol{b}-\boldsymbol{b} \otimes \mathbf{a} .
$$

Examples for symmetric tensors of rank two are, (i) the symmetrized tensor product between two four-vectors, denoted by a wedge-down product $\mathbf{a} \vee \boldsymbol{b}$, and defined by

$$
\mathbf{a} \vee \boldsymbol{b} \stackrel{\text { def }}{=} \mathbf{a} \otimes \boldsymbol{b}+\boldsymbol{b} \otimes \mathbf{a}
$$

and (ii) the metric tensor

$$
\mathbf{g}=\sum_{0 \leqslant \mu, \nu \leqslant 3} g^{\mu \nu} \boldsymbol{e}_{\mu} \otimes \boldsymbol{e}_{\nu}
$$

with $g^{\mu \nu}=\boldsymbol{e}_{\mu} \cdot \boldsymbol{e}_{\nu}$ given in (2.1). Notice that $\mathbf{g}$ has the same components $g^{\mu \nu}$ in all Lorentz frames because the $\boldsymbol{e}_{\mu} \cdot \boldsymbol{e}_{\nu}$ are Lorentz scalars. Notice in particular also that $\mathbf{g}$ acts as identity on four-vectors, i.e. $\mathbf{g} \cdot \mathbf{v}=\mathbf{v}$.

The (anti-) commutator of any two tensors of rank two, $\mathbf{A}$ and $\mathbf{B}$ is defined as usual,

$$
[\mathbf{A}, \mathbf{B}]_{ \pm} \stackrel{\text { def }}{=} \mathbf{A} \cdot \mathbf{B} \pm \mathbf{B} \cdot \mathbf{A}
$$

The action of tensors on a vector is associative, i.e.

$$
(\mathbf{A} \cdot \mathbf{B}) \cdot \mathbf{v}=\mathbf{A} \cdot(\mathbf{B} \cdot \mathbf{v})
$$


so that

$$
[\mathbf{A}, \mathbf{B}]_{ \pm} \cdot \mathbf{v}=\mathbf{A} \cdot(\mathbf{B} \cdot \mathbf{v}) \pm \mathbf{B} \cdot(\mathbf{A} \cdot \mathbf{v}) .
$$

Warning: In general,

$$
\mathbf{A} \cdot(\mathbf{v} \cdot \mathbf{B}) \neq(\mathbf{A} \cdot \mathbf{v}) \cdot \mathbf{B} \text {. }
$$

The four-trace, or contraction, of a tensor of rank two is given by

$$
\operatorname{Tr} \mathbf{T}=\sum_{0 \leqslant \mu \leqslant 3} g^{\mu \mu} T^{\mu \mu}
$$

In particular, $\operatorname{Tr} \mathbf{g}=4$, while $\operatorname{Tr} \mathbf{A}=0$ for any anti-symmetric tensor $\mathbf{A}$.

For a differentiable function $f(\mathbf{x})$ we denote its four-gradient with respect to the metric $\mathbf{g}$ by $\nabla_{\mathrm{g}} f$. In local Lorentz frame coordinates it is given by

$$
\nabla_{\mathrm{g}} f(\mathbf{x})=\left(-\partial_{x^{0}} f, \boldsymbol{\nabla} f\right) \text {. }
$$

where $\boldsymbol{\nabla}$ is the usual three-gradient. In analogy with the conventional curl, we also define the four-curl of a differentiable four-vector function as the anti-symmetric four tensor function

$$
\boldsymbol{\nabla}_{\mathrm{g}} \wedge \boldsymbol{A}(\mathbf{x})=\sum_{0 \leqslant \mu, \nu, \lambda, \eta \leqslant 3} \varepsilon^{\mu \nu \lambda \eta} \boldsymbol{e}_{\mu} \otimes \boldsymbol{e}_{\nu}\left(\boldsymbol{e}_{\lambda} \cdot \nabla_{\mathrm{g}}\right)\left(\boldsymbol{e}_{\eta} \cdot \boldsymbol{A}\right)
$$

where the $\varepsilon^{\mu \nu \lambda \eta}$ are the entries of the conventional rank-four Levi-Civita tensor. The fourLaplacian with respect to $\mathbf{g}$ is just the (negative of the) d'Alembertian, or wave operator, given by

$$
\Delta_{\mathrm{g}} \stackrel{\text { def }}{=} \nabla_{\mathrm{g}} \cdot \nabla_{\mathrm{g}}=-\square
$$

\section{$3 \quad$ Particle kinematics}

A spherically extended, 'rigidly' gyrating particle in spacetime is kinematically described by a pair of curves in Minkowski space. We shall assume that both curves are of class $C^{2}$.

One curve is the so-called world-line of the particle, a timelike future-oriented curve in spacetime itself. The concept of the world-line is familiar from the relativistic mechanics of point particles. For an extended 'rigid' particle an event on its world-line marks the location of the particle's center in spacetime. The world-line has associated with it an invariant 'arc-length' element $\mathrm{d} \tau$. In units such that the speed of light $c=1$, which henceforth we shall use, this invariant arc-length element becomes the proper-time element. Choosing any particular event $E_{0}$ on the world-line as reference world point and assigning to it the propertime $\tau\left(E_{0}\right)=0$, and stipulating the 'future' direction as the one along which $\mathrm{d} \tau$ increases, then by integrating $\mathrm{d} \tau$ along the world line automatically determines a unique invariant proper-time $\tau(E) \in \mathbb{R}$ for any other event $E$ on the world-line. Since the world-line is timelike future-oriented, its proper-time assignment is one-to-one onto and order-preserving, 
whence serves as a natural parameterization for the world-line. In any particular Lorentz frame $\mathcal{F}_{\mathrm{L}}$, the particle world-line is then given by a mapping $\tau \mapsto \boldsymbol{x}=\boldsymbol{q}(\tau) \in \mathbb{R}^{1,3}$. The proper-time element accordingly is given by $\mathrm{d} \tau=\sqrt{-\mathrm{d} \boldsymbol{x} \cdot \mathrm{d} \boldsymbol{x}}$, where $\mathrm{d} \mathbf{x}$ is taken along the world-line.

With this parameterization of the world-line, the four-velocity of the particle at the event $\boldsymbol{q}(\tau)$ is obtained as $\mathrm{d} \mathbf{x} / \mathrm{d} \tau=\stackrel{\circ}{\boldsymbol{q}}(\tau) \stackrel{\text { def }}{=} \mathbf{u}(\tau)$. The map $\tau \mapsto \mathbf{u}(\tau)$, called 'four-hodograph,' traces out a complicated curve in four-velocity space, located on the future unit hyperboloid which is the genus-zero three-dimensional sub-manifold defined by $\boldsymbol{u} \cdot \boldsymbol{u}=-1$ and $u^{0}>0$. When $\tau \mapsto \mathbf{u}$ is constant, the four-hodograph degenerates into a Minkowski point. We will sometimes group the four-velocity into its time and space components w.r.t. the chosen Lorentz frame, as

$$
\mathbf{u}=(\gamma, \gamma \boldsymbol{v})
$$

where $\boldsymbol{v}$ is the usual three-velocity in the space part of the chosen Lorentz frame, and where

$$
\gamma=\frac{1}{\sqrt{1-|\boldsymbol{v}|^{2}}} .
$$

Finally, by taking the second derivative w.r.t. $\tau$ we obtain the four-acceleration of the particle at the event represented by $\boldsymbol{q}(\tau)$, thus $\mathrm{d}^{2} \boldsymbol{x} / \mathrm{d} \tau^{2}=\stackrel{\circ}{\mathbf{u}}(\tau) \stackrel{\text { def }}{=} \mathbf{a}(\tau)$. The map $\tau \mapsto \mathbf{a}(\tau)$ is a section in the tangent bundle to the future unit hyperboloid of four-velocity space.

The world-line does not contain any kinematical information about the gyration of the extended rigid particle. That information is contained in what may be called the gyrograph of the particle, a spacelike-oriented curve in four angular velocity space, or gyrospace for short. The introduction of gyrospace and the gyrograph map requires the consideration of two non-inertial reference frames:

- the body frame $\mathcal{F}_{\text {body }}$;

- the Fermi-Walker frame $\mathcal{F}_{\mathrm{FW}}$.

The body frame $\mathcal{F}_{\text {body }}$ is a co-moving and co-rotating frame, fixed in the particle. It is given by a tetrad of orthonormal basis vectors $\left\{\tilde{\boldsymbol{e}}_{\mu}\right\}_{\mu=0, \ldots, 3}$, with $\tilde{\boldsymbol{e}}_{0}=\boldsymbol{u}(\tau)$, that satisfy the equation [16]

$$
\frac{\mathrm{d}}{\mathrm{d} \tau} \tilde{\mathbf{e}}_{\mu}=-\boldsymbol{\Omega} \cdot \tilde{\boldsymbol{e}}_{\mu}
$$

where $\Omega$ is the anti-symmetric tensor of the instantaneous rate of four-gyration of the particle's body frame $\mathcal{F}_{\text {body }}$ as seen in the Lorentz frame $\mathcal{F}_{\mathrm{L}}$. The components of four-vectors with respect to $\mathcal{F}_{\text {body }}$ will be marked by primes: $\boldsymbol{x}=\sum_{0 \leqslant \mu \leqslant 3} \tilde{x}^{\mu} \tilde{\boldsymbol{e}}_{\mu}$.

The Fermi-Walker frame $\mathcal{F}_{\mathrm{FW}}$ is the natural co-moving tetrad of orthonormal basis vectors $\left\{\overline{\boldsymbol{e}}_{\mu}\right\}_{\mu=0, \ldots, 3}$, with $\overline{\boldsymbol{e}}_{0}=\mathbf{u}(\tau)$, satisfying the law of Fermi-Walker transport [16,

$$
\frac{\mathrm{d}}{\mathrm{d} \tau} \overline{\boldsymbol{e}}_{\mu}=-\Omega_{\mathrm{FW}} \cdot \overline{\boldsymbol{e}}_{\mu}
$$


where

$$
\Omega_{\mathrm{FW}} \stackrel{\text { def }}{=} \stackrel{\circ}{\boldsymbol{u}} \wedge \boldsymbol{u}
$$

is the anti-symmetric Fermi-Walker tensor. The components of vectors with respect to $\mathcal{F}_{\mathrm{FW}}$ will be marked by overbars, i.e. $\boldsymbol{x}=\sum_{0 \leqslant \mu \leqslant 3} \bar{x}^{\mu} \overline{\boldsymbol{e}}_{\mu}$.

Fermi-Walker transport is a kinematical effect which does not discriminate between objects of different physical characteristics like inertia or charge. The most prominent effect associated with Fermi-Walker transport is the celebrated Thomas precession [20] of the space axes of $\mathcal{F}_{\mathrm{FW}}$ relative to the Lorentz frame $\mathcal{F}_{\mathrm{L}}$, described by the angular velocity threevector [10, 20, 21],

$$
\boldsymbol{\omega}_{\mathrm{T}}=(\gamma-1) \frac{1}{|\boldsymbol{v}|^{2}} \boldsymbol{a} \times \boldsymbol{v} .
$$

Here $\boldsymbol{a}$ is the usual three-acceleration of the origin of $\mathcal{F}_{\mathrm{FW}}$ as seen from $\mathcal{F}_{\mathrm{L}}$, and $\boldsymbol{v}$ its threevelocity.

If the four-gyration of the body frame w.r.t. the Lorentz frame differs from the Thomas precession, one ascribes this difference to the particle's intrinsic four-gyration, which is captured by the anti-symmetric tensor

$$
\Omega-\Omega_{\mathrm{FW}} \stackrel{\text { def }}{=} \Omega_{\mathrm{E}} .
$$

The subscript 'E' here is meant as reference to Euler's monumental analysis of Newtonian gyration of an inert rigid body. Beside being anti-symmetric, the tensor $\Omega_{\mathrm{E}}$ satisfies

$$
\Omega_{\mathrm{E}} \cdot \mathbf{u}=\boldsymbol{O},
$$

so that $\boldsymbol{\Omega}_{\mathrm{E}}$ has only three independent components. It follows that $\boldsymbol{\Omega}_{\mathrm{E}}$ is dual to a spacelike four-vector $\boldsymbol{w}_{\mathrm{E}}$ which satisfies

$$
\Omega_{\mathrm{E}} \cdot \boldsymbol{W}_{\mathrm{E}}=\boldsymbol{O}
$$

and $\boldsymbol{w}_{\mathrm{E}} \cdot \boldsymbol{u}=\boldsymbol{O}$. In $\mathcal{F}_{\mathrm{FW}}, \boldsymbol{w}_{\mathrm{E}}$ is given in the form $\boldsymbol{w}_{\mathrm{E}}=\left(0, \boldsymbol{\omega}_{\mathrm{E}}\right)$, where $\boldsymbol{\omega}_{\mathrm{E}}$ is the usual angular velocity three-vector which is directed along the instantaneous axis of body gyration in the spacelike three-slice of the frame $\mathcal{F}_{\mathrm{FW}}$.

At each instant of proper-time $\tau$, the instantaneous state of intrinsic four-gyration of the particle is captured by the four-vector $\boldsymbol{w}_{\mathrm{E}}(\tau)$. As $\tau \in \mathbb{R}$ varies along the particle's world-line, the map $\tau \mapsto \boldsymbol{w}_{\mathrm{E}}(\tau) \in \mathbb{R}^{1,3}$ traces out the gyrograph of the particle in gyrospace, which is the Minkowski space consisting of all possible four-vectors with the meaning of an $\boldsymbol{w}_{\mathrm{E}}$. The gyrograph is spacelike, since for $\boldsymbol{\omega}_{\mathrm{E}} \neq \mathbf{0}$, we have

$$
-\frac{1}{2} \operatorname{Tr}\left(\boldsymbol{\Omega}_{\mathrm{E}} \cdot \boldsymbol{\Omega}_{\mathrm{E}}\right)=\left\|\boldsymbol{w}_{\mathrm{E}}\right\|^{2}=\left|\boldsymbol{\omega}_{\mathrm{E}}\right|^{2}>0 .
$$

\section{Bare particle mechanics}

Formally stripping the particle from its electromagnetic features leaves one with the bare particle. Our bare particle is characterized by a strictly positive inertia against changes of its translational and gyrational momenta, respectively. 


\subsection{Bare inertia}

In our model, all forms of bare inertia are computed with a compactly supported, spherical 'density' $f_{\mathrm{m}}(||):. \mathbb{R}^{3} \rightarrow \mathbb{R}^{+}$of inert mass of unspecified non-electromagnetic origin. In general, $f_{\mathrm{m}}: \mathbb{R}^{+} \rightarrow \mathbb{R}^{+}$is a measure on $[0, R]$. We demand that $f_{\mathrm{m}}$ generates a strictly positive moment of inertia (see below). For instance, two admissible mass densities are:

- (volume inertia) $f_{\mathrm{m}}(\|\mathbf{x}\|)=m_{\mathrm{b}} \frac{3}{4 \pi R^{3}} \chi_{R}(\|\mathbf{x}\|)$,

- $\left(\right.$ surface inertia) $f_{\mathrm{m}}(\|\mathbf{x}\|)=m_{\mathrm{b}} \frac{1}{4 \pi R^{2}} \delta(\|\mathbf{x}\|-R)$.

Here, $\delta($.$) is the Dirac distribution, and \chi_{R}($.$) the characteristic function of the inter-$ val $[0, R]$ with $R>0$. A fraction of the mass in $f_{\mathrm{m}}$ is allowed to be a point mass, but not all of it. Notice that the complete point mass density $f_{\mathrm{m}}(|\boldsymbol{x}|)=m_{\mathrm{b}}\left(1 / 4 \pi|\boldsymbol{x}|^{2}\right) \delta(|\boldsymbol{x}|)$ violates our condition that $f_{\mathrm{m}}$ must have a strictly positive moment of inertia. The singular limiting case of a point mass density is addressed in Appendix A.2.

The most basic inertia is the bare rest mass, given by

$$
m_{\mathrm{b}}=\int_{\mathbb{R}^{3}} f_{\mathrm{m}}(|\boldsymbol{x}|) \mathrm{d}^{3} x,
$$

where $r=|\boldsymbol{x}|$. Since in general the bare particle is in a state of gyration, its instantaneous effective inert mass in the Fermi-Walker frame is the sum of its bare rest mass and the relativistic kinetic energy associated with its Euler gyration, which we call the bare gyrational mass, a Lorentz scalar given by

$$
\begin{aligned}
& \mathcal{M}_{\mathrm{b}}\left(\left\|\boldsymbol{w}_{\mathrm{E}}\right\|\right) \stackrel{\text { def }}{=} \int_{\mathbb{R}^{1,3}} \frac{1}{\sqrt{1-\left\|\boldsymbol{\Omega}_{\mathrm{E}} \cdot \boldsymbol{x}\right\|^{2}}} f_{\mathrm{m}}(\|\boldsymbol{x}\|) \delta(\boldsymbol{u} \cdot \boldsymbol{x}) \mathrm{d}^{4} \mathbf{x} \\
&=\int_{0}^{R} \frac{1}{\left|\boldsymbol{\omega}_{\mathrm{E}}\right| r} \operatorname{artanh}\left(\left|\boldsymbol{\omega}_{\mathrm{E}}\right| r\right) f_{\mathrm{m}}(r) 4 \pi r^{2} \mathrm{~d} r .
\end{aligned}
$$

The 'integration over a simultaneity space slice' in (4.2) follows the insight of Fermi [22], who used this setup in his solution to the infamous $4 / 3$ problem (see [23, 24, 25, 26, 27, 28, 29, 30] for discussions of the $4 / 3$ problem). In the limit of small inertial angular velocity, $\left|\boldsymbol{\omega}_{\mathrm{E}}\right| \ll 1$, a MacLaurin expansion of (4.3) in powers of $\left|\boldsymbol{\omega}_{\mathrm{E}}\right|$ gives the expected result

$$
\mathcal{M}_{\mathrm{b}}\left(\left|\boldsymbol{\omega}_{\mathrm{E}}\right|\right)=m_{\mathrm{b}}+\frac{1}{2} I_{\mathrm{b}}\left|\boldsymbol{\omega}_{\mathrm{E}}\right|^{2}+O\left(\left|\boldsymbol{\omega}_{\mathrm{E}}\right|^{4}\right)
$$

where

$$
I_{\mathrm{b}}=\frac{2}{3} \int_{\mathbb{R}^{3}}|\boldsymbol{x}|^{2} f_{\mathrm{m}}(|\boldsymbol{x}|) \mathrm{d}^{3} x
$$

is the bare particle's non-relativistic principal moment of inertia. By the spherical symmetry of the particle in any of its rest frames the principal inertias are of course degenerate. The 
corresponding non-relativistic moment-of-inertia three-tensor of the bare particle generalizes to the tensor of the Minkowski inertia (about $\mathbf{q}$ ), given by

$$
\mathbf{I}_{\mathrm{b}}\left(\left\|\boldsymbol{w}_{\mathrm{E}}\right\|\right)=\int_{\mathbb{R}^{1,3}} \frac{\|\mathbf{x}\|^{2} \mathbf{g}-\mathbf{x} \otimes \boldsymbol{x}}{\sqrt{1-\left\|\boldsymbol{\Omega}_{\mathrm{E}}(\tau) \cdot \boldsymbol{x}\right\|^{2}}} f_{\mathrm{m}}(\|\mathbf{x}\|) \delta(\mathbf{u}(\tau) \cdot \boldsymbol{x}) \mathrm{d}^{4} \boldsymbol{x} .
$$

which captures the inertia associated with changes of the state of intrinsic four-gyration of the bare particle.

\subsection{Bare dynamics}

The various inertias acquire their meaning from their role as 'coefficients of resistance' against changes of the bare particle's various Minkowski momenta. The bare particle's four-vector of Minkowski momentum is given by

$$
\boldsymbol{p}_{\mathrm{b}}=\mathcal{M}_{\mathrm{b}} \boldsymbol{u}
$$

and the four-vector of its Minkowski spin by

$$
\mathbf{S}_{\mathrm{b}}=\mathbf{I}_{\mathrm{b}} \cdot \boldsymbol{W}_{\mathrm{E}} \text {. }
$$

For a given Minkowski force $\boldsymbol{f}(\tau)$ and Minkowski torque $\boldsymbol{t}(\tau)$, here of unspecified origin, but satisfying $\boldsymbol{t} \cdot \boldsymbol{u}=\boldsymbol{O}$ and $\boldsymbol{f} \cdot \boldsymbol{u}=\boldsymbol{O}$, the evolution of these momenta is governed by the coupled dynamical equations

$$
\frac{\mathrm{d}}{\mathrm{d} \tau} \boldsymbol{p}_{\mathrm{b}}=\boldsymbol{f}
$$

and

$$
\frac{\mathrm{d}}{\mathrm{d} \tau} \boldsymbol{S}_{\mathrm{b}}+\boldsymbol{\Omega}_{\mathrm{FW}} \cdot \boldsymbol{S}_{\mathrm{b}}=\boldsymbol{t}
$$

Due to the occurrence of $\boldsymbol{q}(\tau)$ in the gyrational mass, these dynamical equations are of second order for $\boldsymbol{q}$ and of first order for $\boldsymbol{w}_{\mathrm{E}}$, so they need to be supplemented with initial data for $\boldsymbol{q}, \mathbf{u}$, and $\boldsymbol{w}_{\mathrm{E}}$. A discussion of these equations is interestingly in itself, but will not be pursued here.

\section{Nodvik's Maxwell-Lorentz equations}

If the charged particle's world-line $\boldsymbol{q}(\tau)$ and gyrograph $\boldsymbol{\Omega}_{\mathrm{E}}(\tau)$ are given, the anti-symmetric electromagnetic tensor field of rank two $\mathbf{F}$ can be computed by solving the linear, relativistic Maxwell-Lorentz equations

$$
\begin{aligned}
& \boldsymbol{\nabla}_{\mathrm{g}} \cdot{ }^{\star} \mathbf{F}=\boldsymbol{O}, \\
& \boldsymbol{\nabla}_{\mathrm{g}} \cdot \mathbf{F}=4 \pi \boldsymbol{J},
\end{aligned}
$$


where ${ }^{\star} \mathbf{F}$ is the (left) Hodge dual of $\mathbf{F}$, and where the covariant charge-current density fourvector field $\boldsymbol{J}$ is of the general form 'charge density times four-velocity.' The specific form of the charge-current density of course depends on the particle model under consideration. Our massive LED inherits the charge-current density from Nodvik's [15] model of a gyrating Lorentz electron, which generalizes the non-relativistic expression for a gyrating, strictly rigid Abraham electron (Appendix A.3) to the manifestly covariant expression

$$
\boldsymbol{J}(\mathbf{x})=\int_{-\infty}^{+\infty} \boldsymbol{U}(\mathbf{x} ; \tau) f_{\mathrm{e}}(\|\mathbf{x}-\boldsymbol{q}(\tau)\|) \delta(\mathbf{u}(\tau) \cdot(\mathbf{x}-\boldsymbol{q}(\tau))) \mathrm{d} \tau
$$

Here,

$$
\mathbf{U}(\mathbf{x} ; \tau)=\mathbf{u}(\tau)-\mathbf{\Omega}(\tau) \cdot(\mathbf{x}-\mathbf{q}(\tau))
$$

is the four-velocity of a particle element located at $\boldsymbol{x}$ in $\mathcal{F}_{\mathrm{L}}$, with $\boldsymbol{\Omega}=\boldsymbol{\Omega}_{\mathrm{FW}}+\boldsymbol{\Omega}_{\mathrm{E}}$, and $f_{\mathrm{e}}(|\cdot|): \mathbb{R}^{3} \rightarrow \mathbb{R}^{-}$is the spherically symmetric electrical charge 'density' (in general a finite measure) of the particle at rest. To avoid infinite electromagnetic energy densities and forces, we request that $f_{\mathrm{e}}$ does not feature any singular Dirac point charge distribution anywhere on its support; more general Dirac distributions are however allowed. For instance, the following two possibilities are admissible:

- $\left(\right.$ volume charge) $f_{\mathrm{e}}(\|\mathbf{x}\|)=-e \frac{3}{4 \pi R^{3}} \chi_{R}(\|\mathbf{x}\|)$,

- $\left(\right.$ surface charge) $f_{\mathrm{e}}(\|\mathbf{x}\|)=-e \frac{1}{4 \pi R^{2}} \delta(\|\mathbf{x}\|-R)$.

Here, $e>0$ is the elementary charge as appropriate for a model of an electron.

Problems with infinite self energies and self forces aside, however, it should be noticed that in the formal limit $f_{\mathrm{e}}(|\boldsymbol{x}|) \rightarrow-e\left(4 \pi|\boldsymbol{x}|^{2}\right)^{-1} \delta(|\boldsymbol{x}|)$, together with the assumption that $\left\|\boldsymbol{w}_{\mathrm{E}}\right\|<C$, the Nodvik charge-current density (5.3) reduces to the familiar expression for the spinless point charge [26, 31],

$$
\boldsymbol{J}_{\text {point charge }}(\boldsymbol{x})=-e \int_{-\infty}^{+\infty} \boldsymbol{u}(\tau) \delta(\boldsymbol{x}-\boldsymbol{q}(\tau)) \mathrm{d} \tau .
$$

\section{Minimal coupling of particle and fields}

In order to obtain the dynamical equations of massive LED, we have to couple the relativistic Maxwell-Lorentz equations for the electromagnetic fields and the relativistic equations for the particle's world-line and gyrograph in a consistent manner. Since both the dynamics of the bare particle and the dynamics of the Maxwell-Lorentz field derive separately from some principle of 'least' action, we can follow Nodvik [15] and apply the standard procedure of minimal coupling to the action functionals and then postulate the principle of least action for this new, joint action. As a general reference for relativistic action principles we recommend [32]. 


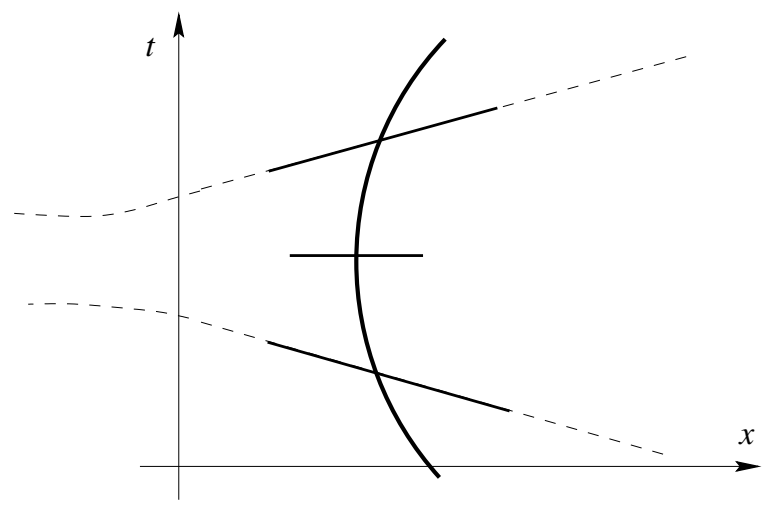

Figure 1: An example of an admissible four-domain $\Xi$, which contains an ordered history of the particle. The extra-bold line is the world-line of the particle. The solid lines indicate the support of $f_{\mathrm{e}}(\|\mathbf{x}-\boldsymbol{q}(\tau)\|)$ restricted to the simultaneity space slices $\boldsymbol{u}(\tau) \cdot(\mathbf{x}-\boldsymbol{q}(\tau))=0$ at $\tau_{1}, \tau_{2}$ and an intermediate value of $\tau$. The dashed lines are the space slices that bound $\Xi$.

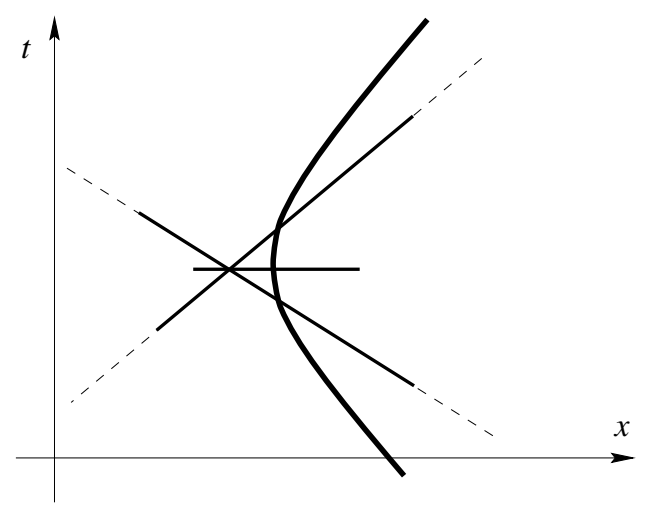

Figure 2: An example of a non-admissible four-domain $\Xi$. No ordered particle history is possible because the supports of the functions $\mathbf{x} \mapsto f_{\mathrm{e}}(\|\mathbf{x}-\boldsymbol{q}(\tau)\|)$ restricted to different space slices of simultaneity $\mathbf{u}(\tau) \cdot(\boldsymbol{x}-\boldsymbol{q}(\tau))=0$ must intersect.

\subsection{The action of massive LED}

The action is defined through an integral over any four-domain $\Xi$ which is sandwiched between two disjoint spacelike three-subspaces that contain the intersections of the supports of $f_{\mathrm{e}}(\|\mathbf{x}-\boldsymbol{q}(\tau)\|)$ (and of $f_{\mathrm{m}}(\|\mathbf{x}-\boldsymbol{q}(\tau)\|)$ ) with the spacelike slices $\mathbf{u}(\tau) \cdot(\mathbf{x}-\boldsymbol{q}(\tau))=0$ of events which are simultaneous to the events at $\boldsymbol{q}(\tau)$ for $\tau=\tau_{1}$ and $\tau=\tau_{2}$, respectively. Such a four-domain is called 'admissible.' It contains ordered particle histories, defined by particle world-lines which connect the supports of $f_{\mathrm{e}}(\|\mathbf{x}-\boldsymbol{q}(\tau)\|)$ in the slices $\mathbf{u}(\tau) \cdot(\mathbf{x}-\boldsymbol{q}(\tau))=0$ at $\tau=\tau_{1}$ and $\tau=\tau_{2}$ in such a way that successive space slices of simultaneity do not intersect in the support of $f_{\mathrm{e}}(\|\mathbf{x}-\boldsymbol{q}(\tau)\|)$; see Figure 1. Not every world-line corresponds to an ordered history. The space slices of simultaneity for two different values of $\tau$ may intersect in the support $f_{\mathrm{e}}(\|\mathbf{x}-\boldsymbol{q}(\tau)\|)$ if the particle acceleration is too strong; see Figure 2.

To formulate our minimally coupled action functional, we also need to introduce the fourvector of the electromagnetic potential. We recall that (5.1) implies that $\mathbf{F}$ is the exterior 
derivative of an electromagnetic potential four-vector $\boldsymbol{A}(\mathbf{x})$, i.e. $\mathbf{F}(\mathbf{x})=\boldsymbol{\nabla}_{\mathrm{g}} \wedge \boldsymbol{A}(\mathbf{x})$. We will work within the class of $\boldsymbol{A}$ that satisfy the so-called Lorentz gauge condition $\boldsymbol{\nabla}_{\mathrm{g}} \cdot \boldsymbol{A}=0$, which still allows the gauge transformation $\boldsymbol{A} \rightarrow \boldsymbol{A}+\boldsymbol{\nabla}_{\mathrm{g}} \psi$ for any $\psi$ satisfying the wave equation $\square \psi=0$.

Given an admissible $\Xi$, the action functional $\mathcal{A}$ is given as integral

$$
\mathcal{A}=\int_{\Xi} \mathcal{L}(\boldsymbol{x}) \mathrm{d}^{4} \mathbf{x}
$$

where $\mathcal{L}(\mathbf{x})$ is the Lagrangian four-density for the minimally coupled field-particle system,

$$
\mathcal{L}=\mathcal{L}_{\text {field }}+\mathcal{L}_{\text {coupl }}+\mathcal{L}_{\text {bare }} .
$$

The first term is Schwarzschild's Lagrangian density for the free electromagnetic field of Maxwell, i.e.

$$
\mathcal{L}_{\text {field }}(\mathbf{x}) \stackrel{\text { def }}{=}-\frac{1}{16 \pi} \operatorname{Tr}(\mathbf{F}(\mathbf{x}) \cdot \mathbf{F}(\mathbf{x})),
$$

and the second term is the standard Lagrangian density for the minimal electromagnetic coupling of particle and field degrees of freedom, i.e.

$$
\mathcal{L}_{\text {coupl }}(\mathbf{x}) \stackrel{\text { def }}{=} \boldsymbol{J}(\mathbf{x}) \cdot \boldsymbol{A}(\mathbf{x}),
$$

where $\boldsymbol{J}$ is the Nodvik charge-current density given in (5.3). The last term is the Lagrangian density for our bare particle,

$$
\mathcal{L}_{\text {bare }}(\mathbf{x}) \stackrel{\text { def }}{=}-\int_{\tau_{1}}^{\tau_{2}} \sqrt{1-\left\|\boldsymbol{\Omega}_{\mathrm{E}} \cdot(\mathbf{x}-\boldsymbol{q})\right\|^{2}} f_{\mathrm{m}}(\|\mathbf{x}-\boldsymbol{q}\|) \delta(\mathbf{u} \cdot(\mathbf{x}-\boldsymbol{q})) \mathrm{d} \tau .
$$

\subsection{Invariants for the action functional}

Our action functional is invariant under several symmetry transformations of base and target spaces, associated with which are invariants that give the physical conservation laws. These invariants are listed below. We consider only ordered histories, so that the domain of integration in the conservation laws can be written $\mathbb{R}^{1,3}$ instead of $\Xi$.

\subsubsection{Invariance under base space transformation}

Invariance of the action functional under translations of spacetime implies conservation of the total Minkowski momentum four-vector $\boldsymbol{P}$ of the field-particle system, the constant time component of which expresses energy conservation, the space components the conservation of linear three-momentum. The Minkowski momentum of the field-particle system can be written in the form

$$
\boldsymbol{P}=\mathbf{M}_{\mathrm{fp}}(\tau) \cdot \mathbf{u}(\tau),
$$


where

$$
\mathbf{M}_{\mathrm{fp}}(\tau)=\mathcal{M}_{\mathrm{b}}\left(\left\|\boldsymbol{w}_{\mathrm{E}}(\tau)\right\|\right) \mathbf{g}+\mathbf{M}_{\mathrm{N}}(\tau)+\int_{\mathbb{R}^{1,3}} \mathbf{T}(\mathbf{x}) \delta(\mathbf{u}(\tau) \cdot(\boldsymbol{x}-\mathbf{q}(\tau))) \mathrm{d}^{4} \mathbf{x}
$$

is the symmetric field-particle tensor mass, itself composed of the following tensor masses: the first term on its r.h.s. is just the bare gyrational mass of the particle written as diagonal tensor with the help of the metric tensor $\mathbf{g}$; the second term on its r.h.s is the symmetric Nodvik tensor mass,

$$
\mathbf{M}_{\mathrm{N}}(\tau)=-\int_{\mathbb{R}^{1,3}}\left[\mathbf{x} \otimes \mathbf{x},\left[\mathbf{F}(\boldsymbol{q}(\tau)+\mathbf{x}), \boldsymbol{\Omega}_{\mathrm{E}}(\tau)\right]_{+}\right]_{+} f_{\mathrm{e}}(\|\boldsymbol{x}\|) \delta(\mathbf{u}(\tau) \cdot \boldsymbol{x}) \mathrm{d}^{4} \boldsymbol{x}
$$

extracted (see Appendix A.1) from the Minkowski momentum four-vector of electromagnetic spin-orbit coupling given in [15]; the last term on its r.h.s. finally is the electromagnetic field mass tensor, with

$$
\mathbf{T}(\mathbf{x})=\frac{1}{4 \pi}\left(\mathbf{F}(\mathbf{x}) \cdot \mathbf{F}(\mathbf{x})-\frac{1}{4} \operatorname{Tr}(\mathbf{F}(\mathbf{x}) \cdot \mathbf{F}(\mathbf{x})) \mathbf{g}\right)
$$

the conventional traceless tensor of the electromagnetic energy-momentum-stress density, a symmetric tensor field of rank two. It's four-divergence, given by

$$
\nabla_{\mathrm{g}} \cdot \mathbf{T}(\mathbf{x})=\mathbf{F}(\mathbf{x}) \cdot \mathbf{J}(\mathbf{x})
$$

does in general not vanish on the support of the particle densities $f_{\mathrm{m}}$ and $f_{\mathrm{e}}$; in a nutshell, this is the origin for the need of the Fermi prescription of integration over space slices of simultaneity (cf. 23, 27]).

Invariance of the action functional under spacetime rotations implies conservation of the anti-symmetric tensor of total Minkowski angular momentum $\mathbf{L}$ of the field-particle system,

$$
\mathbf{L}=\mathbf{S}_{\mathrm{b}}(\tau)+\boldsymbol{q}(\tau) \wedge \boldsymbol{P}+\int_{\mathbb{R}^{1,3}} \mathbf{x} \wedge(\mathbf{T}(\mathbf{x}+\boldsymbol{q}(\tau)) \cdot \mathbf{u}(\tau)) \delta(\mathbf{u}(\tau) \cdot \mathbf{x}) \mathrm{d}^{4} \mathbf{x}
$$

where $\boldsymbol{P}$ and $\mathbf{T}$ are given above, and where

$$
\mathbf{S}_{\mathrm{b}}(\tau)=-\int_{\mathbb{R}^{1,3}} \boldsymbol{x} \wedge \frac{\boldsymbol{\Omega}_{\mathrm{E}}(\tau) \cdot \mathbf{x}}{\sqrt{1-\left\|\Omega_{\mathrm{E}}(\tau) \cdot \boldsymbol{x}\right\|^{2}}} f_{\mathrm{m}}(\|\mathbf{x}\|) \delta(\mathbf{u}(\tau) \cdot \boldsymbol{x}) \mathrm{d}^{4} \mathbf{x}
$$

is the anti-symmetric tensor of bare Minkowski spin (about $\mathbf{q}$ ) of the particle. The tensor $\mathbf{S}_{\mathrm{b}}$ is dual to the bare spin four-vector (4.8), hence it is of space-space type w.r.t. $\mathbf{u}$, viz.

$$
\mathbf{S}_{\mathrm{b}} \cdot \mathbf{u}=0
$$

Since $\mathbf{L}$ is anti-symmetric, its constancy expresses six individual physical conservation laws. The constancy of the three space-space components expresses the conservation of the total angular momentum, and the constancy of the three time-space components correspond to the statement that the center of mass of the field-particle system moves with constant threevelocity along a straight line in the three-space slice of the Lorentz frame. 


\subsubsection{Invariance under target space transformation}

Invariance of the action functional under gauge transformations $\boldsymbol{A} \rightarrow \boldsymbol{A}+\boldsymbol{\nabla}_{\mathrm{g}} \psi$ implies charge conservation locally, i.e. (5.3) satisfies the continuity equation

$$
\nabla_{\mathrm{g}} \cdot \boldsymbol{J}(\mathbf{x})=0
$$

which is demanded by the Maxwell-Lorentz equations and readily verified to hold also by direct computation with (5.3), cf. [15]. Of course, (6.14) implies the conservation of the total charge

$$
Q=-\int_{\mathbb{R}^{1,3}} \boldsymbol{e}_{0} \cdot \boldsymbol{J}(\mathbf{x}) \delta\left(\boldsymbol{e}_{0} \cdot \mathbf{x}-t_{0}\right) \mathrm{d}^{4} \mathbf{x},
$$

i.e. $Q=-e$ in our model of the electron, for all $t_{0}$.

In addition, there is a symmetry associated with the spherical rest symmetry of the particle, giving invariance of the action functional under shift of the Euler angle around the instantaneous axis of gyration. (For the definition of the Euler angles, see Appendix A.1.) This corresponds to the conservation of

$$
s^{2}=-\frac{1}{2} \operatorname{Tr}\left(\mathbf{S}^{\perp}(\tau) \cdot \mathbf{S}^{\perp}(\tau)\right)
$$

where

$$
\mathbf{S}^{\perp}=\mathbf{S}+[\mathbf{u} \otimes \mathbf{u}, \mathbf{S}]_{+}
$$

is the space-space part w.r.t. $\mathbf{u}$ of the anti-symmetric tensor of total Minkowski spin,

$$
\mathbf{S}(\tau) \stackrel{\text { def }}{=} \mathbf{S}_{\mathrm{f}}(\tau)+\mathbf{S}_{\mathrm{b}}(\tau)
$$

with

$$
\mathbf{S}_{\mathrm{f}}(\tau) \stackrel{\text { def }}{=} \int_{\mathbb{R}^{1,3}}(\mathbf{x}-\boldsymbol{q}(\tau)) \wedge \boldsymbol{A}(\mathbf{x}) f_{\mathrm{e}}(\|\mathbf{x}-\boldsymbol{q}(\tau)\|) \delta(\mathbf{u}(\tau) \cdot(\mathbf{x}-\boldsymbol{q}(\tau))) \mathrm{d}^{4} \mathbf{x}
$$

the tensor of the particle's electromagnetic Minkowski spin about $\boldsymbol{q}(\tau)$.

We remark that despite its appearance, $\mathbf{S}_{\mathrm{f}}$ is invariant under a gauge transformation $\boldsymbol{A} \rightarrow \boldsymbol{A}+\boldsymbol{\nabla}_{\mathrm{g}} \psi$.

The law for $s^{2}$ means that the dual four-vector to $\mathbf{S}^{\perp}$ evolves on the spacelike genus-one hyperboloid.

\subsection{The principle of 'least' action}

Our action principle is formulated for a fixed four-domain $\Xi$, with the constraints $\delta \mathbf{u}(\tau)=0$ for $\tau=\tau_{1}$ and $\tau=\tau_{2}$. Likewise, the variations of the world-line $\delta \boldsymbol{q}(\tau)$ and of the Euler angles (see Appendix A.1) vanish at $\tau=\tau_{1}$ and $\tau=\tau_{2}$. Following standard physics terminology we refer to this Hamiltonian principle of stationary action as 'principle of least action,' although we do not investigate the second variation here. 
The particle world-line satisfies the variational principle

$$
\delta\left(\mathcal{A}_{\text {bare }}+\mathcal{A}_{\text {coupl }}\right)=0
$$

under an independent deformation of the world-line $\tau \mapsto \boldsymbol{q}(\tau)$ (with $\tau \in\left[\tau_{1}, \tau_{2}\right]$ ) into a worldline $\tau \mapsto \boldsymbol{q}(\tau)+\delta \boldsymbol{q}(\tau)$ (with $\tau$ in the same range), with Euler variables $\boldsymbol{\theta}$ and four-potential $\boldsymbol{A}$ fixed.

The gyrograph satisfies the variational principle

$$
\delta\left(\mathcal{A}_{\text {bare }}+\mathcal{A}_{\text {coupl }}\right)=0
$$

under an independent variation of the gyrograph of the particle, with fixed world-line $\tau \mapsto \boldsymbol{q}$ and four-potential $\boldsymbol{A}$.

The fields satisfy the standard Schwarzschild principle [18, [16],

$$
\delta\left(\mathcal{A}_{\text {field }}+\mathcal{A}_{\text {coupl }}\right)=0
$$

for prescribed world-line $\tau \mapsto \boldsymbol{q}$ and gyrograph $\tau \mapsto \boldsymbol{\Omega}_{\mathrm{E}}$.

\section{The dynamical equations of massive LED}

The dynamical equations are the Euler-Lagrange equations for the variational principle

$$
\delta \mathcal{A}=0,
$$

with $\boldsymbol{q}, \boldsymbol{A}$ and the Euler angles $\boldsymbol{\theta}$ treated as independent variables. We will state the relativistic dynamical equations in a format closely reminiscent of the semi-relativistic equations of the massive Abraham theory described in Appendix A.3.

\subsection{Field equations}

Recalling that $\mathbf{F}=\boldsymbol{\nabla}_{\mathrm{g}} \wedge \boldsymbol{A}$ by definition, which already implies the homogeneous MaxwellLorentz equations (5.1), the principle of least action together with $\boldsymbol{\nabla}_{\mathrm{g}} \cdot \boldsymbol{A}=0$ now yields

the equivalent potential form of the inhomogeneous Maxwell-Lorentz equations, i.e. the inhomogeneous wave equation

$$
\square \boldsymbol{A}(\mathbf{x})=4 \pi \boldsymbol{J}(\mathbf{x}),
$$

with $\boldsymbol{J}$ Nodvik's four-vector of charge-current density given in (5.3).

\subsection{World-line equations}

The world-line equations read

$$
\frac{\mathrm{d}}{\mathrm{d} \tau} \boldsymbol{p}=\boldsymbol{f},
$$


where

$$
\boldsymbol{p}(\tau)=\mathbf{M}(\tau) \cdot \mathbf{u}(\tau)
$$

is the Minkowski momentum of the particle, with $\mathbf{M}=\mathcal{M}_{\mathrm{b}} \mathbf{g}+\mathbf{M}_{\mathrm{N}}$ (see (6.7)), and where

$$
\boldsymbol{f}(\tau)=\int_{\mathbb{R}^{1,3}} \mathbf{F}(\mathbf{x}) \cdot \boldsymbol{U}(\mathbf{x} ; \tau) f_{\mathrm{e}}(\|\mathbf{x}-\boldsymbol{q}(\tau)\|) \delta(\boldsymbol{u}(\tau) \cdot(\mathbf{x}-\boldsymbol{q}(\tau))) \mathrm{d}^{4} \mathbf{x},
$$

is Nodvik's [15] Abraham-Lorentz type Minkowski force.

\subsection{Gyrograph equations}

The equations for the gyrograph read

$$
\frac{\mathrm{d}}{\mathrm{d} \tau} \mathbf{S}_{\mathrm{b}}+\left[\boldsymbol{\Omega}_{\mathrm{FW}}, \mathbf{S}_{\mathrm{b}}\right]_{-}=\mathbf{t}
$$

where

$$
\mathbf{t}(\tau)=\int_{\mathbb{R}^{1,3}}(\mathbf{x}-\boldsymbol{q}(\tau)) \wedge(\mathbf{F}(\mathbf{x}) \cdot \boldsymbol{U}(\mathbf{x}, \tau))^{\perp} f_{\mathrm{e}}(\|\mathbf{x}-\boldsymbol{q}(\tau)\|) \delta(\mathbf{u}(\tau) \cdot(\mathbf{x}-\boldsymbol{q}(\tau))) \mathrm{d}^{4} \mathbf{x}
$$

is the anti-symmetric tensor of the Abraham-Lorentz type Minkowski torque, with $\mathbf{a}^{\perp} \stackrel{\text { def }}{=}$ $(\mathbf{g}+\mathbf{u} \otimes \mathbf{u}) \cdot \mathbf{a}$ the projection of the four-vector $\mathbf{a}$ onto the space slice w.r.t. $\mathbf{u}$. The space projector $\mathbf{g}+\mathbf{u} \otimes \mathbf{u}$ under the integral guarantees that (7.6) preserves the intrinsic space-space character of $\mathbf{S}_{\mathrm{b}}$. The commutator with the Fermi-Walker tensor accounts for the four-precession of the Fermi-Walker-transported, natural co-moving frame with respect to which the inertial gyrations are defined.

\section{Evolution of the state in massive LED}

In the concise component-free Minkowski space notation the similarity of the world-line equation (7.3), supplemented with (7.4) and (7.5), to the Newtonian type evolution equation (A.84) of the semi-relativistic massive LED with spin is clearly recognizable, and so is the similarity of the gyrograph equation (7.6), supplemented with (7.7), to the Eulerian evolution equation (A.86). The almost familiar appearance of the dynamical equations of our relativistic massive LED is, however, deceptive. As we will now see, the physically natural interpretation of these dynamical equations as a set of evolution equations for the dynamical state of the theory unveils some radically new elements of physical reality (as defined by the theory).

The characteristic of a physical state, loosely speaking, is that its specification at any particular instant completely determines, via first-order evolution equations, the time derivative of the state at that instant and, hence, allows to continue uniquely to the state at the next instant. To find out what precisely constitutes a 'state at any particular instant' in 
our massive LED, we have to analyze for which dynamical variables the equations pose a first-order initial value problem (Cauchy problem) in an orderly manner. This is done in the following two subsections. We then address the problem of nearly free global evolutions. Since the evolution equations of our massive LED are implicit in a rather unfamiliar manner, we first rewrite them into a quasi-explicit format. We then propose a direct generalization of the familiar Picard iteration to solve the equations - in principle. We finally remark on the question of global well-posedness of the Cauchy problem for arbitrary admissible states. This issue is only partially settled. Various possible 'mechanisms' that may cause some sort of singularity to occur at a finite time have to be controlled. However, for the special case of purely gyrational particle dynamics coupled to the Maxwell-Lorentz fields we succeeded in proving, by using our generalized Picard iteration, a global existence and uniqueness result. This proof is going to appear in [33].

\subsection{The states in massive LED}

We first need to clarify the phrase at an instant of time. In special relativity, this is a quite flexible notion. Generally it refers to the collection of all the events on a three-dimensional, simply connected, spacelike hypersurface. We assume without loss of generality that such a hypersurface is defined as the level set $\Sigma_{t}=\{\mathbf{x}: T(\mathbf{x})=t\}$ of a differentiable function $T$ on spacetime. Here, a three-dimensional hypersurface $\Sigma_{t}$ is called spacelike if $\boldsymbol{\nabla}_{\mathrm{g}} T(\mathbf{x})$ is timelike at each $\boldsymbol{x}$ in $\Sigma_{t}$. If the intersection of any two such spacelike hypersurfaces $\Sigma_{t_{1}}$ and $\Sigma_{t_{2}}$, with $t_{1} \neq t_{2}$, is empty, and if furthermore the four-dimensional set $\cup_{t \in \mathbb{R}} \Sigma_{t}$ covers spacetime, $\cup_{t \in \mathbb{R}} \Sigma_{t}$ is called a foliation of spacetime (generated by $T$ ), and each three-dimensional hypersurface $\Sigma_{t}$ is called a leaf of that foliation. For example, the standard foliation of a Lorentz frame $\mathcal{F}_{\mathrm{L}}=\left\{\boldsymbol{e}_{0}, \boldsymbol{e}_{1}, \boldsymbol{e}_{2}, \boldsymbol{e}_{3}\right\}$ into affine space slices of the form $\left\{\mathbf{x}: x^{0}=t\right\}$ is generated by the function $T_{\mathcal{F}_{\mathrm{L}}}(\mathbf{x}) \stackrel{\text { def }}{=}-\boldsymbol{e}_{0} \cdot \mathbf{x}$, which has a constant timelike four-gradient, $\nabla_{\mathrm{g}} T_{\mathcal{F}_{\mathrm{L}}}(\mathbf{x})=-\boldsymbol{e}_{0}$. There are uncountably many foliations of spacetime among which one has to find any one that is compatible with the structure of the dynamical equations, preferably a convenient one. Unfortunately, as we shall see in a moment, the standard foliation is not compatible with the dynamical equations of massive LED. In any event, a 'state at an instant $t$ ' thus means the specification of the complete set of dynamical variables on the leaf with index $t$ of a compatible foliation of spacetime.

To find out what constitutes such a complete set of dynamical variables, we notice that our dynamical equations are of first order in the proper-time derivative of each of the kinematical particle variables $\boldsymbol{q}, \boldsymbol{u}, \Omega_{\mathrm{E}}$, and of first order in the Lorentz time derivative of the fields F. A dynamical state in our massive LED will therefore be a set of the form $\left\{\boldsymbol{q}(\tau), \boldsymbol{u}(\tau), \boldsymbol{\Omega}_{\mathrm{E}}(\tau), \mathbf{F}().\right\}$, specified on a leaf $\Sigma_{t}$ of a compatible foliation of spacetime indexed by $t$, satisfying various consistency conditions that constrain the values of $\boldsymbol{q}, \mathbf{u}, \boldsymbol{\Omega}_{\mathrm{E}}$ at $\tau$ and the field $\mathbf{F}\left(\right.$. ) on the leaf. In particular, the four-velocity has to satisfy $\|\mathbf{u}\|^{2}=-1$, and the anti-symmetric rank-two Euler tensor has to satisfy $\Omega_{\mathrm{E}}(\tau) \cdot \boldsymbol{u}(\tau)=\boldsymbol{0}$. Furthermore, the Maxwell-Lorentz equations impose the constraint that $\mathbf{F}$ satisfy the three-divergence part of the Maxwell-Lorentz equations, which fixes the longitudinal part of $\mathbf{F}$ as a passive attribute of the charged particle.

These are all local constraints imposed by relativity, which occur also in the traditional 
limiting theories, i.e. the relativistic point charge mechanics in given external force fields, the mechanics of a point spin in given external torque fields, and the Maxwell electrodynamics with given source terms, in all of which cases the constraints are known to propagate in time if they are satisfied initially. This will be true also for our massive Lorentz electrodynamics.

However, our covariant first-order dynamical equations are also mildly non-local, and this is going to be reflected in the compatibility conditions to be satisfied by the foliation of spacetime. Namely, since the first-order proper-time derivatives of $\boldsymbol{u}$ and $\boldsymbol{\Omega}_{\mathrm{E}}$ at propertime $\tau$ are given (implicitly) in terms of integrals of the fields over the particle's support in its instantaneous space slice of simultaneity, the transversal part (i.e. the dynamical degrees of freedom) of $\mathbf{F}(\mathbf{x})$ must be known for all $\mathbf{x} \in \operatorname{supp}\left(f_{\mathrm{e}}(\|\cdot\|)\right)$ satisfying $\mathbf{u}(\tau) \cdot(\mathbf{x}-$ $\boldsymbol{q}(\tau))=0$ (recall that the passive longitudinal part of the field $\mathbf{F}(\mathbf{x})$ is not a dynamical degree of freedom). Hence, we say that a foliation of spacetime is compatible with a worldline $\tau \mapsto \boldsymbol{q}(\tau)$ of an extended Lorentz electron (i.e., a particle history) if each leaf of the foliation contains precisely one of the sets $\{\boldsymbol{u}(\tau) \cdot(\mathbf{x}-\boldsymbol{q}(\tau))=0\} \cap \operatorname{supp}\left(f_{\mathrm{e}}(\|\cdot\|)\right)$ (for simplicity, we assume that $\left.\operatorname{supp}\left(f_{\mathrm{m}}\right)=\operatorname{supp}\left(f_{\mathrm{e}}\right)\right)$. A compatible foliation sets the spacetime parameterization w.r.t. which the Maxwell-Lorentz equations must be solved in order to enable the evaluation of the Minkowski force and torque.

A compatible foliation is of course not known explicitly until the particle world-line is known; it has to be determined along with the particle world-line and the fields by solving the second variation equations for the extrinsic curvature of the leaf $\Sigma_{t}$ coupled with the constraint equations of Gauss-Codacci and Gauss in the complement of the sets $\mathbf{x} \in \operatorname{supp}\left(f_{\mathrm{e}}(\|\cdot\|)\right)$ satisfying $\mathbf{u}(\tau) \cdot(\mathbf{x}-\boldsymbol{q}(\tau))=0$, which is a non-linear free boundary problem. Interestingly, these circumstances inject technical elements from general relativity into this special relativistic theory.

Remark: No foliation is compatible with a particle history that is not ordered in the sense described earlier (see Fig.1 and 2).

The condition that the particle history be ordered imposes size restrictions on the accelerating fields. Indeed, we easily find locally consistent data for which the particle's threeacceleration in its instantaneous Lorentz rest frame exceeds the value $1 / R$, where $R$ is the radius of the particle. For such accelerations, part of the support of the particle in its instantaneous space slice of simultaneity will be launched backward in Lorentz time rather than forward, and this motion does not generate an ordered history. This implies the following bound on the particle's initial three-acceleration in the Lorentz frame in which the particle is instantaneously at rest,

$$
|\ddot{\boldsymbol{q}}| R<1
$$

which has to be translated into bounds on the accelerating field strengths in the instantaneous particle rest frame. We will soon see that these bounds on the field strengths are about 100 times the particle's self-fields at it's surface which, happily, is way outside the physically reasonable range of data for a classical electrodynamics. Last not least, the condition that the gyrational speed for points on the particle's equator must be subluminal imposes a size restriction on $\Omega_{\mathrm{E}}$, viz. (recall that $c=1$ )

$$
\left\|\mathbf{w}_{\mathrm{E}}\right\| R<1 .
$$




\subsection{The Cauchy problem for the state}

We now formulate the initial value problem for the states. We begin by specifying the generic initial data on a convenient initial leaf. The compatible foliation itself is characterized subsequently by dynamical equations which together with the equations of massive LED (rewritten w.r.t. the compatible foliation) pose a nonlinear, implicit Cauchy problem for the joint evolution of the state and the leaf of the foliation on which the state is defined.

\subsubsection{Cauchy data and the initial leaf}

Since by a suitable spacetime translation we can always transform to a Lorentz frame in which the event of the particle's initial spacetime position is at the origin of that Lorentz frame, and since by a suitable Lorentz boost we can furthermore transform to another Lorentz frame with the same origin but in which the particle's initial three-velocity vanishes, and since finally we can choose the proper-time of the particle equal to zero at that initial event, the Cauchy data for the world-line $\tau \mapsto \boldsymbol{q}(\tau)$ can be chosen conveniently as

$$
\begin{aligned}
& \boldsymbol{q}(0)=\boldsymbol{0} \\
& \mathbf{u}(0)=\boldsymbol{e}_{0} .
\end{aligned}
$$

Notice that $\boldsymbol{e}_{0}$ satisfies the constraint $\left\|\boldsymbol{e}_{0}\right\|^{2}=-1$ required of a four-velocity. Moreover, by at most a space rotation of this Lorentz frame we can achieve that $\boldsymbol{w}_{\mathrm{E}}(0)$, the dual vector to $\boldsymbol{\Omega}_{\mathrm{E}}(0)$, has only a single non-vanishing component, say the 3 component, $\boldsymbol{w}_{\mathrm{E}}(0)=\omega_{\mathrm{E} 0} \boldsymbol{e}_{3}$, with $\omega_{\mathrm{E} 0}$ satisfying (8.2) but otherwise at our disposal. The Cauchy data for the particle's gyrograph $\tau \mapsto \Omega_{\mathrm{E}}(\tau)$ are then of the form

$$
\boldsymbol{\Omega}_{\mathrm{E}}(0)=\omega_{\mathrm{E} 0} \boldsymbol{e}_{1} \wedge \boldsymbol{e}_{2} .
$$

Clearly, $\boldsymbol{\Omega}_{\mathrm{E}}(0)$ is anti-symmetric, and since $\left(\boldsymbol{e}_{1} \wedge \boldsymbol{e}_{2}\right) \cdot \boldsymbol{e}_{0}=\boldsymbol{O}$, also of space-space type w.r.t. $\mathbf{u}(0)$.

The Lorentz frame in which (8.3), (8.4), and (8.5) holds will be referred to as the initial rest frame $\mathcal{F}_{\mathrm{L} 0}$. The initial leaf $\Sigma_{0}$ of all of our foliations is now chosen to be the particle's affine space slice of simultaneity in the initial rest frame $\mathcal{F}_{\mathrm{L} 0}$, i.e. the set $\left\{\boldsymbol{e}_{0} \cdot \boldsymbol{X}=0\right\}$ on which the Lorentz time $t\left(=x^{0}\right)=0$.

Accordingly, the Cauchy data for $\mathbf{F}(\mathbf{x})$ are specified on $\Sigma_{0}$,

$$
\left.\mathbf{F}(\boldsymbol{x})\right|_{t=0}=\mathbf{F}_{0}(\boldsymbol{x}),
$$

where $\mathbf{F}_{0}(\boldsymbol{x})$ satisfies the three-divergence equations in the Maxwell-Lorentz equations restricted to $\Sigma_{0}$. To make this somewhat implicit characterization of $\mathbf{F}_{0}$ more explicit, we decompose $\mathbf{F}_{0}$ into its electric and magnetic components w.r.t. $\mathcal{F}_{\mathrm{L} 0}$ and group them together into a complex electromagnetic three-vector field on $\Sigma_{0}$,

$$
\boldsymbol{\mathcal { G }}_{0} \stackrel{\text { def }}{=} \mathcal{E}_{0}+i \boldsymbol{\mathcal { B }}_{0},
$$

whose real and imaginary part are, respectively, the electric (i.e. time-space) and magnetic (i.e. space-space) components of the field tensor $\mathbf{F}_{0}$ in $\Sigma_{0}$. The three-divergence equations 
in $\Sigma_{0}$ now read

$$
\boldsymbol{\nabla} \cdot \mathcal{G}_{0}(\boldsymbol{x})=4 \pi \rho_{0}(\boldsymbol{x})
$$

where $\rho_{0}$ is the time component of $\boldsymbol{J}(0)$, uniquely determined in terms of the initial values of the other state variables $\boldsymbol{q}_{0}, \boldsymbol{u}_{0}$ and $\boldsymbol{\Omega}_{\mathrm{E} 0}$.

Remark: Through the presence of $\stackrel{\circ}{\mathbf{u}}$ in the right-hand side of (5.3) the (initial) constraint appears to be implicit. However, by direct computation it is readily verified that the $\stackrel{\circ}{\mathbf{u}}$ contribution to $\boldsymbol{J}$ drops out initially as a consequence of the sphericity of the charge distribution in the particle's instantaneous rest frame, which initially coincides with the Lorentz frame $\mathcal{F}_{\mathrm{L} 0}$.

The initial fields are chosen in accordance with the asymptotic condition that $\mathcal{G}_{0}(\boldsymbol{x}) \rightarrow \mathbf{0}$ as $|\boldsymbol{x}| \rightarrow \infty$, the real part as $\mathcal{E}_{0}(\boldsymbol{x}) \sim-e \boldsymbol{x} /|\boldsymbol{x}|^{3}+o\left(|\boldsymbol{x}|^{-2}\right)$, the imaginary part sufficiently fast for surface integrals at infinity involving $\mathcal{B}_{0}$ to vanish.

\subsubsection{Evolution equations for state and compatible foliation}

To each differentiable particle world-line starting with (8.3) and (8.4) and generating an ordered particle history (referred to by an index ${ }_{h}$ ), we have to associate a convenient, compatible foliation of spacetime which contains $\Sigma_{0}$ as initial leaf. Unfortunately, the standard foliation is generally not a compatible foliation, for notice that any world-line is the soul of the tubular set of events $\cup_{\tau \geqslant 0} \operatorname{supp}\left(f_{\mathrm{e}}(\|\boldsymbol{x}-\boldsymbol{q}(\tau)\|)\right)$, called the future world-tube of the particle, inside of which the foliation is necessarily given by the $\tau$-indexed affine segments $\{\boldsymbol{x} \mid \mathbf{u}(\tau) \cdot(\mathbf{x}-\boldsymbol{q}(\tau))=0\}$ which intersect with the standard leaf of Lorentz time $t$ but are generally strict subsets of that leaf. However, one expects that it is possible to select a compatible foliation whose leafs, sufficiently far away from the world-line, will arbitrarily closely approximate the leafs of the standard foliation of the initial Lorentz frame, i.e. $\Sigma_{0}$ parallelly transported in Lorentz time. By further choosing the $t$-parameterization of the compatible foliation to be Lorentz time at spatial infinity, by selecting the corresponding value of $\tau$ through the identification $q^{0}(\tau)=t$, and by parameterizing points in $\Sigma_{t}$ by the points $\boldsymbol{x} \in \Sigma_{0}$ through following the integral curves of $\boldsymbol{\nabla}_{\mathrm{g}} T_{h}$, we obtain a foliation which nearly coincides with the standard foliation in most of spacetime, except for a small "wiggle" accompanying the world-line of the particle. Notice that the world-line is one such integral curve, so that in this parameterization the segment of the world-tube that belongs to $\Sigma_{t}$ is always centered at the origin of $\Sigma_{0}$. Equipped with such a global parameterization of a compatible foliation, spacetime is diffeomorphic to the product manifold $\mathbb{R} \times \Sigma_{0}$, i.e. $\mathbb{R} \times \mathbb{R}^{3}$, with spacetime metric

$$
\mathrm{d} s^{2}=-\psi^{2}(\boldsymbol{x}, t) \mathrm{d} t^{2}+\sum_{1 \leqslant k, l \leqslant 3} \gamma^{k l}(\boldsymbol{x}, t) \mathrm{d} x^{k} \mathrm{~d} x^{l}
$$

where $\psi(\boldsymbol{x}, t)=\left\|-\nabla_{\mathrm{g}} T_{h}(\mathbf{x})\right\|^{-1}$ is the lapse function, and the $\gamma^{k l}(\boldsymbol{x}, t)$ are the components of the first fundamental form $\gamma$ of the foliation induced on $\Sigma_{t}$ from the Minkowski metric.

Such an extension of the family of affine segments of the future world-tube to the outside of the world-tube is found by solving the structure equations for a foliation of Minkowski 
spacetime, supplemented with standard foliation asymptotics at spatial infinity, and with appropriate boundary conditions along the perimeter of each segment in the tube which guarantee a differentiable transition at the segment's boundary. The boundary is determined by $\boldsymbol{q}(\tau)$ and $\boldsymbol{u}(\tau)$ (given $f_{\mathrm{e}}$, which as a model parameter does not vary during the dynamics). Since we have to determine the world-line along with the foliation outside the world-tube, our problem belongs to the category of the dynamical free-boundary problems. Notice that even if the world-line is analytic, the foliation is surely not analytic unless the particle is stationary, in which case one can choose the standard foliation of spacetime.

By following [34, 35], we conclude that outside the world-tube the foliation is a solution of the system of evolution equations

$$
\begin{aligned}
& \partial_{t} \gamma=-2 \psi K \\
& \partial_{t} K=-\nabla_{\gamma} \otimes \nabla_{\gamma} \psi+\psi(R i c+K \operatorname{Tr} K-2 K \cdot K)
\end{aligned}
$$

where $K$ is the second fundamental form of the leaf of the foliation, i.e. the extrinsic curvature (actually defined by (8.10) if $T_{h}$ were known), Ric is the Ricci tensor of $\Sigma_{t}$, and $\boldsymbol{\nabla}_{\gamma}$ denotes covariant gradient w.r.t. $\gamma$. Equation (8.11) is known as the second variation formula. In addition, $\gamma, \psi$ and $K$ have to satisfy the constraints given by the Gauss-Codacci equation

$$
\nabla_{\gamma} \cdot K-\nabla_{\gamma} \operatorname{Tr} K=0
$$

and the Gauss equation

$$
\text { Ric }-K \cdot K+K \operatorname{Tr} K=0
$$

which propagate in time if satisfied on the initial leaf. This set of equations has to be supplemented by boundary conditions along the perimeter of the world-tube segment for $\psi$ and for $K$; and by asymptotic conditions as $|\boldsymbol{x}| \rightarrow \infty$. We demand that the foliation be normal, i.e. that $\psi(\boldsymbol{x}, t) \rightarrow 1$ as $|\boldsymbol{x}| \rightarrow \infty$, which means that at spatial infinity the foliation tends to the standard foliation of spacetime. Furthermore, a "gauge" condition is at our disposal, for instance the maximality condition

$$
\operatorname{Tr} K=0 .
$$

The Maxwell-Lorentz equations for the field tensor $\mathbf{F}$ have to be rewritten w.r.t. the foliation in terms of the associated decomposition of $\mathbf{F}$ into electric and magnetic components (cf. [36]), here conveniently grouped together as a complex electromagnetic three-vector field,

$$
\mathcal{G} \stackrel{\text { def }}{=} \mathcal{E}+i \mathcal{B}
$$

the initial decomposition being defined already earlier. The Maxwell-Lorentz evolution equations for $\mathcal{G}$ then read 36

$$
\partial_{t} \mathcal{G}(\boldsymbol{x}, t)=-i \boldsymbol{\nabla}_{\gamma} \times(\psi \mathcal{G})(\boldsymbol{x}, t)-4 \pi(\psi \boldsymbol{j})(\boldsymbol{x}, t)
$$


where $\partial_{t}$ means first-order foliation time derivative (which equals Lorentz time derivative far away from the particle), $\nabla_{\gamma} \times$ is the curl w.r.t. $\gamma^{k, l}$, and the electrical current density threevector $\boldsymbol{j}$ is the $\Sigma_{t}$ projection of $\boldsymbol{J}$. Clearly, (8.16) is an explicit equation for the foliation time derivative of $\mathcal{G}$; however, note that $\boldsymbol{j}(\boldsymbol{x}, t)$ is a (vector) functional not only of all the other state variables $\boldsymbol{\Omega}_{\mathrm{E}}, \boldsymbol{q}, \boldsymbol{u}$ but in addition also of $\stackrel{\circ}{\mathbf{u}}$.

Beside the evolution equation (8.16), $\mathcal{G}$ has to satisfy the three-divergence equations

$$
\boldsymbol{\nabla}_{\gamma} \cdot \mathcal{G}(\boldsymbol{x}, t)=4 \pi \rho(\boldsymbol{x}, t),
$$

where $\rho$, the time component of $\boldsymbol{J}$, is likewise a functional of all the other state variables $\boldsymbol{\Omega}_{\mathrm{E}}, \boldsymbol{q}, \mathbf{u}$ and in addition also of $\stackrel{\circ}{\boldsymbol{u}}$. However, (8.17) is already included in our set of conditions to be satisfied by a state, for notice that by taking the (three-)divergence of (8.16) it follows that a solution $\mathcal{G}(\boldsymbol{x}, t)$ of (8.16) for $\boldsymbol{J}(\mathbf{x})$ given in terms of consistent $\boldsymbol{q}(\tau)$ and $\boldsymbol{\Omega}_{\mathrm{E}}(\tau)$, automatically satisfies (8.17) for all $t>0$ if it satisfies (8.17) initially.

The Maxwell-Lorentz equations are supplemented by the asymptotic condition that $\mathcal{G}(\boldsymbol{x}) \rightarrow \mathbf{0}$ as $|\boldsymbol{x}| \rightarrow \infty$, the real part as $\mathcal{E}(\boldsymbol{x}) \sim-e \boldsymbol{x} /|\boldsymbol{x}|^{3}+o\left(|\boldsymbol{x}|^{-2}\right)$, the imaginary part sufficiently fast for surface integrals at infinity involving $\mathcal{B}$ to vanish.

Finally, following [36] one converts $\mathcal{G}(\boldsymbol{x}, t)$ into $\mathbf{F}$ on a given leaf, which inserted into the world-line and gyrograph equations (7.3) and (7.6) closes the system of evolution equations for the state in massive LED.

In summary, the Cauchy problem for the state evolution turns out to bear a surprising resemblance to the general relativistic evolution problem of a black hole interacting with electromagnetic radiation as described in [36], and to the evolution problem of the vacuum Einstein equations [35]. This gives the equations of massive LED an unexpected additional appeal as a somewhat simpler "toy problem" for these more formidable type of problems of general relativity. At the same time, the enormous progress recently made in the rigorous treatment of the Einstein equations [35] gives hope that the complete control of the Cauchy problem for the state in massive LED can be achieved along similar lines of analysis.

We shall have recourse to the notion of the state in massive LED in our section about scattering. In particular, the invariants stated earlier have to be converted into conservation laws for the evolution w.r.t. a compatible foliation. In the remainder of this section now we will discuss an alternate, global way of looking at massive LED, including an algorithm for the actual computation of solutions to any degree of precision.

\subsection{Nearly free global evolutions}

If, instead of evolving the state locally in time, we look at the putatively harder problem of global particle histories and field decorations of spacetime, one gains however the advantage that one can work with the standard foliation. The price to pay, of having to solve the global problem, will be somewhat lessened by noting that for all practical purposes of computation, one will need only an approximate solution. We restrict ourselves to nearly free global future histories and decorations, with data prescribed at $t=\tau=0$ on $\Sigma_{0}$, as before.

Nearly free evolutions start from Cauchy data which are sufficiently close to a stationary state. For such evolutions, the system of fully implicit massive LED evolution equations can 
be converted into an equivalent, regular quasi-explicit first-order system. We then explain that this quasi-explicit system of equations can be treated via a generalized Picard iteration.円

\subsubsection{Maxwell-Lorentz equations revisited}

We now work with the standard foliation, so that (8.15) is the decomposition of $\mathbf{F}$ into electric and magnetic components w.r.t. this standard foliation. The Maxwell-Lorentz evolution equations for $\mathcal{G}$ now read simply as

$$
\partial_{t} \mathcal{G}(\boldsymbol{x}, t)=-i \boldsymbol{\nabla} \times \boldsymbol{G}(\boldsymbol{x}, t)-4 \pi \boldsymbol{j}(\boldsymbol{x}, t),
$$

where $\partial_{t}$ means first-order Lorentz time derivative, $\nabla \times$ is the conventional curl, and the electrical current density three-vector $\boldsymbol{j}$ is the space component of $\boldsymbol{J}$. Clearly, (8.18) is an explicit equation for the Lorentz time derivative of $\mathcal{G}$. Once again, $\boldsymbol{j}(\boldsymbol{x}, t)$ is a (vector) functional not only of all the other state variables $\boldsymbol{\Omega}_{\mathrm{E}}, \boldsymbol{q}, \mathbf{u}$ but in addition also of $\stackrel{\circ}{\mathbf{u}}$.

Beside the evolution equation (8.18), $\mathcal{G}$ has to satisfy the three-divergence equations

$$
\boldsymbol{\nabla} \cdot \boldsymbol{G}(\boldsymbol{x}, t)=4 \pi \rho(\boldsymbol{x}, t)
$$

where $\rho$, the time component of $\boldsymbol{J}$, is likewise a functional of all the other state variables $\boldsymbol{\Omega}_{\mathrm{E}}, \boldsymbol{q}, \mathbf{u}$ and in addition also of $\stackrel{\circ}{\boldsymbol{u}}$. As explained earlier, (8.19) will be satisfied if it is satisfied by a initial state.

The Maxwell-Lorentz equations are supplemented by the asymptotic condition that $\mathcal{G}(\boldsymbol{x}) \rightarrow \mathbf{0}$ as $|\boldsymbol{x}| \rightarrow \infty$, the real part as $\mathcal{E}(\boldsymbol{x}) \sim-e \boldsymbol{x} /|\boldsymbol{x}|^{3}+o\left(|\boldsymbol{x}|^{-2}\right)$, the imaginary part sufficiently fast for surface integrals at infinity involving $\mathcal{B}$ to vanish.

\subsubsection{Gyrograph equations revisited}

The gyrograph equation (7.6), when viewed as an equation for $\boldsymbol{\Omega}_{\mathrm{E}}$, does not have the desired format in the first-order proper-time derivative of $\Omega_{\mathrm{E}}$ because $\mathbf{S}_{\mathrm{b}}^{\circ}$ involves $\Omega_{\mathrm{E}}$ in a rather complicated manner. However, it is possible and also convenient to view (7.6) as an equation for $\mathbf{S}_{\mathrm{b}}$. To see that $(7.6)$ can indeed be viewed as an equation for $\mathbf{S}_{\mathrm{b}}$, we recall that we required $f_{\mathrm{m}}$ to generate a strictly positive moment of inertia $\mathcal{I}_{\mathrm{b}}$. As a consequence, the map $\left\|\boldsymbol{w}_{\mathrm{E}}\right\| \mapsto \mathcal{I}_{\mathrm{b}}\left(\left\|\boldsymbol{w}_{\mathrm{E}}\right\|\right)$ is strictly positive, increasing, and strictly convex for $\left\|\boldsymbol{w}_{\mathrm{E}}\right\| \in[0,1 / R)$. This implies that we can invert $\mathbf{S}_{\mathrm{b}}=\mathcal{I}_{\mathrm{b}}\left(\left\|\boldsymbol{w}_{\mathrm{E}}\right\|\right) \boldsymbol{\Omega}_{\mathrm{E}}$ to get the Euler angular velocity tensor $\boldsymbol{\Omega}_{\mathrm{E}}$ uniquely in terms of the bare spin tensor $\mathbf{S}_{\mathrm{b}}$, viz. $\boldsymbol{\Omega}_{\mathrm{E}}=\mathcal{I}_{\mathrm{b}}^{-1^{*}}\left(\left\|\mathbf{S}_{\mathrm{b}}\right\|\right) \mathbf{S}_{\mathrm{b}}$, where $\mathcal{I}_{\mathrm{b}}^{-1^{*}}(\xi)=$ $\xi^{-1}\left(\mathcal{I}_{\mathrm{b}} \mathrm{id}\right)^{-1}(\xi)$. With $\boldsymbol{\Omega}_{\mathrm{E}}$ understood in this way as unique (vector) functional of $\mathbf{S}_{\mathrm{b}}$, the

${ }^{1}$ The terminology of "explicit, quasi-explicit, and fully implicit first-order system of equations," is readily explained at hand of just two coupled dynamical variables $\xi(t), \zeta(t)$. (A) a pair of equations $\dot{\xi}=f_{1}(\xi, \zeta)$, $\dot{\zeta}=f_{2}(\xi, \zeta)$ is called an explicit first-order system. (B) a pair $\dot{\xi}=g_{1}(\xi, \zeta, \dot{\zeta}), \dot{\zeta}=g_{2}(\xi, \dot{\xi}, \zeta)$ which is not also of the form (A) is called a regular quasi-explicit first-order system. Thus, in a regular quasi-explicit system each dynamical variable satisfies an explicit equation conditioned on the histories of the other variables. (C) a pair $F_{1}(\xi, \dot{\xi}, \zeta, \dot{\zeta})=0, F_{2}(\xi, \dot{\xi}, \zeta, \dot{\zeta})=0$ which is not also of the form (B) or (A) is called a fully implicit first-order system. The class of fully implicit systems contains the singular quasi-explicit systems, which are of the form $h_{1}(\xi, \zeta, \dot{\zeta}) \dot{\xi}=g_{1}(\xi, \zeta, \dot{\zeta}), h_{2}(\xi, \dot{\xi}, \zeta) \dot{\zeta}=g_{2}(\xi, \dot{\xi}, \zeta)$ with coefficient functions $h_{1}$ and $h_{2}$ that vanish somewhere in the range of their arguments. 
Minkowski torque $\mathbf{t}$ given in (7.7) is now a (tensor) functional of $\mathbf{S}_{\mathrm{b}}, \mathbf{F}, \boldsymbol{q}, \mathbf{u}, \stackrel{\circ}{\mathbf{u}}$, so that (7.6), as equation for $\mathbf{S}_{\mathrm{b}}$,

$$
\frac{\mathrm{d}}{\mathrm{d} \tau} \mathbf{S}_{\mathrm{b}}=-\left[\boldsymbol{\Omega}_{\mathrm{FW}}, \mathbf{S}_{\mathrm{b}}\right]_{-}+\mathbf{t}
$$

is already in the desired format regarding $\mathbf{S}_{\mathrm{b}}$. Notice that not only $\mathbf{t}$ but also $\boldsymbol{\Omega}_{\mathrm{FW}}$ still depends on $\stackrel{\circ}{\boldsymbol{u}}$.

\subsubsection{World-line equations revisited}

We now come to the world-line equation (7.3), which is of second order in the propertime derivatives of $\boldsymbol{q}$. Since we can treat $\boldsymbol{q}$ and $\boldsymbol{u}$ as independent degrees of freedom, this second-order equation is equivalent to two first-order equations; namely, (7.3), now viewed as equation for $\boldsymbol{u}$, together with

$$
\frac{\mathrm{d} \boldsymbol{q}}{\mathrm{d} \tau}=\mathbf{u}
$$

for $\boldsymbol{q}$. While (8.21) already is in the desired format, (7.3) is not. In fact, $\stackrel{\circ}{\mathbf{u}}$ enters through $\stackrel{\circ}{\boldsymbol{p}}$ in the l.h.s. of $(7.3)$ and through the tensor $\Omega_{\mathrm{FW}}=\stackrel{\circ}{\mathbf{u}} \wedge \boldsymbol{u}$ in the r.h.s. of (7.3), so that (7.3) is fully implicit first-order no matter whether viewed as equation for $\boldsymbol{u}$ or for $\boldsymbol{p}$. Switching to $\boldsymbol{p}$ as dynamical variable therefore does not seem to offer an advantage in the conversion of (7.3) into the desired format.

Continuing thus to handle (7.3) as an equation for $\mathbf{u}$, we first sort out all terms involving $\stackrel{\circ}{\mathbf{u}}$. Beginning with the r.h.s. of the world-line equation $(7.3)$, we notice that $\boldsymbol{f}$ can be decomposed into a sum of two terms, one of which is independent of $\stackrel{\circ}{\mathbf{u}}$ and the other one linear in $\stackrel{\circ}{\mathbf{u}}$,

$$
\begin{array}{rl}
\boldsymbol{f}=\int_{\mathbb{R}^{1,3}} & \mathbf{F}(\boldsymbol{q}+\mathbf{x}) \cdot\left(\mathbf{u}-\boldsymbol{\Omega}_{\mathrm{E}} \cdot \mathbf{x}\right) f_{\mathrm{e}}(\|\mathbf{x}\|) \delta(\mathbf{u} \cdot \mathbf{x}) \mathrm{d}^{4} \mathbf{x} \\
& +\int_{\mathbb{R}^{1,3}} \mathbf{F}(\boldsymbol{q}+\mathbf{x}) \cdot \mathbf{u}(\stackrel{\circ}{\mathbf{u}} \cdot \mathbf{x}) f_{\mathrm{e}}(\|\mathbf{x}\|) \delta(\boldsymbol{u} \cdot \mathbf{x}) \mathrm{d}^{4} \mathbf{x}
\end{array}
$$

The l.h.s. of $(7.3)$ can be decomposed in a similar fashion. For $\boldsymbol{p}=\mathbf{M} \cdot \mathbf{u}$ we have

$$
\stackrel{\circ}{\boldsymbol{p}}=\stackrel{\circ}{\mathrm{M}} \cdot \mathbf{u}+\mathbf{M} \cdot \stackrel{\circ}{\mathbf{u}}
$$

with $\mathbf{M}$ given in (4.2), and with

$$
\begin{aligned}
& \stackrel{\circ}{\mathbf{M}}=\stackrel{\circ}{\mathcal{M}}_{\mathrm{b}} \mathbf{g}-\int_{\mathbb{R}^{1,3}}\left[\mathbf{x} \otimes \mathbf{x},\left[\mathbf{F}(\boldsymbol{q}+\mathbf{x}),{\stackrel{\circ}{\boldsymbol{\Omega}_{\mathrm{E}}}}_{+}\right]_{+} f_{\mathrm{e}}(\|\mathbf{x}\|) \delta(\boldsymbol{u} \cdot \mathbf{x}) \mathrm{d}^{4} \boldsymbol{x}\right. \\
& -\int_{\mathbb{R}^{1,3}}\left[\mathbf{x} \otimes \mathbf{x},\left[\boldsymbol{u} \cdot \nabla_{\mathrm{g}} \mathbf{F}(\boldsymbol{q}+\mathbf{x}), \boldsymbol{\Omega}_{\mathrm{E}}\right]_{+}\right]_{+} f_{\mathrm{e}}(\|\mathbf{x}\|) \delta(\boldsymbol{u} \cdot \mathbf{x}) \mathrm{d}^{4} \mathbf{x} \\
& +\int_{\mathbb{R}^{1,3}}\left[\boldsymbol{x} \otimes \mathbf{x},\left[\mathbf{F}(\boldsymbol{q}+\mathbf{x}), \Omega_{\mathrm{E}}\right]_{+}\right]_{+} f_{\mathrm{e}}(\|\mathbf{x}\|)(\stackrel{\circ}{\mathbf{u}} \cdot \mathbf{x}) \mathbf{u} \cdot \nabla_{\mathrm{g}} \delta(\boldsymbol{u} \cdot \mathbf{x}) \mathrm{d}^{4} \boldsymbol{x} .
\end{aligned}
$$


The tensor $\mathbf{M}$ is independent of $\stackrel{\circ}{\mathbf{u}}$, and so are the first three terms of $\stackrel{\circ}{\mathbf{M}}$, while the last term in $\stackrel{\circ}{\mathbf{M}}$ is linear in $\stackrel{\circ}{\mathbf{u}}$. In conclusion, the world-line equation can therefore be written as

$$
\widetilde{\mathbf{M}} \cdot \stackrel{\circ}{\mathbf{u}}=\widetilde{\boldsymbol{f}}
$$

where the four-force $\widetilde{\boldsymbol{f}}$ depends only on $\boldsymbol{q}, \boldsymbol{u}, \boldsymbol{\Omega}_{\mathrm{E}}, \Omega_{\mathrm{E}}, \mathbf{F}$, and $\partial_{t} \mathbf{F}$, thus

$$
\begin{aligned}
& \widetilde{\boldsymbol{f}}=-\stackrel{\circ}{\mathcal{M}}_{\mathrm{b}} \boldsymbol{u}+\int_{\mathbb{R}^{1,3}} \mathbf{F}(\boldsymbol{q}+\mathbf{x}) \cdot\left(\boldsymbol{u}-\boldsymbol{\Omega}_{\mathrm{E}} \cdot \mathbf{x}\right) f_{\mathrm{e}}(\|\boldsymbol{x}\|) \delta(\boldsymbol{u} \cdot \boldsymbol{x}) \mathrm{d}^{4} \boldsymbol{x} \\
& +\int_{\mathbb{R}^{1,3}} \mathbf{x}\left(\mathbf{x} \cdot \Omega_{\mathrm{E}} \cdot\left(\mathbf{u} \cdot \nabla_{\mathrm{g}} \mathbf{F}(\boldsymbol{q}+\mathbf{x})\right) \cdot \mathbf{u}\right) f_{\mathrm{e}}(\|\mathbf{x}\|) \delta(\mathbf{u} \cdot \mathbf{x}) \mathrm{d}^{4} \mathbf{x} \\
& +\int_{\mathbb{R}^{1,3}} \mathbf{x}\left(\mathbf{x} \cdot\left[\mathbf{F}(\boldsymbol{q}+\mathbf{x}), \stackrel{\circ}{\boldsymbol{\Omega}_{\mathrm{E}}}\right]_{+} \cdot \boldsymbol{u}\right) f_{\mathrm{e}}(\|\mathbf{x}\|) \delta(\mathbf{u} \cdot \mathbf{x}) \mathrm{d}^{4} \mathbf{x},
\end{aligned}
$$

and the pseudo-inertia tensor $\widetilde{\mathbf{M}}$ only on $\boldsymbol{q}, \boldsymbol{u}, \boldsymbol{\Omega}_{\mathrm{E}}, \mathbf{F}$, and $\partial_{t} \mathbf{F}$, thus

$$
\begin{aligned}
& \tilde{\mathbf{M}}=\mathcal{M}_{\mathrm{b}} \mathbf{g}-\int_{\mathbb{R}^{1,3}}\left[\boldsymbol{x} \otimes \boldsymbol{x},\left[\mathbf{F}(\boldsymbol{q}+\boldsymbol{x}), \boldsymbol{\Omega}_{\mathrm{E}}\right]_{+}\right]_{+} f_{\mathrm{e}}(\|\boldsymbol{x}\|) \delta(\mathbf{u} \cdot \boldsymbol{x}) \mathrm{d}^{4} \boldsymbol{x} \\
& -\int_{\mathbb{R}^{1,3}} \mathbf{x} \otimes \mathbf{x}\left(\mathbf{x} \cdot \boldsymbol{\Omega}_{\mathrm{E}} \cdot \mathbf{F}(\boldsymbol{q}+\mathbf{x}) \cdot \mathbf{u}\right) f_{\mathrm{e}}(\|\mathbf{x}\|) \mathbf{u} \cdot \nabla_{\mathrm{g}} \delta(\mathbf{u} \cdot \mathbf{x}) \mathrm{d}^{4} \mathbf{x} \\
& -\int_{\mathbb{R}^{1,3}} \mathbf{F}(\boldsymbol{q}+\mathbf{x}) \cdot \mathbf{u} \otimes \mathbf{x} f_{\mathrm{e}}(\|\mathbf{x}\|) \delta(\mathbf{u} \cdot \mathbf{x}) \mathrm{d}^{4} \mathbf{x} .
\end{aligned}
$$

Remark: The first three terms in (8.27) are manifestly symmetric, but the fourth one is not and, as far as we can see, also cannot be symmetrized by adding a term whose action on $\stackrel{\circ}{\mathbf{u}}$ yields zero. Although this brings some technical inconveniences for concrete computations, it does not interfere with the remaining steps in our analysis.

At this point, the system of evolution equations (8.18), (8.20), (8.21), and (8.25) is already a quasi-explicit first-order system; however the tensor $\widetilde{\mathbf{M}}$ could be singular (as an operator acting by matrix multiplication (w.r.t. the Minkowski metric) on four-vectors). Our next step is to show that in typical situations $\widetilde{\mathbf{M}}$ is not singular. We will establish the invertibility of $\widetilde{\mathbf{M}}$ for admissible initial data in a large neighborhood of those data that correspond to the stationary solutions with empirical electron characteristics that covers essentially all physically sensible situations.

We begin with the observation that, since we have chosen a Lorentz frame for the initial value problem which coincides with the particle's initial rest frame, we can in particular choose initial data such that the particle and the fields remain stationary for all time. The stationary electric Coulomb field and the stationary gyro-magnetic field field, denoted here by an index ${ }_{s t}$, are defined by the equations

$$
-i \boldsymbol{\nabla} \times \mathcal{G}_{s t}(\boldsymbol{x})=4 \pi f_{\mathrm{e}}(|\boldsymbol{x}|) \boldsymbol{\omega}_{\mathrm{E}} \times \boldsymbol{x},
$$

with $\boldsymbol{\omega}_{\mathrm{E}}=\boldsymbol{\omega}_{\mathrm{E} 0}$ constant, and

$$
\boldsymbol{\nabla} \cdot \mathcal{G}_{s t}(\boldsymbol{x})=4 \pi f_{\mathrm{e}}(|\boldsymbol{x}|)
$$


together with the condition that $\mathcal{G}_{s t}(\boldsymbol{x}) \rightarrow \mathbf{0}$ as $|\boldsymbol{x}| \rightarrow \infty$. For a stationary situation in which the charge, gyro-magnetic moment, and renormalized mass are matched to those of the empirical electron, with $m_{\mathrm{b}}>0$ but small (details are given in section 10.1), the first and third field integrals in (8.27), evaluated with the corresponding $\mathbf{F}_{s t}$, are of magnitude $\alpha$ (Sommerfeld's fine structure constant) relative to the gyrational bare term, while the second field integral in (8.27), evaluated with the corresponding $\mathbf{F}_{s t}$, vanishes. The field integrals in (8.27) are therefore only small perturbations (in the mathematical sense of operator theory) to the gyrational bare term $\mathcal{M}_{\mathrm{b}} \mathbf{g}$ in $(8.27)$, which in turn is invertible because it acts just as multiplication by the strictly positive coefficient $\mathcal{M}_{\mathrm{b}}\left(\approx m_{\mathrm{e}}\right.$, the empirical electron mass) - recall that the metric tensor $\mathbf{g}$ acts as identity on four-vectors. Hence, we conclude that for a stationary, empirical data-honoring situation, $\widetilde{\mathbf{M}}$ is invertible.

Having established the invertibility of such a stationary $\widetilde{\mathbf{M}}$, we now turn to the discussion of initial data which do not launch a stationary solution. Since $\alpha<10^{-2}$, in such a nonstationary situation the tensor $\widetilde{\mathbf{M}}$ will remain invertible roughly up to the point where the field integrals in (8.27) become comparable to the gyrational bare term. Inspection of the field integrals reveals that this limit is reached (a) for applied initial field strengths about 100 times the surface field strength of the stationary electron, i.e. about $10^{8} \mathrm{~V} / \mathrm{m}$, or (b) for values of $-i \boldsymbol{\nabla} \times \mathcal{G}_{0}(\boldsymbol{x})-4 \pi f_{\mathrm{e}}(|\boldsymbol{x}|) \boldsymbol{\omega}_{\mathrm{E} 0} \times \boldsymbol{x}$ about $10^{2}$ times the surface field strength of the stationary electron per radial size of the electron. Interestingly, field configurations that strong are precisely those that saturate the admissibility condition (8.1). While therefore it is to be expected that a proof, if at all possible, of the general invertibility of $\widetilde{\mathbf{M}}$ for all $a$ priori admissible initial data will be a technically hard problem, from a physical perspective it is idle to worry about field strengths that strong within the Lorentz model. These critical field strengths are way outside the reasonable range of applied field strengths for which one can hope that the Lorentz model makes physically sensible predictions; whence, we will not address the issue of the critical field strengths any further and simply conclude with the observation that for essentially all physically acceptable initial data the tensor $\widetilde{\mathbf{M}}$ is a small perturbation of $\mathcal{M}_{\mathrm{b}} \mathbf{g}$ and therefore invertible.

We may now multiply (8.25) by the inverse of $\widetilde{\mathbf{M}}$ to obtain the first-order evolution equation

$$
\frac{\mathrm{d} \mathbf{u}}{\mathrm{d} \tau}=\widetilde{\mathbf{M}}^{-1} \cdot \widetilde{\boldsymbol{f}}
$$

valid surely initially and, if a continuously differentiable solution $\boldsymbol{u}$ of (8.25) exists, valid also for at least a short ensuing time interval during which $\widetilde{\mathbf{M}}$ will continue to be invertible, then, literally by continuity. Conversely, if (8.30) has a continuously differentiable solution $\boldsymbol{u}$, then as long as $\tilde{\mathbf{M}}$ remains invertible this solution will also solve (8.25). Equation (8.30) is called the hodograph equation for $\mathbf{u}$. It is of the desired format in $\stackrel{\circ}{\mathbf{u}}$.

\subsubsection{Consistency}

Before we address the question of how to solve our quasi-explicit system of evolution equations, a remark is in order regarding the following subtle point. Since $\mathbf{u} \cdot \stackrel{\circ}{\mathbf{u}}=0$, by taking the inner product of (8.30) we see that we must have $\mathbf{u} \cdot \widetilde{\mathbf{M}}^{-1} \cdot \widetilde{\boldsymbol{f}}=0$. Unfortunately, this 
last equation is not an automatic identity (as is, for instance, $\mathbf{u} \cdot \mathbf{F} \cdot \mathbf{u}=0$, which follows just by the anti-symmetry of $\mathbf{F}$, irrespective of whether $\boldsymbol{u}$ and $\mathbf{F}$ are dynamically related or not), and this may cause some concern. However, it is only necessary that $\boldsymbol{u} \cdot \widetilde{\mathbf{M}}^{-1} \cdot \widetilde{\boldsymbol{f}}$ vanishes identically for solutions to the dynamical equations, and this can be shown to be the case. In fact, it holds for any solution of (8.30) with given fields $\mathbf{F}$ and gyrograph $\boldsymbol{\Omega}_{\mathrm{E}}$ conditioned on $\tau \mapsto \Omega_{\mathrm{E}}(\tau)$ being a solution of (7.6). In brief, the apparent difficulty traces back to the fact that Nodvik's Minkowski force $\boldsymbol{f}$ given in (7.5) is generally not four-orthogonal to $\boldsymbol{u}$ [15] because it couples the spin-generated electromagnetic (dipole and higher multipole) moments to the inhomogeneities of the electromagnetic fields over the size of the particle. We have

$$
\boldsymbol{f} \cdot \mathbf{u}=-\int_{\mathbb{R}^{1,3}}(\boldsymbol{x}-\boldsymbol{q}) \cdot \boldsymbol{\Omega}_{\mathrm{E}} \cdot \mathbf{F}(\mathbf{x}) f_{\mathrm{e}}(\|\mathbf{x}-\boldsymbol{q}\|) \delta(\mathbf{u} \cdot(\mathbf{x}-\boldsymbol{q})) \mathrm{d}^{4} \mathbf{x} \cdot \mathbf{u},
$$

which vanishes identically (irrespective of F) only for a so-called 'particle without spin' for which $\boldsymbol{\Omega}_{\mathrm{E}} \equiv \mathbf{0}$ (more on particle models without spin in Appendices A.2 and A.3). This nonorthogonality of four-velocity and Minkowski force is countered on the left side of (7.3) by the spin-orbit coupling term $\mathbf{M}_{\mathrm{N}}$ in $\mathbf{M}$, which creates a generally anisotropic translational inertia of the gyrating charged particle so that the Minkowski momentum $\boldsymbol{p}$ and the four-velocity $\mathbf{u}$ are not anymore parallel to each other but related by the genuine tensor proportionality (7.4). By adapting the corresponding computations of Nodvik 15 to the present model, it can now be shown that the equation obtained by taking the inner product of (7.3) with $\mathbf{u}$ is identically satisfied conditioned on $\tau \mapsto \Omega_{\mathrm{E}}(\tau)$ being a solution of the gyrograph equations

(7.6), (7.7). The correspondingly conditioned identity $\mathbf{u} \cdot \widetilde{\mathbf{M}}^{-1} \cdot \widetilde{\boldsymbol{f}}=0$ now follows from the fact that 8.30 is equivalent to 7.3 ).

\subsubsection{Iterative treatment}

Having succeeded in writing the whole evolution problem as a regular quasi-explicit firstorder system (8.18), 8.20), (8.21), and (8.30), in the final step in our local analysis of the initial value problem we now explain that this regular quasi-explicit first-order system can be treated by a direct generalization of the familiar Picard iteration scheme. This then verifies that a unique regular solution exists and, above all, shows how it can actually be computed.

Thus, we consider the coupled sequences of approximate world-lines $\left\{\boldsymbol{q}^{(n)}(\tau)\right\}_{n=0}^{\infty}, \tau \geqslant 0$; approximate hodographs $\left\{\boldsymbol{u}^{(n)}(\tau)\right\}_{n=0}^{\infty}, \tau \geqslant 0$; approximate gyrographs $\left\{\boldsymbol{\Omega}_{\mathrm{E}}{ }^{(n)}(\tau)\right\}_{n=0}^{\infty}, \tau \geqslant 0$; and approximate electromagnetic fields $\left\{\mathcal{G}^{(n)}(\boldsymbol{x}, t)\right\}_{n=0}^{\infty}$, with $\boldsymbol{x} \in \mathbb{R}^{3}$ and $t \geqslant 0$, jointly defined by

$$
\begin{aligned}
& \frac{\mathrm{d}}{\mathrm{d} \tau} \boldsymbol{q}^{(n+1)}=\mathbf{u}^{(n)}, \\
& \frac{\mathrm{d}}{\mathrm{d} \tau} \mathbf{u}^{(n+1)}=\left(\widetilde{\mathbf{M}}^{-1}\right)^{(n)} \cdot \widetilde{\boldsymbol{f}}^{(n)}, \\
& \frac{\mathrm{d}}{\mathrm{d} \tau} \mathbf{S}_{\mathrm{b}}{ }^{(n+1)}=-\left[\boldsymbol{\Omega}_{\mathrm{FW}}{ }^{(n)}, \mathbf{S}_{\mathrm{b}}{ }^{(n)}\right]_{-}+\mathbf{t}^{(n)},
\end{aligned}
$$


and

$$
\partial_{t} \mathcal{G}^{(n+1)}(\boldsymbol{x}, t)=-i \boldsymbol{\nabla}_{\gamma} \times(\psi \mathcal{G})^{(n)}(\boldsymbol{x}, t)-4 \pi(\psi \boldsymbol{j})^{(n)}(\boldsymbol{x}, t),
$$

together with the initial-time constraint

$$
\boldsymbol{\nabla} \cdot \mathcal{G}^{(n+1)}(\boldsymbol{x}, 0)=4 \pi \rho^{(n)}(\boldsymbol{x}, 0)
$$

starting the iteration with functions that are constant in time,

$$
\begin{aligned}
\mathcal{G}^{(0)}(\boldsymbol{x}) & =\mathcal{G}_{0}(\boldsymbol{x}) \\
\boldsymbol{q}^{(0)} & =\boldsymbol{O} \\
\boldsymbol{u}^{(0)} & =\boldsymbol{e}_{0} \\
\mathbf{S}_{\mathrm{b}}{ }^{(0)} & =\mathcal{I}_{\mathrm{b}}\left(\left|\boldsymbol{\omega}_{\mathrm{E} 0}\right|\right) \omega_{\mathrm{E} 0} \boldsymbol{e}_{1} \wedge \boldsymbol{e}_{2},
\end{aligned}
$$

and with correspondingly vanishing time derivatives of the starting data,

$$
\begin{aligned}
\partial_{t} \mathcal{G}^{(0)}(\mathbf{x}) & =\mathbf{0} \\
\stackrel{\circ}{q}^{(0)} & =\boldsymbol{O} \\
\stackrel{\circ}{\mathbf{u}}^{(0)} & =\boldsymbol{O} \\
\stackrel{\circ}{\mathbf{S}}_{\mathrm{b}}^{(0)} & =\mathbf{0} .
\end{aligned}
$$

This set of equations determines the first-order time derivatives of the maps $t \mapsto \mathbf{F}^{(n+1)}$, $\tau \mapsto \boldsymbol{q}^{(n+1)}, \tau \mapsto \boldsymbol{u}^{(n+1)}$, and $\tau \mapsto \mathbf{S}_{\mathrm{b}}{ }^{(n+1)}$, in terms of the order $n$ maps $t \mapsto \mathcal{G}^{(n)}, \tau \mapsto \boldsymbol{q}^{(n)}$, $\tau \mapsto \mathbf{u}^{(n)}$, and $\tau \mapsto \mathbf{S}_{\mathrm{b}}{ }^{(n)}$, and the first-order time derivatives of the order $n$ maps. The maps $t \mapsto \mathbf{F}^{(n+1)}, \tau \mapsto \boldsymbol{q}^{(n+1)}, \tau \mapsto \mathbf{u}^{(n+1)}$, and $\tau \mapsto \mathbf{S}_{\mathrm{b}}{ }^{(n+1)}$ themselves are now obtained by simply integrating the whole system with respect to the respective time variables. This is done for the Maxwell-Lorentz equations (8.35), (8.36) by the standard Laplace transform technique, thus picking up the consistent initial data $\mathcal{G}_{0}$ (i.e. $\mathbf{F}_{0}$ ) posed at $t=0$ through the familiar integration by parts on $\partial_{t} \mathcal{G}$. Equations (8.32), (8.33), and (8.34) are simply integrated w.r.t. the proper-time from $\tau=0$ to $\tau$, thereby picking up the initial data $\boldsymbol{q}(0)$, $\mathbf{u}(0)$, and $\mathbf{S}_{\mathrm{b}}(0)$ (viz. $\boldsymbol{\Omega}_{\mathrm{E}}(0)$ ) from the integration of the explicit proper-time derivatives in the respective left-hand sides. This completes the $n+1^{\text {st }}$ iteration cycle. Starting with the prescribed order zero maps (8.40) and their derivatives (8.44), one can thus systematically proceed to higher order iterates. The upshot is an algorithm for the practical computation of the solution, provided the iteration converges.

Remark: It is important to notice that "practical computation" means to be able to obtain an approximate solution to any degree of precision in some finite region of spacetime. To reach this goal it is not necessary to actually compute the global iterands in spacetime (which would be impossible practically). A finite number of iterations on a nested, decreasing sequence of spacetime regions, starting with a sufficiently big initial region, will suffice. 


\subsubsection{Some remarks on rigorous results}

The convergence of our algorithm is equivalent to a contraction principle for the fixed-point map in the global future particle histories starting with the specified Cauchy data, their time derivatives (treated as independent functions in the iteration and in the fixed point map), and the field decorations of spacetime in the future of $\Sigma_{0}$, starting with the initial field configuration on $\Sigma_{0}$. Proving the contraction principle establishes the convergence of the iteration to a unique global solution in the future (and under similar conditions also the past) of the initial time for those admissible Cauchy data for which the tensor $\tilde{\mathbf{M}}$ remains a small perturbation of $\mathcal{M}_{\mathrm{b}} \mathbf{g}$ and therefore invertible.

So far, for the model in which the bare rest mass and the charge are both concentrated on the same sphere, and provided the ratio of electrostatic to bare rest mass is smaller than 1 , we proved global existence and uniqueness for the iteration algorithm in the special case that only the particle's spin degrees of freedom participate in the particle + field dynamics. In the further specialization that the axis of rotation remains fixed, we were able to prove exponentially fast approach to a stationary gyration state for the field-particle system, convergence understood on families of nested compact sets, superposed on which is an outgoing field of electromagnetic radiation. For the proofs of these results, see 33].

For more general evolutions, convergence has not yet been established. The dynamical equations of our massive LED may spoil the global existence of the dynamics in several ways. First, the equatorial rotation speed has to remain subluminal for a particle with finite bare rest mass $m_{\mathrm{b}}$. (As we will see later on, the situation is different in the limit $m_{\mathrm{b}} \rightarrow 0$ where the rotation speed can reach the speed of light, or actually is locked in at that speed). Second, the acceleration of the particle has to remain below a critical threshold (mentioned above) to guarantee an ordered particle history. Third, but only relevant to the multi-particle systems not considered here, any two particles have to have a minimum distance between them to avoid overlapping cutoffs. We now comment on the first two problems.

The first problem can be controlled by an appropriate a priori choice of the bare rest mass distribution. It suffices to discuss two representative choices, volume and surface inertia. They differ in their limiting behavior when $\left\|\boldsymbol{w}_{\mathrm{E}}\right\| \rightarrow 1 / R$. The volume choice for $f_{\mathrm{m}}$ is representative for the larger class of continuous functions $f_{\mathrm{m}} \in C^{0}([0, R])$, in all of which cases $\lim _{\left\|\boldsymbol{w}_{\mathrm{E}}\right\| \rightarrow 1 / R} \mathcal{M}_{\mathrm{b}}\left(\left\|\boldsymbol{w}_{\mathrm{E}}\right\|\right)<\infty$. In such a case it takes only a finite amount of gyrational energy to bring an equatorial point to the speed of light. This signals that the choice of a continuous $f_{\mathrm{m}}$ will create a mathematically delicate problem when it comes to the question whether the gyrational dynamics remains subluminal, and so one may expect that this choice may easily lead to a singularity in finite time. In contrast, for the surface inertia choice for $f_{\mathrm{m}}$, the function $\mathcal{M}_{\mathrm{b}}$ is not only increasing and convex, but satisfies $\mathcal{M}_{\mathrm{b}}\left(\left\|\mathbf{w}_{\mathrm{E}}\right\|\right) \rightarrow \infty$ as

$\left\|\mathbf{w}_{\mathrm{E}}\right\| \rightarrow 1 / R$. In this case it would require an infinite amount of gyrational energy to bring an equatorial point to the speed of light, so that it is to be expected that the dynamics will not lead to a singularity in finite time. These considerations show that surface inertia is preferable, although we do not claim that this requirement is necessary for global or even local existence.

The second problem is much harder to control a priori. For a moving particle one now has to rule out that constructive interference of electromagnetic radiation fields leads to a 
violation of the acceleration limit (in our Lorentz frame)

$$
|\ddot{\boldsymbol{q}}|<\frac{1}{R \gamma^{3}}
$$

where $R$ is the radius of the particle and $\gamma=1 / \sqrt{1-\dot{\boldsymbol{q}}^{2}}$. At the initial instant $t=\tau=0$, the particle is at rest and the estimate (8.45) reduces to (8.1).

Notice finally that the smallness condition that we need in our proof in [33] is surely going to be violated if one lets the bare rest mass flow to zero. The control of this situation apparently requires completely different techniques.

Due to all these technical difficulties, the global existence and uniqueness of regular solutions, at least for the physically relevant parameter range of the model, still awaits its verification. In the following sections we proceed under the assumption that the dynamics of the field-particle system is globally well-defined. Put differently, whatever is concluded in those sections applies only to those situations for which a unique solution exists for all time.

\section{$9 \quad$ Scattering}

Having established that the dynamical state in our single-particle massive LED in spacetime $\mathbb{R}^{1,3}$ is given by the transversal part of $\mathcal{G}$ (viz., $\mathbf{F}$ ) on a spacelike hypersurface of spacetime containing the particle support, together with the particle variables $\boldsymbol{q}, \boldsymbol{u}$, and $\boldsymbol{\Omega}_{\mathrm{E}}$ (or $\mathbf{S}_{\mathrm{b}}$ ) coincidental with the particle support in the spacelike hypersurface, we now turn the important question which of these states are scattering states, and in which sense. Loosely speaking, a scattering state is a state whose backward evolution tends asymptotically in the infinite past to a superposition of free evolutions of propagating (in space) field and particle degrees of freedom, and similarly its forward evolution tends asymptotically in the infinite future to a(nother) superposition of free evolutions of propagating field and particle degrees of freedom. The scattering problem is concerned with establishing the existence of the scattering states, with their unique identification, and further with their classification according as to how their asymptotically free evolution in the infinite past is connected with their asymptotically free evolution in the infinite future.

We will limit our discussion to situations in which the conserved quantities $\boldsymbol{P}, \mathbf{L}$, and $s^{2}$ are finite. Moreover, we take a foliation in which $\boldsymbol{P}=(M, \mathbf{0})$, i.e. a center of mass frame for the field-particle system. We assume the center of mass is at the origin of the initial leaf.

We expect that in our massive LED there are basically three categories of states, namely the non-propagating (a.k.a. bound) states, the scattering states, and the 'catastrophic states'. For the catastrophic states the evolution either terminates at a finite time, or blows up in infinity time, or suffers some other pathological behavior like loss of uniqueness. As explained in the previous section, the global existence and uniqueness problem is largely unsettled so that we will not be able to rule out catastrophic states nor characterize them as physically irrelevant (although we expect the latter to be true). However, the special global existence and uniqueness result in [33] shows that for a certain class of initial conditions the evolution does exist globally, and these states are in fact scattering states. We shall show in this section that the scattering states have the remarkable, and in fact physically 
indispensable character of soliton dynamics for the renormalized particle degrees of freedom. As for the bound states, we readily characterize the stationary states, but we have no control yet on the question of whether periodic (or perhaps quasi-periodic) non-propagating states exist as well.

This section on scattering is mainly included for the purpose of demonstrating the important solitonic character of the renormalized particle dynamics in scattering situations. As such, we will necessarily be brief on the other scattering issues.

As a general technical reference to scattering theory we recommend [37], though most of the emphasis there is on quantum mechanical potential scattering; in this context, see also [38, 39] for subsequent technical breakthroughs, and 40] for a very clear exposition of the conceptual issues involved and further references. Various classical scattering results in electrodynamics are discussed in [26].

\subsection{The bound states}

Massive LED describes the interacting dynamics of two a priori subsystems, the charged bare particle and the electromagnetic field, each of which is characterized by its own dynamical degrees of freedom. With only a single charged bare particle interacting with the electromagnetic field in otherwise empty space, it could seem that there are no bound states. However, some of the particle's spin degrees of freedom couple strongly to some of the dynamical degrees of freedom of the electromagnetic field, as a result of which bound states exist. The most elementary ones give rise to the notion of the renormalized particle. In addition, more subtle scenarios may possibly lead to dynamical bound states, on which we comment only briefly.

\subsubsection{The stationary bound states}

The most obvious bound states are stationary solutions with nonvanishing particle spin. Clearly we can choose the standard foliation of spacetime for these states, i.e. a Lorentz frame $\mathcal{F}_{\mathrm{L}}$ in which the particle is at rest at the origin of the space slice $\Sigma_{0} \sim \Sigma_{t}$; i.e. $\boldsymbol{q}(\tau)=\boldsymbol{O}$ for all $\tau$ so that $\boldsymbol{u}(\tau)=\boldsymbol{e}_{0}$. The stationary states compatible with our conventions made in the Cauchy problem have been defined earlier; all other stationary bound states that satisfy our convention about the center of mass are now obtained by at most a rotation from these stationary states.

For later convenience, we here list the solutions explicitly. The stationary particle in the center of mass frame is spinning with constant angular velocity tensor $\Omega_{\mathrm{E}}$. The stationary charge-current density four-vector thus reads

$$
\boldsymbol{J}_{s t}(\mathbf{x})=\left(\boldsymbol{e}_{0}-\boldsymbol{\Omega}_{\mathrm{E}} \cdot \mathbf{x}\right) f_{\mathrm{e}}(\|\mathbf{x}\|) .
$$

We seek a stationary solution of the vector wave equation $\square \boldsymbol{A}_{s t}=\boldsymbol{J}_{s t}$ satisfying the Lorentz gauge condition and vanishing at spatial infinity. Recalling that $-\boldsymbol{\Omega}_{\mathrm{E}} \cdot \boldsymbol{X}=\left(0, \boldsymbol{\omega}_{\mathrm{E}} \times \boldsymbol{x}\right)$, and introducing the time-space decompositions for the current density $\boldsymbol{J}=(\rho, \boldsymbol{j})$ and electromagnetic potential four-vector $\boldsymbol{A}=(\phi, \mathcal{A})$, for the stationary Coulomb potential $\phi_{s t}(\boldsymbol{x})$ we 
find

$$
\phi_{s t}(\boldsymbol{x})=\int_{\mathbb{R}^{3}} \frac{1}{\left|\boldsymbol{x}-\boldsymbol{x}^{\prime}\right|} f_{\mathrm{e}}\left(\left|\boldsymbol{x}^{\prime}\right|\right) \mathrm{d}^{3} x^{\prime}
$$

and for the stationary vector potential $\mathcal{A}_{s t}(\boldsymbol{x})$,

$$
\mathcal{A}_{s t}(\boldsymbol{x})=\boldsymbol{\omega}_{\mathrm{E}} \times \int_{\mathbb{R}^{3}} \frac{\boldsymbol{x}^{\prime}}{\left|\boldsymbol{x}-\boldsymbol{x}^{\prime}\right|} f_{\mathrm{e}}\left(\left|\boldsymbol{x}^{\prime}\right|\right) \mathrm{d}^{3} \boldsymbol{x}^{\prime} .
$$

The electric and magnetic fields are obtained in the usual manner as $\mathcal{E}_{s t}(\boldsymbol{x})=-\boldsymbol{\nabla} \phi_{s t}(\boldsymbol{x})$ and $\mathcal{B}_{s t}(\boldsymbol{x})=\boldsymbol{\nabla} \times \mathcal{A}_{s t}(\boldsymbol{x})$, so that

$$
\mathcal{G}_{s t}(\boldsymbol{x}, t)=-\boldsymbol{\nabla} \phi_{s t}(\boldsymbol{x})+i \boldsymbol{\nabla} \times \mathcal{A}_{s t}(\boldsymbol{x}) .
$$

Since we chose a spherical distribution $f_{\mathrm{e}}(\mid$. $\mid)$ with compact support in $\mathbb{R}^{3}$, the Coulomb and the vector potential take a universal form outside the support of the particle, given by

$$
\left.\begin{array}{c}
\phi_{s t}(\boldsymbol{x})=-e \frac{1}{|\boldsymbol{x}|} \\
\mathcal{A}_{s t}(\boldsymbol{x})=\boldsymbol{\mu} \times \frac{\boldsymbol{x}}{|\boldsymbol{x}|^{3}}
\end{array}\right\} \quad \text { for } r>R,
$$

where $\boldsymbol{\mu}$ is the magnetic moment for the stationary $\boldsymbol{\omega}_{\mathrm{E}}$,

$$
\boldsymbol{\mu}=\frac{1}{2} \int_{\mathbb{R}^{3}} \boldsymbol{x} \times\left(\boldsymbol{\omega}_{\mathrm{E}} \times \boldsymbol{x}\right) f_{\mathrm{e}}(|\boldsymbol{x}|) \mathrm{d}^{3} x .
$$

Clearly, away from its support the stationary charged particle's electromagnetic signature is that of an electric point charge and a magnetic point dipole, as was to be anticipated.

Finally, we remark that the static limit $\left|\boldsymbol{\omega}_{\mathrm{E}}\right| \rightarrow 0$ for the stationary bound state is no longer a bound state of the field-particle system, in the sense that only the passive Coulomb field remains attached to the charged bare particle.

\subsubsection{The non-stationary bound states}

In various approximate, linearized versions of (massive) LED, time-periodic non-propagating field-particle solutions have been found. Time-periodic solutions do not exist if the Wiener condition is satisfied, viz. the Fourier transform of $f_{\mathrm{e}}$ be strictly positive. However, notice that neither the surface density nor the volume density discussed in this paper satisfy the Wiener condition. In principle one should therefore be prepared for the possibility of periodic solutions in our massive LED. On the other hand, rigorous studies of simpler semi-relativistic models of a particle interacting with a scalar wave field show that time-periodic bound states of their linearized version are structurally unstable to the nonlinear perturbation of the linear dynamics [41]; see also [42]. No such study has yet been carried out for our massive LED. 


\subsection{Scattering states}

In our center of mass initial leaf, we can divide the scattering states into two categories: (a) scattering of electromagnetic radiation off a non-moving particle, and (b) scattering of radiation and moving particle in an encounter.

\subsubsection{Scattering with a non-moving particle}

Scattering with a non-moving particle is considerably simpler because the family of space slices of simultaneity for the particle are just the standard foliation of spacetime, as for a stationary particle. The only dynamically active degree of freedom of the particle is its Euler tensor, or in the stationary Lorentz frame of our foliation, the angular velocity vector $\boldsymbol{\omega}_{\mathrm{E}}(t)$. The dynamical degrees of freedom of the electromagnetic field are restricted to be compatible with the condition that the particle sit still at the origin of space. For instance, large classes of axisymmetric field decorations of spacetime will be fine. This case has been studied in by us in rigor; details will be given in [33]. Here we summarize the main results, obtained under the condition that the ratio of the particle's electrostatic Coulomb energy to the bare rest mass is smaller than 1 .

The best understood situation is when the particle's axis of rotation does not change during the evolution, say $\boldsymbol{\omega}_{\mathrm{E}}=\omega_{\mathrm{E}} \boldsymbol{e}_{3}$, so that $\omega_{\mathrm{E}}$ is the only remaining dynamical particle degree of freedom. For all admissible initial conditions corresponding to these restrictions, we know that on families of nested compact sets the field-particle system converges exponentially fast to a stationary state which is a bound state if the conserved $s^{2} \neq 0$ initially and the static state if $s^{2}=0$, superposed on which in either case is an outgoing field of electromagnetic radiation. The evolution of the electromagnetic field is thus given by the scattering formula

$$
\mathcal{G}(\boldsymbol{x}, t) \stackrel{t \rightarrow+\infty}{\longrightarrow} \mathcal{G}_{s t}(\boldsymbol{x})+e^{-i t \nabla \times} \mathcal{G}_{\mathrm{rad}}^{\text {out }}(\boldsymbol{x})
$$

where $\mathcal{G}_{\text {rad }}^{\text {out }}$ is a divergence-free electromagnetic field, orthogonal to all bound-state fields, uniquely determined by the initial state, characterizing the outgoing radiation. Similarly

$$
\mathcal{G}^{*}(\boldsymbol{x}, t) \stackrel{t \rightarrow-\infty}{\longrightarrow} \mathcal{G}_{s t}^{*}(\boldsymbol{x})+e^{i t \nabla \times} \mathcal{G}_{\mathrm{rad}}^{\text {in }}{ }^{*}(\boldsymbol{x})
$$

where ${ }^{*}$ denotes complex conjugate. We remark that $\mathcal{G}_{s t}$ is the same stationary bound state in both formulas, which is a simple illustration of the soliton dynamics of the renormalized particle in this special situation.

In the general non-moving particle case where the axis of $\boldsymbol{\omega}_{\mathrm{E}}$ is not fixed during the evolution, we know that every admissible initial state compatible with the constraints evolves uniquely into the future (respectively, past), but we do not yet know that on families of nested compact sets the field-particle system converges (exponentially fast) to a stationary state. However, we do conjecture that this will be the case. Assuming this to be true we were able to show that for large times the evolution of the electromagnetic field satisfies the scattering formulas

$$
\mathcal{G}(\boldsymbol{x}, t) \stackrel{t \rightarrow+\infty}{\longrightarrow} \mathcal{G}_{\text {st }}^{\text {out }}(\boldsymbol{x})+e^{-i t \nabla \times} \mathcal{G}_{\mathrm{rad}}^{\text {out }}(\boldsymbol{x})
$$


and

$$
\mathcal{G}^{*}(\boldsymbol{x}, t) \stackrel{t \rightarrow-\infty}{\longrightarrow} \mathcal{G}_{s t}^{\text {in } *}(\boldsymbol{x})+e^{i t \nabla \times} \mathcal{G}_{\mathrm{rad}}^{\text {in }}{ }^{*}(\boldsymbol{x})
$$

where now $\mathcal{G}_{s t}^{\text {in }}$ and $\mathcal{G}_{s t}^{\text {out }}$ are generally not the same stationary bound state; however, they differ by at most a space rotation, and this once again illustrates the soliton dynamics of the renormalized particle.

In both situations, the explicit characterization of the scattering operator from the "in" states to the "out" states has not yet been worked out.

\subsubsection{Scattering with a moving particle}

Scattering with a moving particle can mean any scattering process in which the particle moves for at least a finite interval of time. In that case we need to work with a nonstandard foliation, as explained in the section on the Cauchy problem for the state. Needless to say, so far this is the least explored case. The explicit characterization of the scattering states, and of the scattering operator from the "in" states to the "out" states still have to be worked out.

However, although it is rigorously unknown which initial states are scattering states for which the particle moves, a priori speaking there are several types of scattering processes to be distinguished: (A) the particle moves only a finite distance, associated with which are bound states in the distant past and future - this is a perturbation of the previous "scattering of radiation off a bound state;" (B) the particle is at rest only in the past (respectively, only in the future) but moves freely in the asymptotic future (respectively, past); (C) the particle moves freely in the asymptotic past and future. Furthermore, one can give the following partial characterization of the "in" and "out" states.

In the situations (A) and the parts of (B) associated with a stationary bound state, the scattering asymptotics differs from the one discussed in the previous subsection by at most a translation of the stationary bound state.

In the situations $(\mathrm{C})$ and the parts of $(\mathrm{B})$ not associated with a stationary bound state, the scattering asymptotics in the specified foliation is given as follows. As for the asymptotic particle motion, all the "in" states $\left\{\boldsymbol{q}^{\text {in }}, \boldsymbol{u}^{\text {in }}, \boldsymbol{\Omega}_{\mathrm{E}}{ }^{\text {in }}\right\}$, as well as all the "out" states $\left\{\boldsymbol{q}^{\text {out }}, \boldsymbol{u}^{\text {out }}, \Omega_{\mathrm{E}}{ }^{\text {out }}\right\}$, are obtained from the stationary particle states considered above by the action of an element of the Poincaré group, more explicitly a combination of a translation, a rotation, and a Lorentz boost. The free evolution of a particle is obtained by applying the Lorentz-time propagation operator to these states, or expressed in terms of proper-time $\tau$ (recall that $q^{0}(\tau)=t$ ),

$$
\begin{aligned}
& \boldsymbol{q}(\tau) \stackrel{\tau \rightarrow+\infty}{\longrightarrow} \boldsymbol{q}^{\text {out }}+\boldsymbol{u}^{\text {out }} \tau \\
& \mathbf{u}(\tau) \stackrel{\tau \rightarrow+\infty}{\longrightarrow} \boldsymbol{u}^{\text {out }} \\
& \boldsymbol{\Omega}_{\mathrm{E}}(\tau) \stackrel{\tau \rightarrow+\infty}{\longrightarrow} \boldsymbol{\Omega}_{\mathrm{E}}^{\text {out }}
\end{aligned}
$$


and correspondingly for $\tau \rightarrow-\infty$ using the "in" states. Associated with these free asymptotic evolution of the particle are the co-moving electromagnetic fields. Sufficiently far away from the particle these are just co-moving in the standard foliation to which the compatible foliation is asymptotically close except in a neighborhood of the world-tube. Their electromagnetic "in" and "out" states are parameterized by the space part $\boldsymbol{x}^{\text {out }}$ of $\boldsymbol{q}^{\text {out }}$, the constant velocity $\boldsymbol{v}^{\text {out }}$ in $\boldsymbol{u}^{\text {out }}=(\gamma, \gamma \boldsymbol{v})^{\text {out }}$, and the angular velocity vector $\boldsymbol{\omega}_{\mathrm{E}}{ }^{\text {out }}$ corresponding to $\Omega_{\mathrm{E}}{ }^{\text {out }}$. To have a handy abbreviation for this parameter set, we choose the index com for 'co-moving.' We set

$$
\begin{gathered}
\phi_{\mathrm{com}}^{\text {out }}(\boldsymbol{x}) \stackrel{\text { def }}{=} \frac{1}{\left|y_{\|} \hat{\boldsymbol{v}}+\sqrt{1-\boldsymbol{v}^{2}} \boldsymbol{y}_{\perp}\right|}+\left(1-\boldsymbol{v}^{2}\right) \frac{\boldsymbol{v} \cdot(\boldsymbol{\mu} \times \boldsymbol{y})}{\left|y_{\|} \hat{\boldsymbol{v}}+\sqrt{1-\boldsymbol{v}^{2}} \boldsymbol{y}_{\perp}\right|^{3}} \\
\mathcal{A}_{\mathrm{com}}^{\text {out }}(\boldsymbol{x}) \stackrel{\text { def }}{=} \frac{\boldsymbol{v}}{\left|y_{\|} \hat{\boldsymbol{v}}+\sqrt{1-\boldsymbol{v}^{2}} \boldsymbol{y}_{\perp}\right|}+\left(1-\boldsymbol{v}^{2}\right) \frac{\boldsymbol{\mu} \times\left(\gamma y_{\|} \hat{\boldsymbol{v}}+\boldsymbol{y}_{\perp}\right)}{\left|y_{\|} \hat{\boldsymbol{v}}+\sqrt{1-\boldsymbol{v}^{2}} \boldsymbol{y}_{\perp}\right|^{3}}
\end{gathered}
$$

with

$$
\hat{\boldsymbol{v}} \stackrel{\text { def }}{=} \boldsymbol{v} /|\boldsymbol{v}|, \quad y_{\|} \stackrel{\text { def }}{=}\left(\boldsymbol{x}-\boldsymbol{x}^{\text {out }}\right) \cdot \hat{\boldsymbol{v}}, \quad \boldsymbol{y}_{\perp}=\boldsymbol{x}-\boldsymbol{x}^{\text {out }}-y_{\|} \hat{\boldsymbol{v}}
$$

and where $\boldsymbol{v}=\boldsymbol{v}^{\text {out }}$ and $\boldsymbol{\mu}=\boldsymbol{\mu}^{\text {out }}$. After these preparations, we can give the asymptotic future evolution of the electromagnetic field for such a scattering state as

$$
\begin{aligned}
\mathcal{G}(\boldsymbol{x}, t) \stackrel{t \rightarrow+\infty}{\longrightarrow} & -\nabla\left(e^{-t \boldsymbol{v}^{\text {out }} \cdot \nabla} \phi_{\mathrm{com}}^{\text {out }}(\boldsymbol{x})\right)-\left(\partial_{t}-i \nabla \times\right)\left(e^{-t \boldsymbol{v}^{\text {out }} \cdot \nabla} \mathcal{A}_{\mathrm{com}}^{\text {out }}(\boldsymbol{x})\right) \\
& +e^{-i t \nabla \times} \mathcal{G}_{\mathrm{rad}}^{\text {out }}(\boldsymbol{x})
\end{aligned}
$$

Similar formulas hold for the asymptotic past evolution in terms of the "in" potentials.

\subsection{Soliton dynamics}

While the rigorous treatment of the scattering problem of massive LED is still in its infancy, in all instances of scattering a remarkable conclusion about the gyrational particle degrees of freedom can be made. Naïvely one would expect that generally the orientation and the norm of the angular velocity of the particle would change during the scattering processand with it the rotational and renormalized mass of the particle, its spin and magnetic moment. However, precisely this does not happen. Any scattering process connects two freely evolving particle states with identical renormalized mass and identical magnitudes of spin and magnetic moment. The relativistic Lorentz electron, equipped with non-vanishing positive bare inertia, thus has the dynamical characteristics of an elementary particle in the best sense one could have hoped for in a classical theory.

By adapting the terminology of Spohn [43] and collaborators, who considered charged particles without spin, we call the renormalized particle a spinning charge soliton. As remarkable as this soliton dynamics itself, is the fact that it is a simple joint consequence of the conservation of $s^{2}=-(1 / 2) \operatorname{Tr} \mathbf{S}^{\perp} \cdot \mathbf{S}^{\perp}$ together with the fact that $\mathbf{S}^{\perp}=\mathbf{S}$ for a stationary bound state, and the invertibility of the map $\Omega_{\mathrm{E}} \mapsto \mathbf{S}$ for such a state. Indeed, we need only consider a stationary bound state, for we know that the asymptotic future (past) evolution 
of the scattered particle dressed with its co-moving fields (the renormalized particle) is just a translated and boosted such state.

Explicitly, for a stationary bound state we have, in the Lorentz frame and in dual vector notation,

$$
\boldsymbol{s}=\int_{\mathbb{R}^{3}} \frac{\boldsymbol{x} \times\left(\boldsymbol{\omega}_{\mathrm{E}} \times \boldsymbol{x}\right)}{\sqrt{1-\left|\boldsymbol{\omega}_{\mathrm{E}} \times \boldsymbol{x}\right|^{2}}} f_{\mathrm{m}}(|\boldsymbol{x}|) \mathrm{d}^{3} x+\int_{\mathbb{R}^{3}} \boldsymbol{x} \times \mathcal{A}_{s t}(\boldsymbol{x}) f_{e}(|\boldsymbol{x}|) \mathrm{d}^{3} x,
$$

the first term being the bare spin, the second the electromagnetic spin of the particle. For the bare spin $\boldsymbol{s}_{\mathrm{b}}$ we already saw, in our section on the Cauchy problem, that it is parallel to $\boldsymbol{\omega}_{\mathrm{E}}$ and the map $\boldsymbol{s}_{\mathrm{b}} \mapsto \boldsymbol{\omega}_{\mathrm{E}}$ invertible. Using our formula (9.3) for the stationary vector potential of a bound state, we see by explicit computation that also the total spin $\boldsymbol{s}$ is parallel to $\boldsymbol{\omega}_{\mathrm{E}}$, and the map $\boldsymbol{s} \mapsto \boldsymbol{\omega}_{\mathrm{E}}$ is invertible. We have $\boldsymbol{s}=I\left(\left|\boldsymbol{\omega}_{\mathrm{E}}\right|\right) \boldsymbol{\omega}_{\mathrm{E}}$, with $I>0$ and map $x \mapsto x I(x)$ increasing, vanishing for $x=0$. Now, $|\boldsymbol{s}|=\left|\boldsymbol{\omega}_{\mathrm{E}}\right| I\left(\left|\boldsymbol{\omega}_{\mathrm{E}}\right|\right)$, so that from the invertibility of $x \mapsto x I(x)$ we conclude that $|\boldsymbol{s}|$ determines $\left|\boldsymbol{\omega}_{\mathrm{E}}\right|$ uniquely in a stationary bound state. Thus, from the constancy of $s^{2}$ we conclude that $\left|\boldsymbol{\omega}_{\mathrm{E}}\right|$ in the asymptotic future state has the same value as in the asymptotic past state. The solitonic character of the renormalized particle is proved.

\section{The limit of vanishing bare rest mass}

We carry out the renormalization group analysis for a simple example where the various dynamical functions can be computed explicitly. Specifically, we here consider 'densities' $f_{\mathrm{m}}$ and $f_{\mathrm{e}}$ which are Dirac measures concentrated on the surface of a two-sphere of radius $R$, viz.

$$
\frac{1}{m_{\mathrm{b}}} f_{\mathrm{m}}(|\boldsymbol{x}|)=\frac{1}{-e} f_{\mathrm{e}}(|\boldsymbol{x}|)=\frac{1}{4 \pi R^{2}} \delta(|\boldsymbol{x}|-R)
$$

The elementary charge $e$ will not be tempered with and therefore not displayed as variable in the arguments of the functions below. Beside having set the charge $-e$ of the model particle equal to that of the real electron, we also demand that its total model mass $m$ matches the empirical electron mass, $m=m_{\mathrm{e}}$, and that its magnetic moment $|\mu|$ matches the empirical electron magnetic moment, $|\mu|=\mu_{\mathrm{e}}$.

Remark: In this and the next section we resort to units in which the speed of light $c$ is displayed explicitly, to facilitate the comparison with the formulas used in physical data books.

The renormalized particle spin will be derived in our model in the limit of vanishing bare rest mass, whence is not to be matched to a 'corresponding empirical value,' but rather compared. Naïvely speaking it is to be expected that in our classical theory with ultraviolet cutoff the spin-to-magnetic-moment relation

$$
|\mu|=g \frac{e}{2 m_{\mathrm{e}} c} s
$$

with Landé factor $g \approx g_{\mathrm{Cl}}=1$ should hold. Interestingly, we will find $g \approx 2 / 3$. 
We remark that, without further elaboration, there remains a certain ambiguity in matching our stationary model data to empirical electron data inferred with the help of quantum mechanics from data of dynamical experiments, viz. $m_{\mathrm{e}}$ in scattering experiments, and $\mu_{\mathrm{e}}$ in resonance frequency experiments in a Penning trap. The ultimate justification for these choices has to wait for some future dynamical analysis of our model.

\subsection{The limit $m_{\mathbf{b}} \rightarrow 0^{+}$for the stationary electron}

By performing at most a space translation, we can assume that the stationary particle, and its self-fields, are centered the at the origin of the space slice $\mathbb{R}^{3}$ of the Lorentz frame in which the system is stationary. Since the time-component of the spin four-vector and the angular velocity four-vector vanish in our stationary frame, after at most a space rotation we can furthermore assume that the spin four-vector of the stationary bare particle, $\mathbf{s}_{\mathrm{b}}$, and of its self-fields, $\boldsymbol{s}_{\mathrm{f}}$, points along the $\boldsymbol{e}_{3}$ direction. Since $\boldsymbol{e}_{3}=\left(0, \boldsymbol{e}_{3}\right)$, we can canonically identify these spin four-vectors with the familiar bare and field spin three-vectors $\boldsymbol{s}_{\mathrm{b}}, \boldsymbol{s}_{\mathrm{f}}$, respectively the angular velocity four-vector with the angular velocity three-vector $\boldsymbol{\omega}_{\mathrm{E}}$, which moreover can be written as $\boldsymbol{s}_{\mathrm{b}}=s_{\mathrm{b}} \boldsymbol{e}_{3}, \boldsymbol{s}_{\mathrm{f}}=s_{\mathrm{f}} \boldsymbol{e}_{3}$, and $\boldsymbol{\omega}_{\mathrm{E}}=\omega_{\mathrm{E}} \boldsymbol{e}_{3}$, with $\omega_{\mathrm{E}}>0$. With these conventions, and with (10.1), the relevant formulae for the renormalization analysis are the following.

Beside the electric monopole moment (i.e. the charge $-e$ ), the magnetic dipole moment of the empirical electron is the second electromagnetic characteristic measurable in a classical manner. The magnetic moment three-vector $\boldsymbol{\mu}$ given in (9.6) (and re-installing a factor $1 / c$ ) evaluates to

$$
\begin{aligned}
\boldsymbol{\mu} & =-\frac{e}{2 c} \frac{1}{4 \pi R^{2}} \int_{\mathbb{R}^{3}}\left(|\boldsymbol{x}|^{2} \mathrm{Id}-\boldsymbol{x} \otimes \boldsymbol{x}\right) \delta(|\boldsymbol{x}|-R) \mathrm{d}^{3} x \cdot \boldsymbol{\omega}_{\mathrm{E}} \\
& =-\frac{1}{3} \frac{e}{c} R^{2} \omega_{\mathrm{E}} \boldsymbol{e}_{3} .
\end{aligned}
$$

Since it points in the $\boldsymbol{e}_{3}$ direction as well, we will write $\boldsymbol{\mu}=\mu \boldsymbol{e}_{3}$, with $\mu$ read off from (10.4).

The mass $m$ of the renormalized particle is defined by

$$
m c^{2}=W_{\mathrm{b}}+W_{\mathrm{f}} .
$$

Here, $W_{\mathrm{b}}$ is the gyrational energy of the bare particle, defined earlier in (4.2) and evaluated for general spherical $f_{\mathrm{m}}$ in (4.3). Re-installing a factor $c^{2}$ and inserting now (10.1) for $f_{\mathrm{m}}$ in (4.3), we obtain

$$
\begin{aligned}
W_{\mathrm{b}} & =m_{\mathrm{b}} c^{2} \frac{1}{4 \pi R^{2}} \int_{\mathbb{R}^{3}} \frac{1}{\sqrt{1-\left|\boldsymbol{\omega}_{\mathrm{E}} \times \boldsymbol{x}\right|^{2} / c^{2}}} \delta(|\boldsymbol{x}|-R) \mathrm{d}^{3} x \\
& =m_{\mathrm{b}} c^{2} \frac{c}{\omega_{\mathrm{E}} R} \operatorname{artanh}\left(\frac{\omega_{\mathrm{E}} R}{c}\right) .
\end{aligned}
$$

Furthermore, $W_{\mathrm{f}}$ is the electromagnetic field energy of the gyrating charged particle,

$$
\begin{aligned}
W_{\mathrm{f}} & =\frac{1}{8 \pi} \int_{\mathbb{R}^{3}}\left(\left|\mathcal{E}_{s t}(\boldsymbol{x})\right|^{2}+\left|\mathcal{B}_{s t}(\boldsymbol{x})\right|^{2}\right) \mathrm{d}^{3} x \\
& =\frac{1}{2} \frac{e^{2}}{R}\left(1+\frac{2}{9} \frac{\omega_{\mathrm{E}}^{2} R^{2}}{c^{2}}\right) .
\end{aligned}
$$


Our system of equations for the stationary renormalization flow is given by

$$
\begin{aligned}
m_{\mathrm{e}} & =m\left(R, \omega_{\mathrm{E}}, m_{\mathrm{b}}\right), \\
\mu_{\mathrm{e}} & =|\mu|\left(R, \omega_{\mathrm{E}}\right),
\end{aligned}
$$

with the function $m\left(R, \omega_{\mathrm{E}}, m_{\mathrm{b}}\right)$ given through (10.5), (10.7), (10.9), and with the function $|\mu|\left(R, \omega_{\mathrm{E}}\right)$ given through (10.4). Explicitly, we have

$$
\begin{aligned}
m_{\mathrm{e}} & =m_{\mathrm{b}} \frac{c}{\omega_{\mathrm{E}} R} \operatorname{artanh}\left(\frac{\omega_{\mathrm{E}} R}{c}\right)+\frac{1}{2} \frac{e^{2}}{c^{2}} \frac{1}{R}\left(1+\frac{2}{9} \frac{\omega_{\mathrm{E}}^{2} R^{2}}{c^{2}}\right), \\
\mu_{\mathrm{e}} & =\frac{1}{3} \frac{e}{c} R^{2} \omega_{\mathrm{E}} .
\end{aligned}
$$

These two coupled equations determine a curve in $\left(R, \omega_{\mathrm{E}}, m_{\mathrm{b}}\right)$-space for which we will study the limit $m_{\mathrm{b}} \rightarrow 0$.

Equation $(10.13)$ can immediately be solved for $\omega_{\mathrm{E}}$ in terms of the radius $R$,

$$
\omega_{\mathrm{E}}=3 \frac{\mu_{\mathrm{e}} c}{e} \frac{1}{R^{2}} .
$$

Inserted into (10.12), this gives

$$
m_{\mathrm{e}}=m_{\mathrm{b}} R \frac{1}{3} \frac{e}{\mu_{\mathrm{e}}} \operatorname{artanh}\left(3 \frac{\mu_{\mathrm{e}}}{e} \frac{1}{R}\right)+\frac{1}{2} \frac{e^{2}}{c^{2}} \frac{1}{R}\left(1+2 \frac{\mu_{\mathrm{e}}^{2}}{e^{2}} \frac{1}{R^{2}}\right),
$$

which rewrites into a formula for the bare rest mass as function of $R$,

$$
m_{\mathrm{b}}=m_{\mathrm{e}} 3 \frac{\mu_{\mathrm{e}}}{e} \frac{1}{R} \frac{1-\frac{1}{2} \frac{e^{2}}{m_{\mathrm{e}} c^{2}} \frac{1}{R}\left(1+2 \frac{\mu_{\mathrm{e}}^{2}}{e^{2}} \frac{1}{R^{2}}\right)}{\operatorname{artanh}\left(3 \frac{\mu_{\mathrm{e}}}{e} \frac{1}{R}\right)} .
$$

We are interested in the inverse function $R\left(m_{\mathrm{b}}\right)$. In particular, we want to know whether $\lim _{m_{\mathrm{b}} \rightarrow 0} R$ can be taken without encountering superluminal equatorial rotation speeds.

We readily read off from (10.15) that the right side is well-defined only on the $R$ interval $\left(3 \mu_{\mathrm{e}} / e, \infty\right)$. From (10.13) and (10.19) it furthermore follows that $\left|\omega_{\mathrm{E}}\right| R<c$ for $R \in\left(3 \mu_{\mathrm{e}} / e, \infty\right)$, viz., no superluminal rotation speeds occur for $R$ in the domain of definition for the r.h.s. of (10.15). Furthermore, $m_{\mathrm{b}}(R)$ is a monotonically increasing function, so that the limit $R \searrow 3 \mu_{\mathrm{e}} / e$ for $m_{\mathrm{b}}$ will answer the question whether one can take the limit $m_{\mathrm{b}} \rightarrow 0$ for $R$. A simple inspection of (10.15) reveals that $m_{\mathrm{b}} \rightarrow m_{\mathrm{e}}$ as $R \rightarrow \infty$ and that $m_{\mathrm{b}} \rightarrow 0$ as $R \rightarrow 3 \mu_{\mathrm{e}} / e$. Hence, by monotonicity there is a unique limit of vanishing bare rest mass for the radius of the renormalized particle, given by

$$
\lim _{m_{\mathrm{b}} \rightarrow 0} R=3 \frac{\mu_{\mathrm{e}}}{e}
$$

It also follows from our discussion that the equatorial rotation speed in this limit is precisely equal to the speed of light $c$. 
The renormalization flow analysis reveals that the Lorentz program of a "purely electromagnetic electron" can be completed successfully, in the stationary setting at least. The resolution of the puzzlement is that beside the static electromagnetic field energy contributions considered by Lorentz, the mass of the properly renormalized purely electromagnetic particle consists of an additional contribution which we call photonic, for it is the mass of an object without rest mass which rotates with equatorial speed equal to the speed of light. This contribution was missed by Lorentz who calculated without bare rest mass from the outset.

It is instructive now to make use of the empirical relation for the electron magnetic moment, $\mu_{\mathrm{e}}=(1+a) \mu_{\mathrm{B}}$, where

$$
\mu_{\mathrm{B}}=\frac{1}{2} \frac{\hbar e}{m_{\mathrm{e}} c}
$$

is the Bohr magneton and $a=0.001159652 \ldots$ the anomaly factor (see, e.g., [44]). Up to corrections of $O(a)$, equation (10.16) then reveals that the radius of the renormalized purely electromagnetic electron is

$$
3 \frac{\mu_{\mathrm{e}}}{e}=\frac{3}{2} R_{\mathrm{C}},
$$

where

$$
R_{\mathrm{C}}=\frac{\hbar}{m_{\mathrm{e}} c}
$$

is the electron's Compton length. This result is quite remarkable in many respects. It shows that our Lorentz-covariant LED in fact contains the correct ultraviolet cutoff length where heuristic discussions traditionally place the limit of applicability of a classical field theory, viz. at the order of the electron's Compton length $R_{\mathrm{C}}$, rather than at the (lamentably so-called) classical electron radius, given by

$$
R_{\mathrm{Cl}}=\frac{e^{2}}{m_{\mathrm{e}} c^{2}},
$$

which gives the size of a 'purely electrostatic classical electron.' Recalling Sommerfeld's fine structure constant, given by

$$
\alpha=\frac{e^{2}}{\hbar c} \approx \frac{1}{137.036},
$$

we have the relation

$$
R_{\mathrm{Cl}}=\alpha R_{\mathrm{C}}
$$

so that the true Lorentz electron is actually about $(3 / 2 \alpha) \approx 200$ times larger in diameter than predicted by a purely electrostatic calculation.

We conclude this subsection with a discussion of the total spin, given by

$$
s=s_{\mathrm{b}}+s_{\mathrm{f}}
$$


Here, $\boldsymbol{s}_{\mathrm{b}}$ is the spin three-vector of the bare particle, which for $m_{\mathrm{b}}>0$ evaluates to

$$
\begin{aligned}
\boldsymbol{s}_{\mathrm{b}} & =m_{\mathrm{b}} \frac{1}{4 \pi R^{2}} \int_{\mathbb{R}^{3}} \frac{\boldsymbol{x} \times\left(\boldsymbol{\omega}_{\mathrm{E}} \times \boldsymbol{x}\right)}{\sqrt{1-\left|\boldsymbol{\omega}_{\mathrm{E}} \times \boldsymbol{x}\right|^{2} / c^{2}}} \delta(|\boldsymbol{x}|-R) \mathrm{d}^{3} x \\
& =m_{\mathrm{b}} c R\left(\frac{1+c^{2} / \omega_{\mathrm{E}}^{2} R^{2}}{2} \operatorname{artanh}\left(\frac{\omega_{\mathrm{E}} R}{c}\right)-\frac{c}{2 \omega_{\mathrm{E}} R}\right) \boldsymbol{e}_{3},
\end{aligned}
$$

which, by inserting (10.13) into (10.25), with (10.17) for $\mu_{\mathrm{e}}$, becomes

$$
s_{\mathrm{b}}=\hbar \frac{m_{\mathrm{b}}}{m_{\mathrm{e}}} \frac{R}{R_{\mathrm{C}}}\left(\frac{1+\left(2 R / 3 R_{\mathrm{C}}\right)^{2}}{2} \operatorname{artanh}\left(\frac{3}{2} \frac{R_{\mathrm{C}}}{R}\right)-\frac{1}{3} \frac{R}{R_{\mathrm{C}}}\right) .
$$

Using now (10.15), we get

$$
s_{\mathrm{b}}=\frac{3}{2} \hbar \frac{1+\left(2 R / 3 R_{\mathrm{C}}\right)^{2}}{2}\left(1-\frac{1}{2} \frac{R_{C l}}{R}\left(1+\frac{1}{2} \frac{R_{\mathrm{C}}^{2}}{R^{2}}\right)\right)-\hbar \frac{1}{3} \frac{R^{2}}{R_{\mathrm{C}}^{2}} \frac{m_{\mathrm{b}}}{m_{\mathrm{e}}} .
$$

The second spin term, $\boldsymbol{s}_{\mathrm{f}}$, is the stationary field spin three-vector associated with the rotating charged particle, which evaluates to

$$
\begin{aligned}
\boldsymbol{s}_{\mathrm{f}} & =-\frac{e}{c} \frac{1}{4 \pi R^{2}} \int_{\mathbb{R}^{3}} \boldsymbol{x} \times \mathcal{A}_{s t}(\boldsymbol{x}) \delta(|\boldsymbol{x}|-R) \mathrm{d}^{3} x \\
& =\frac{1}{4 \pi c} \int_{\mathbb{R}^{3}} \boldsymbol{x} \times\left(\mathcal{E}_{s t}(\boldsymbol{x}) \times \boldsymbol{\mathcal { B }}_{s t}(\boldsymbol{x})\right) \mathrm{d}^{3} x \\
& =\frac{2}{9} \frac{e^{2}}{c^{2}} \omega_{\mathrm{E}} R \boldsymbol{e}_{3} .
\end{aligned}
$$

We write $\boldsymbol{s}=\left(s_{\mathrm{b}}+s_{\mathrm{f}}\right) \boldsymbol{e}_{3}$, with $s_{\mathrm{b}}$ and $s_{\mathrm{f}}$ given in (10.27) and (10.30).

We consider the spin coefficients $s_{\mathrm{b}}$ and $s_{\mathrm{f}}$ as function of $R$. Letting $R \rightarrow 1.5 R_{\mathrm{C}}$, i.e. taking $m_{\mathrm{b}} \rightarrow 0$, for the bare spin we get,

$$
\lim _{m_{\mathrm{b}} \rightarrow 0} s_{\mathrm{b}}=\frac{3}{2} \hbar\left(1-\frac{11}{27} \alpha\right)
$$

while for the field spin, recalling that $\omega_{\mathrm{E}} R \rightarrow c$ as $m_{\mathrm{b}} \rightarrow 0$, we immediately obtain from (10.30) that

$$
\lim _{m_{\mathrm{b}} \rightarrow 0} s_{\mathrm{f}}=\frac{2}{9} \frac{e^{2}}{c}=\frac{2}{9} \alpha \hbar .
$$

Finally, adding $s_{\mathrm{f}}$ and $s_{\mathrm{b}}$ and taking the limit equals the sum of the individual limits, whence the renormalized limit spin magnitude is given by

$$
s_{\text {ren }} \stackrel{\text { def }}{=} \lim _{m_{\mathrm{b}} \rightarrow 0} s=\frac{3}{2} \hbar\left(1-\frac{7}{27} \alpha\right) .
$$

Put differently, from (10.33) we read off that the spin magnitude $s_{\text {ren }}$ of the renormalized electron satisfies

$$
s_{\text {ren }} \approx \frac{3}{2} \hbar
$$


to high precision, with relative corrections of order $\alpha$, compared to our naïve expectation $s \approx \hbar$, and compared to $s \approx \hbar / 2$ in a quantum mechanical framework.

Curiously, furthermore, a closer look at the corrections reveals that in our LED an expansion of the $g$-factor in the Landé relation (10.2) in terms of powers of $\alpha$ holds. We set $g_{0} \stackrel{\text { def }}{=} 2 / 3$, then

$$
g_{\mathrm{LED}}=g_{0}\left(1+\frac{7}{27} \alpha+\left(\frac{7}{27}\right)^{2} \alpha^{2}+O\left(\alpha^{3}\right)\right)
$$

which is surprisingly similar to the familiar expansion in QED 44, 11,

$$
g_{\mathrm{QED}}=g_{\mathrm{QM}}\left(1+\frac{1}{2 \pi} \alpha-\frac{0.328 \ldots}{\pi^{2}} \alpha^{2}+O\left(\alpha^{3}\right)\right) \text {. }
$$

\subsection{The limit $m_{\mathbf{b}} \rightarrow 0^{+}$for the dynamical electron}

We finally come the dynamical renormalization problem. In contrast to the stationary problem, no rigorous results concerning the existence of this limit are available yet. However, it is of interest to inquire into the features expected for a renormalized dynamical limit of vanishing bare rest mass of massive LED.

First of all, the matching of empirical electron data in the dynamical renormalization problem with scattering states has to be carried out in the asymptotic past (or future), for we want to "send in" the particle with the correct physical characteristic particle data. The incoming-data matching in the asymptotic past will fix $R$ and $m_{\mathrm{b}}$ for the rest of the motion, since as parameters of the model these do not vary during the motion.

This matching in the asymptotic past will also fix the value of $\left|\boldsymbol{\omega}_{\mathrm{E}}\right|$ in the asymptotic future, which is due to the soliton dynamics of the renormalized particle. During the motion, of course, $\left|\boldsymbol{\omega}_{\mathrm{E}}\right|$ will in general vary. However, our results on the scattering with a non-moving particle indicate that $\left|\boldsymbol{\omega}_{\mathrm{E}}\right|$ approaches its asymptotic value extremely fast. Extrapolating from this observation, we expect that the soliton dynamics of the particle in massive LED in fact will try to keep the angular frequency $\left|\boldsymbol{\omega}_{\mathrm{E}}\right|$ of the particle as close as possible to the asymptotic value in the free motions. By the same token the gyrational mass (hence, also the renormalized mass, which is a small perturbation of the former in the stationary state) of the particle should remain (in the instantaneous rest frame) very close to the empirical mass during the motion.

An important conclusion can then be drawn if one can show that the gyrational bare mass of the particle with $m_{\mathrm{b}}>0$ stays uniformly close to the empirical mass as $m_{\mathrm{b}} \rightarrow 0$ for an $m_{\mathrm{b}}$-indexed sequence of motions. Namely, in that case the equatorial gyration speed of the renormalized purely electromagnetic particle must be locked at the speed of light during the whole motion! For assuming to the contrary that the gyrational speed would remain bounded away from the speed of light for at least a finite fraction of the evolution (of course still close to it in the uniform sense stipulated), it would then follow that the bare gyrational mass converges to zero, while the electromagnetic mass would contribute at best an amount of order $\alpha$ of the empirical mass. This is in contradiction to its hypothesized uniform proximity to the empirical mass. 
We infer that in the limit $m_{\mathrm{b}} \rightarrow 0$ the bare gyrational mass $\mathcal{M}_{\mathrm{b}}$ is independent of $\boldsymbol{\omega}_{\mathrm{E}}$, hence a constant of the motion identical to what we have called the photonic mass of the stationary particle; explicitly,

$$
m_{\mathrm{ph}}=m_{\mathrm{e}}\left(1-\frac{11}{27} \alpha\right) .
$$

By the same reasoning, in the limit of vanishing bare rest mass the bare spin vector becomes the photonic spin, a four-vector with only two independent variables. It's dual tensor is given by

$$
\mathbf{S}_{\mathrm{ph}}=\frac{3}{2} \hbar\left(1-\frac{11}{27} \alpha\right) \boldsymbol{\Sigma},
$$

where $\boldsymbol{\Sigma}$ is anti-symmetric, of space-space type, has norm 1, satisfying $\boldsymbol{\Sigma} \cdot \boldsymbol{u}=0$. The inertial gyro-frequency $\Omega_{\mathrm{E}}$ and the spin tensor $\boldsymbol{\Sigma}$ are related by a simple proportionality,

$$
\Omega_{\mathrm{E}}=\frac{m_{e} c^{2}}{\hbar} \Sigma
$$

emphasizing the characteristic Euler angular frequency $m_{e} c^{2} / \hbar$ explicitly. Similarly, $\boldsymbol{\Omega}_{\mathrm{E}}=$ $\kappa \mathbf{S}_{\mathrm{ph}}$, with

$$
\kappa=\frac{m_{e} c^{2}}{\hbar^{2}} \frac{1}{1-\frac{11}{27} \alpha} .
$$

After these preparations, we can write down the putative dynamical equations of renormalized LED. The world-line equation of renormalized LED reads

$$
\frac{\mathrm{d}}{\mathrm{d} \tau} \boldsymbol{p}=\int_{\mathbb{R}^{1,3}} \mathbf{F}(\boldsymbol{q}+\mathbf{x}) \cdot\left(\boldsymbol{u}-\left(\boldsymbol{\Omega}_{\mathrm{FW}}+\kappa \mathbf{S}_{\mathrm{ph}}\right) \cdot \mathbf{x}\right) f_{\mathrm{e}}(\|\mathbf{x}\|) \delta(\boldsymbol{u} \cdot \mathbf{x}) \mathrm{d}^{4} \mathbf{x},
$$

where

$$
\boldsymbol{p}(\tau)=\mathbf{M}_{\mathrm{ren}}(\tau) \cdot \mathbf{u}(\tau)
$$

is the energy-momentum four-vector of the renormalized purely electromagnetic electron, with $\mathbf{M}_{\text {ren }}$ given by

$$
\mathbf{M}_{\mathrm{ren}}=m_{\mathrm{ph}} \mathbf{g}-\int_{\mathbb{R}^{1,3}}\left[\mathbf{x} \otimes \mathbf{x},\left[\mathbf{F}(\boldsymbol{q}+\mathbf{x}), \kappa \mathbf{S}_{\mathrm{ph}}\right]_{+}\right]_{+} f_{\mathrm{e}}(\|\mathbf{x}\|) \delta(\mathbf{u} \cdot \mathbf{x}) \mathrm{d}^{4} \mathbf{x}
$$

now the renormalization limit of the tensor mass $\mathbf{M}$. The gyrograph equation of renormalized LED reads

$$
\begin{aligned}
& \frac{\mathrm{d}}{\mathrm{d} \tau} \mathbf{S}_{\mathrm{ph}}+\left[\boldsymbol{\Omega}_{\mathrm{FW}}, \mathbf{S}_{\mathrm{ph}}\right]_{-}= \\
& \quad \int_{\mathbb{R}^{1,3}} \boldsymbol{x} \wedge(\mathbf{g}+\mathbf{u} \otimes \boldsymbol{u}) \cdot \mathbf{F}(\boldsymbol{q}+\boldsymbol{x}) \cdot\left(\boldsymbol{u}-\left(\boldsymbol{\Omega}_{\mathrm{FW}}+\kappa \mathbf{S}_{\mathrm{ph}}\right) \cdot \boldsymbol{x}\right) f_{\mathrm{e}}(\|\boldsymbol{x}\|) \delta(\boldsymbol{u} \cdot \boldsymbol{x}) \mathrm{d}^{4} \boldsymbol{x} .
\end{aligned}
$$


The Maxwell-Lorentz equations for renormalized LED have their familiar structure, but the charge-current density is now given by

$$
\boldsymbol{J}(\mathbf{x})=\int_{-\infty}^{+\infty}\left(\boldsymbol{u}-\left(\boldsymbol{\Omega}_{\mathrm{FW}}+\kappa \mathbf{S}_{\mathrm{ph}}\right) \cdot(\mathbf{x}-\boldsymbol{q})\right) f_{\mathrm{e}}(\|\mathbf{x}-\boldsymbol{q}\|) \delta(\mathbf{u} \cdot(\mathbf{x}-\boldsymbol{q})) \mathrm{d} \tau .
$$

The state in renormalized LED differs from that of massive LED in that only the orientation but not the norm of $\mathbf{S}_{\mathrm{ph}}$ is a dynamical degree of freedom. This imposes some consistency conditions also on the initial conditions. We remark that (10.44) does not automatically preserve $\left\|\mathbf{S}_{\mathrm{ph}}\right\|$ for an arbitrary electromagnetic tensor field $\mathbf{F}$ and world-line $\tau \mapsto \boldsymbol{q}$. However, it is only mandatory that the norm of $\mathbf{S}_{\mathrm{ph}}$ be conserved for consistent joint solutions of the world-line, gyrograph, and field equations; the situation is similar to the one related to the conservation of $\|\mathbf{u}\|^{2}$ by the world-line equation.

\section{Summary and Outlook}

In the previous sections we have constructed the first viable relativistic Lorentz electrodynamics with positive bare rest mass (massive LED). We studied its renormalization flow limit to vanishing bare rest mass (renormalized LED). This modern completion of Lorentz' program of a purely electromagnetic theory avoids the superluminal pitfalls of Lorentz' purely electromagnetic calculations. In the limit we obtain a particle with correct empirical mass, charge and magnetic moment, though with spin $3 \hbar / 2$. Although the bare rest mass vanishes, the particle retains a "photonic mass" resulting from luminal equatorial motions, which actually accounts for most of the particle's mass while the traditional electromagnetic self-energies contribute merely a correction of order $\alpha$. The size of the limit particle is of the order of the electron's Compton length, about 200 classical electron radii.

The finite size of this ultraviolet cutoff in the 'purely electromagnetic' limit endows our LED with a mildly a-causal appearance. However, the ultraviolet cutoff is itself an individual but non-local event which cannot be subdivided into a collection of individual point events. The cutoff moves as a non-local unit in a Lorentz-covariant way, in fact accelerated in a causal manner - in the sense that fields outside the cutoff event do not influence its motion - by the field integrated over the cutoff at any given instant of its proper-time. It is perhaps surprising that such an instantaneous, non-local action is compatible with Lorentz covariance and causality, yet it is.

The existence of this physically viable properly renormalized LED reinforces some hopes that LED may ultimately have some bearing on the quest for a consistent QED, with LED or some improved version thereof $\left(\mathrm{LED}^{+}\right.$say) as its (semi-)classical limit. Recall that the perturbative series for QED [45] is most likely divergent [46] and hence does not give a mathematically acceptable definition of QED. Many experts nowadays are inclined to believe that the constructive quantum field theory approach to QED [11] will not lead to a mathematically consistent theory either [47], though the failure of this approach to QED has not yet been conclusively demonstrated [48]. There is also a strong anticipation that a physically correct and mathematically well-defined relativistic quantum theory must involve also the strong and weak nuclear interactions, as well as gravity, and all this supersymmetrically [49, 50, 51]. It should however not be necessary to unify physics at the Planck scale 
in order to obtain a consistent working theory for electromagnetic phenomena above the electron's Compton scale. Inspired by Kramer's program of 'non-relativistic QED' [52, 53], which aims at the quantization of LED's semi-relativistic predecessor (see Appendix A.3), and which is expected to describe the low energy limit of QED [53], one may want to investigate the possibility of deforming our LED into a 'quantized LED', perhaps along the ideas of Kontsevich [54], and it would be interesting to see whether that would teach us something about QED proper.

Such fundamental hopes aside, our LED is interesting in itself as a nonlinear relativistic microscopic theory which couples particle and field degrees of freedom in a self-consistent manner. While the rigorous control of its semi-relativistic predecessor has made significant advances in recent years due to the efforts of Spohn and collaborators and others (discussed in the Appendix), the extension of this rigorous control to our relativistic LED has just started [33]. The most surprising new dynamical challenge is the need to construct a compatible foliation of spacetime along the evolution of the state in LED by also solving the vacuum Einstein equations as a free-boundary problem coupled to the evolution of the state. Unexpectedly, massive and renormalized LED may therefore serve as playground for the harder problem of black hole dynamics in general relativity.

Among the future projects, one is certainly the rigorous extraction of effective equations of motion for the particle in the adiabatic regime, where to lowest order one expects equations of the type discussed in [55, 56], with radiation reaction-corrections showing in the next order of approximation.

Another interesting project is the many particle problem, and in particular the rigorous microscopic foundation of relativistic Vlasov-Maxwell theory [57, 58, 59, 60], which so far could not even be attempted for lack of a consistent microscopic theory of electromagnetic particles and fields.

\section{A Appendices}

Appendix A.1 contains the more technical derivations of the Euler-Lagrange equations for our massive LED, Appendix A.2 discusses the singular point mass limit in which our massive LED reduces to Nodvik's massive LED, and Appendix A.3 provides supplementary material about the semi-relativistic LED.

\section{A.1 The Euler-Lagrange equations of massive LED}

In this subsection, we first collect various formulas needed to carry out the variations of the action functional. Next we apply these formulas to derive the Euler-Lagrange equations for the action principle.

\section{A.1.1 Independent variation of the world-line}

To a given world-line $\tau \mapsto \boldsymbol{q}(\tau)$ we may add a small perturbation $\tau \mapsto \delta \boldsymbol{q}(\tau)$ to get a deformed particle world-line $\check{\boldsymbol{q}}(\tau)=(\boldsymbol{q}+\delta \boldsymbol{q})(\tau)$. Here, $\tau$ is the proper-time of the unperturbed worldline. The proper-time along the perturbed world-line at the point $\check{\mathbf{q}}(\tau)$ will in general not 
coincide with the value $\tau$, i.e. the proper-time of the unperturbed world-line. To first order, the difference in the proper-times along the deformed and original world-line reads

$$
\delta(\mathrm{d} \tau)=-\mathbf{u} \cdot \delta \stackrel{\circ}{q} \mathrm{~d} \tau
$$

Accordingly, the derivative of $\delta \boldsymbol{q}$ with respect to $\tau$, viz. $\delta \dot{q}$, is generally not the first-order variation in the four-velocity, $\delta \mathbf{u}$, which is defined by taking the derivative of $\check{\boldsymbol{q}}$ w.r.t. its proper-time, then computing the difference $\check{\mathbf{u}}-\mathbf{u}$ to first order. As a function of $\tau$, the first-order variation in the four-velocity is given by the space projection (w.r.t. $\mathbf{u}$ ) of $\delta \mathfrak{q}$,

$$
\delta \mathbf{u}=(\mathbf{g}+\mathbf{u} \otimes \mathbf{u}) \cdot \stackrel{\circ}{\delta} \mathbf{q}
$$

where $\mathbf{g}$ is the metric tensor of Minkowski space, defined in (2.12). Taking the inner product of (A.2) with $\mathbf{u}$, and recalling that $\mathbf{g}$ acts as identity on four-vectors, we find that $\mathbf{u} \cdot \delta \mathbf{u}=0$, as required by $(\boldsymbol{u}+\delta \mathbf{u}) \cdot(\mathbf{u}+\delta \mathbf{u})=-1$ in first order. The variation of $\boldsymbol{\Omega}_{\mathrm{FW}}$ under an independent change $\delta \boldsymbol{q}$, the gyrograph being kept fixed, is given by 15 :

$$
\delta \boldsymbol{\Omega}_{\mathrm{FW}}=-\mathbf{u} \wedge \stackrel{\circ}{\delta \boldsymbol{q}}+\boldsymbol{\Omega}_{\mathrm{FW}} \mathbf{u} \cdot \stackrel{\circ}{\delta \boldsymbol{q}} .
$$

Defining quasi-coordinates

$$
\delta \boldsymbol{\vartheta} \stackrel{\text { def }}{=}-\mathbf{u} \wedge \delta \mathbf{u}
$$

equation (A.3) can be rewritten into

$$
\delta \boldsymbol{\Omega}_{\mathrm{FW}}=\frac{\mathrm{d}}{\mathrm{d} \tau} \delta \boldsymbol{\vartheta}+\left[\boldsymbol{\Omega}_{\mathrm{FW}}, \delta \boldsymbol{\vartheta}\right]_{-}+\boldsymbol{\Omega}_{\mathrm{FW}} \boldsymbol{u} \cdot \stackrel{\circ}{\boldsymbol{q}},
$$

which proves convenient later on.

Moreover, although the gyrograph is fixed during an independent variation of the worldline, the Fermi-Walker frame changes, and with it the tensor of the Euler gyration, for

the gyrograph is defined w.r.t. the Fermi-Walker frame. The variation in $\Omega_{\mathrm{E}}$ under an independent change $\delta \boldsymbol{q}$ is given by [15]

$$
\delta \boldsymbol{\Omega}_{\mathrm{E}}=-\mathbf{u} \wedge\left(\boldsymbol{\Omega}_{\mathrm{E}} \cdot \delta \mathbf{u}\right)+\boldsymbol{\Omega}_{\mathrm{E}} \mathbf{u} \cdot \delta \stackrel{\circ}{\boldsymbol{q}}
$$

whence

$$
\delta \boldsymbol{\Omega}=-\mathbf{u} \wedge(\boldsymbol{\Omega} \cdot \delta \mathbf{u})-\mathbf{u} \wedge \stackrel{\circ}{\delta} \boldsymbol{q}+\boldsymbol{\Omega} \mathbf{u} \cdot \delta \stackrel{\circ}{q}
$$

\section{A.1.2 Independent variation of the gyrograph}

The orientation of the set of three spacelike vectors $\left\{\tilde{\boldsymbol{e}}_{1}, \tilde{\mathbf{e}}_{2}, \tilde{\boldsymbol{e}}_{3}\right\}$ relative to the spacelike vectors $\left\{\overline{\boldsymbol{e}}_{1}, \overline{\boldsymbol{e}}_{2}, \overline{\boldsymbol{e}}_{3}\right\}$ can be specified by Euler angles $\theta, \phi, \psi$. Independent changes $\delta \theta, \delta \phi$, 
$\delta \psi$ of the particle's history of Euler angles, i.e. keeping its world-line fixed, give rise to the variation 15

$$
\delta \boldsymbol{\Omega}_{\mathrm{E}}=\stackrel{\circ}{\delta \boldsymbol{\theta}}+[\boldsymbol{\Omega}, \delta \boldsymbol{\theta}]_{-},
$$

where the anti-symmetric tensor $\delta \boldsymbol{\theta}$ is given by

$$
\delta \boldsymbol{\theta}=\sum_{0 \leqslant \mu, \nu \leqslant 3} \delta \bar{\theta}^{\mu \nu} \overline{\mathbf{e}}_{\mu} \otimes \overline{\mathbf{e}}_{\nu}
$$

with $\left\{\overline{\boldsymbol{e}}_{\mu}\right\}$ the tetrad of the frame $\mathcal{F}_{\mathrm{FW}}$ and $\delta \bar{\theta}_{\mu}{ }^{\nu}$ given by

$$
\left\{\begin{array}{l}
\delta \bar{\theta}_{1}{ }^{2}=\delta \phi+\cos \theta \delta \psi \\
\delta \bar{\theta}_{2}{ }^{3}=\cos \phi \delta \theta+\sin \theta \sin \phi \delta \psi \\
\delta \bar{\theta}_{3}{ }^{1}=\sin \phi \delta \theta-\sin \theta \cos \phi \delta \psi \\
\delta \bar{\theta}_{0}{ }^{i}=0 \quad \text { for } i=1,2,3
\end{array}\right.
$$

cf. [15] for details.

\section{A.1.3 Euler-Lagrange equations for the world-line}

We first turn to the action of the bare particle, which we rewrite as

$$
\mathcal{A}_{\text {bare }}=\int_{\tau_{1}}^{\tau_{2}} L_{\mathrm{b}}\left(\boldsymbol{\Omega}_{\mathrm{E}}, \boldsymbol{u}\right) \mathrm{d} \tau
$$

where

$$
L_{\mathrm{b}}\left(\boldsymbol{\Omega}_{\mathrm{E}}, \boldsymbol{u}\right) \stackrel{\text { def }}{=}-\int_{\Xi_{q}} \sqrt{1-\left\|\boldsymbol{\Omega}_{\mathrm{E}} \cdot \boldsymbol{x}\right\|^{2}} f_{\mathrm{m}}(\|\boldsymbol{x}\|) \delta(\boldsymbol{u} \cdot \mathbf{x}) \mathrm{d}^{4} \boldsymbol{x}
$$

is the Lagrangian for the bare particle. We first define the volume $\Xi$ bounded by two space slices defined by $\mathbf{u}(\tau) \cdot(\mathbf{x}-\boldsymbol{q}(\tau))=0$ at $\tau=\tau_{1}$ and $\tau=\tau_{2}$. Its translation by $\boldsymbol{q}$ is denoted by $\Xi_{\boldsymbol{q}}=\{\mathbf{x}-\boldsymbol{q}(\tau) ; \mathbf{x} \in \Xi\}$. Under an independent variation $\boldsymbol{q} \mapsto \boldsymbol{q}+\delta \boldsymbol{q}$ the resulting first variation of $\mathcal{A}_{\text {bare }}$ reads

$$
\delta \mathcal{A}_{\text {bare }}=\int_{\tau_{1}}^{\tau_{2}}\left(\frac{1}{2} \operatorname{Tr}\left(\frac{\delta L_{\mathrm{b}}}{\delta \boldsymbol{\Omega}_{\mathrm{E}}} \cdot \delta \boldsymbol{\Omega}_{\mathrm{E}}\right)+\frac{\delta L_{\mathrm{b}}}{\delta \boldsymbol{u}} \cdot \delta \mathbf{u}-L_{\mathrm{b}} \boldsymbol{u} \cdot \delta \stackrel{\circ}{\mathbf{q}}\right) \mathrm{d} \tau,
$$

where the last term under the integral results from the change in proper time along the perturbed world-line, see equation (A.1). As for the variation of $L_{\mathrm{b}}$ w.r.t. $\boldsymbol{u}$, we get

$$
\begin{aligned}
\frac{\delta L_{\mathrm{b}}}{\delta \boldsymbol{u}} & =-\int_{\Xi_{q}} \sqrt{1-\left\|\boldsymbol{\Omega}_{\mathrm{E}} \cdot \mathbf{x}\right\|^{2}} f_{\mathrm{m}}(\|\mathbf{x}\|) \partial_{\boldsymbol{u}} \delta(\mathbf{u} \cdot \mathbf{x}) \mathrm{d}^{4} \mathbf{x} \\
& =-\boldsymbol{u} \int_{\Xi_{\boldsymbol{q}}} \sqrt{1-\left\|\boldsymbol{\Omega}_{\mathrm{E}} \cdot \boldsymbol{x}\right\|^{2}} f_{\mathrm{m}}(\|\boldsymbol{x}\|) \delta(\boldsymbol{u} \cdot \mathbf{x}) \mathrm{d}^{4} \boldsymbol{x},
\end{aligned}
$$


where we used

$$
\partial_{\mathbf{u}} \delta(\mathbf{u} \cdot \mathbf{x})=-\mathbf{x}\left(\mathbf{u} \cdot \nabla_{\mathrm{g}}\right) \delta(\mathbf{u} \cdot \mathbf{x})
$$

then integrated by parts, carried out the differentiations, and noticed that terms proportional to $\mathbf{u} \cdot \boldsymbol{x}$ in the integrand vanish upon integration against $\delta(\mathbf{u} \cdot \mathbf{x})$. But with the help of the four-orthogonality of $\boldsymbol{u}$ and $\delta \boldsymbol{u}$ we see that

$$
\frac{\delta L_{\mathrm{b}}}{\delta \mathbf{u}} \cdot \delta \mathbf{u}=0
$$

As for the variation of $L_{\mathrm{b}}$ w.r.t. $\Omega_{\mathrm{E}}$, we find that

$$
\frac{\delta L_{\mathrm{b}}}{\delta \Omega_{\mathrm{E}}}=\mathbf{S}_{\mathrm{b}},
$$

where $\mathbf{S}_{\mathrm{b}}$ is the spin tensor of the bare particle, defined earlier in (6.12). Thus, we have

$$
\delta \mathcal{A}_{\mathrm{bare}}=\int_{\tau_{1}}^{\tau_{2}}\left(\frac{1}{2} \operatorname{Tr}\left(\mathbf{S}_{\mathrm{b}} \cdot \delta \boldsymbol{\Omega}_{\mathrm{E}}\right)-L_{\mathrm{b}} \boldsymbol{u} \cdot \delta \boldsymbol{\circ}\right) \mathrm{d} \tau
$$

Inserting now (A.6) for $\delta \boldsymbol{\Omega}_{\mathrm{E}}$, with (A.2) in place for $\delta \boldsymbol{u}$, then using $\boldsymbol{\Omega}_{\mathrm{E}} \cdot \boldsymbol{u}=\boldsymbol{0}$ once, next integrating by parts terms containing $\delta \boldsymbol{q}$, recalling that $\delta \boldsymbol{q}=0$ at the boundaries of integration, then using again $\boldsymbol{\Omega}_{\mathrm{E}} \cdot \boldsymbol{u}=\boldsymbol{O}$ and also noticing once again that terms proportional to $\mathbf{u} \cdot \boldsymbol{x}$ in the integrand vanish upon integration against $\delta(\mathbf{u} \cdot \mathbf{x})$, we obtain

$$
\delta \mathcal{A}_{\mathrm{bare}}=-\int_{\tau_{1}}^{\tau_{2}} \stackrel{\circ}{\boldsymbol{p}}_{\mathrm{b}}(\tau) \cdot \delta \boldsymbol{q} \mathrm{d} \tau
$$

Here, $\boldsymbol{p}_{\mathrm{b}}$ is the Minkowski momentum four-vector of the bare particle, which for later convenience we write in the form

$$
p_{\mathrm{b}}=\mathrm{M}_{\mathrm{b}} \cdot \mathbf{u}
$$

where

$$
\mathbf{M}_{\mathrm{b}}=\mathcal{M}_{\mathrm{b}} \mathbf{g}
$$

is the diagonal bare mass tensor, with

$$
\mathcal{M}_{\mathrm{b}}\left(\left\|\boldsymbol{w}_{\mathrm{E}}\right\|\right)=\int_{\Xi_{q}} \frac{1}{\sqrt{1-\left\|\Omega_{\mathrm{E}} \cdot \boldsymbol{x}\right\|^{2}}} f_{\mathrm{m}}(\|\mathbf{x}\|) \delta(\boldsymbol{u} \cdot \mathbf{x}) \mathrm{d}^{4} \mathbf{x}
$$

the gyrational bare mass of the particle.

Turning next to the action of field-particle coupling, we rewrite it as

$$
\mathcal{A}_{\text {coupl }}=\int_{\tau_{1}}^{\tau_{2}} L_{\mathrm{c}}\left(\boldsymbol{\Omega}_{\mathrm{E}}, \boldsymbol{q}, \mathbf{u}, \stackrel{\circ}{\mathbf{u}}\right) \mathrm{d} \tau,
$$


where

$$
L_{\mathrm{c}}\left(\boldsymbol{\Omega}_{\mathrm{E}}, \boldsymbol{q}, \mathbf{u}, \stackrel{\circ}{\mathbf{u}}\right)=\int_{\Xi_{q}}(\mathbf{u}-\boldsymbol{\Omega} \cdot \boldsymbol{x}) \cdot \boldsymbol{A}(\boldsymbol{q}+\mathbf{x}) f_{\mathrm{e}}(\|\mathbf{x}\|) \delta(\mathbf{u} \cdot \mathbf{x}) \mathrm{d}^{4} \mathbf{x}
$$

is the Lagrangian for the coupling of the particle to the electromagnetic fields. Since $\Omega_{\mathrm{E}}$ and $\stackrel{\circ}{\mathbf{u}}$ occur in $L_{\mathrm{c}}$ only in the combination $\boldsymbol{\Omega}_{\mathrm{E}}+\stackrel{\circ}{\mathbf{u}} \wedge \mathbf{u}=\boldsymbol{\Omega}$, we can treat $L_{\mathrm{c}}$ as a function of $\boldsymbol{\Omega}, \boldsymbol{q}, \boldsymbol{u}$. Thus, abusing notation a little bit, we write $L_{\mathrm{c}}(\boldsymbol{\Omega}, \boldsymbol{q}, \boldsymbol{u})$ and obtain

$$
\delta \mathcal{A}_{\text {coupl }}=\int_{\tau_{1}}^{\tau_{2}}\left(\frac{1}{2} \operatorname{Tr}\left(\frac{\delta L_{\mathrm{c}}}{\delta \boldsymbol{\Omega}} \cdot \delta \boldsymbol{\Omega}\right)+\frac{\delta L_{\mathrm{c}}}{\delta \boldsymbol{q}} \cdot \delta \boldsymbol{q}+\frac{\delta L_{\mathrm{c}}}{\delta \boldsymbol{u}} \cdot \delta \mathbf{u}-L_{\mathrm{c}} \mathbf{u} \cdot \delta \boldsymbol{q}\right) \mathrm{d} \tau
$$

under independent variation of the world-line. As for the variation of $L_{\mathrm{c}}$ w.r.t. $\boldsymbol{u}$, we have

$$
\begin{aligned}
\frac{\delta L_{\mathrm{c}}}{\delta \mathbf{u}}= & \int_{\Xi_{q}} \boldsymbol{A}(\boldsymbol{q}+\mathbf{x}) f_{\mathrm{e}}(\|\mathbf{x}\|) \delta(\mathbf{u} \cdot \mathbf{x}) \mathrm{d}^{4} \mathbf{x} \\
& +\int_{\Xi_{q}}(\mathbf{u}-\boldsymbol{\Omega} \cdot \mathbf{x}) \cdot \boldsymbol{A}(\boldsymbol{q}+\mathbf{x}) f_{\mathrm{e}}(\|\mathbf{x}\|) \partial_{\mathbf{u}} \delta(\mathbf{u} \cdot \mathbf{x}) \mathrm{d}^{4} \mathbf{x} .
\end{aligned}
$$

Using once again (A.16), then integrating by parts, carrying out the differentiations, and noticing once again that terms proportional to $\boldsymbol{u} \cdot \boldsymbol{x}$ in the integrand vanish upon integration against $\delta(\boldsymbol{u} \cdot \boldsymbol{x})$, we obtain

$$
\begin{aligned}
\frac{\delta L_{\mathrm{c}}}{\delta \boldsymbol{u}}= & \int_{\Xi_{q}} \boldsymbol{A}(\boldsymbol{q}+\mathbf{x}) \delta(\mathbf{u} \cdot \mathbf{x}) f_{\mathrm{e}}(\|\mathbf{x}\|) \mathrm{d}^{4} \mathbf{x} \\
& +\boldsymbol{u} \int_{\Xi_{q}}(\mathbf{u}-\boldsymbol{\Omega} \cdot \mathbf{x}) \cdot \boldsymbol{A}(\boldsymbol{q}+\mathbf{x}) f_{\mathrm{e}}(\|\mathbf{x}\|) \delta(\mathbf{u} \cdot \mathbf{x}) \mathrm{d}^{4} \mathbf{x} \\
& +\int_{\Xi_{q}} \mathbf{x} \stackrel{\circ}{\mathbf{u}} \cdot \boldsymbol{A}(\boldsymbol{q}+\mathbf{x}) f_{\mathrm{e}}(\|\mathbf{x}\|) \delta(\mathbf{u} \cdot \mathbf{x}) \mathrm{d}^{4} \mathbf{x} \\
& +\int_{\Xi_{q}} \mathbf{x}(\mathbf{u}-\boldsymbol{\Omega} \cdot \mathbf{x}) \cdot\left(\mathbf{u} \cdot \nabla_{\mathrm{g}} \boldsymbol{A}(\boldsymbol{q}+\mathbf{x})\right) f_{\mathrm{e}}(\|\mathbf{x}\|) \delta(\mathbf{u} \cdot \mathbf{x}) \mathrm{d}^{4} \mathbf{x}
\end{aligned}
$$

Using the four-orthogonality of $\boldsymbol{u}$ and $\delta \mathbf{u}$, we thus have

$$
\begin{aligned}
\frac{\delta L_{\mathrm{c}}}{\delta \mathbf{u}} \cdot \delta \mathbf{u}= & \int_{\Xi_{q}} \delta \mathbf{u} \cdot \boldsymbol{A}(\boldsymbol{q}+\mathbf{x}) \delta(\mathbf{u} \cdot \mathbf{x}) f_{\mathrm{e}}(\|\mathbf{x}\|) \mathrm{d}^{4} \mathbf{x} \\
& +\int_{\Xi_{q}} \delta \mathbf{u} \cdot \mathbf{x} \stackrel{\circ}{ } \cdot \boldsymbol{A}(\boldsymbol{q}+\mathbf{x}) f_{\mathrm{e}}(\|\mathbf{x}\|) \delta(\mathbf{u} \cdot \mathbf{x}) \mathrm{d}^{4} \boldsymbol{x} \\
& +\int_{\Xi_{q}} \delta \mathbf{u} \cdot \mathbf{x}(\mathbf{u}-\boldsymbol{\Omega} \cdot \mathbf{x}) \cdot\left(\mathbf{u} \cdot \nabla_{\mathrm{g}} \boldsymbol{A}(\boldsymbol{q}+\mathbf{x})\right) f_{\mathrm{e}}(\|\mathbf{x}\|) \delta(\mathbf{u} \cdot \mathbf{x}) \mathrm{d}^{4} \mathbf{x}
\end{aligned}
$$

As for the variation of $L_{\mathrm{c}}$ w.r.t. $\boldsymbol{q}$, we have

$$
\frac{\delta L_{\mathrm{c}}}{\delta \boldsymbol{q}} \cdot \delta \boldsymbol{q}=\int_{\Xi_{q}}(\boldsymbol{u}-\boldsymbol{\Omega} \cdot \boldsymbol{x}) \cdot\left(\frac{\partial \boldsymbol{A}(\boldsymbol{q}+\mathbf{x})}{\partial \boldsymbol{q}} \cdot \delta \boldsymbol{q}\right) f_{\mathrm{e}}(\|\mathbf{x}\|) \delta(\mathbf{u} \cdot \mathbf{x}) \mathrm{d}^{4} \mathbf{x}
$$


while for the variation of $L_{\mathrm{c}}$ w.r.t. $\Omega$ we simply have

$$
\frac{1}{2} \operatorname{Tr}\left(\frac{\delta L_{\mathrm{c}}}{\delta \boldsymbol{\Omega}} \cdot \delta \boldsymbol{\Omega}\right)=-\int_{\Xi_{\boldsymbol{q}}}(\delta \boldsymbol{\Omega} \cdot \mathbf{x}) \cdot \boldsymbol{A}(\boldsymbol{q}+\mathbf{x}) f_{\mathrm{e}}(\|\mathbf{x}\|) \delta(\mathbf{u} \cdot \mathbf{x}) \mathrm{d}^{4} \mathbf{x}
$$

We use (A.7) for the variation of the total gyration tensor $\Omega$ along the independently perturbed particle world-line, and find

$$
\begin{aligned}
& \delta \mathcal{A}_{\text {coupl }}=\int_{\tau_{1}}^{\tau_{2}} \frac{\mathrm{d}}{\mathrm{d} \tau}\left\{-(\mathbf{g}+\mathbf{u} \otimes \mathbf{u}) \cdot \frac{\mathrm{d}}{\mathrm{d} \tau} \int_{\Xi_{\boldsymbol{q}}} \mathbf{x} \mathbf{u} \cdot \boldsymbol{A}(\boldsymbol{q}+\mathbf{x}) f_{\mathrm{e}}(\|\mathbf{x}\|) \delta(\mathbf{u} \cdot \mathbf{x}) \mathrm{d}^{4} \mathbf{x}\right. \\
& +\int_{\Xi_{q}}\left[\boldsymbol{A}(\boldsymbol{q}+\mathbf{x})-(\mathbf{u} \cdot \boldsymbol{A}(\boldsymbol{q}+\mathbf{x})) \boldsymbol{\Omega}_{\mathrm{E}} \cdot \mathbf{x}\right] f_{\mathrm{e}}(\|\mathbf{x}\|) \delta(\boldsymbol{u} \cdot \mathbf{x}) \mathrm{d}^{4} \mathbf{x} \\
& \left.+\int_{\Xi_{q}}[\mathbf{x}+(\mathbf{u} \cdot \mathbf{x}) \mathbf{u}](\mathbf{u}-\boldsymbol{\Omega} \cdot \mathbf{x}) \cdot \boldsymbol{A}(\boldsymbol{q}+\mathbf{x}) f_{\mathrm{e}}(\|\mathbf{x}\|) \delta^{\prime}(\mathbf{u} \cdot \mathbf{x}) \mathrm{d}^{4} \mathbf{x}\right\} \delta \boldsymbol{q} \mathrm{d} \tau \\
& -\int_{\tau_{1}}^{\tau_{2}} \int_{\Xi_{q}} \nabla_{\mathrm{g}} \otimes \boldsymbol{A}(\boldsymbol{q}+\mathbf{x}) \cdot[\boldsymbol{u}-\boldsymbol{\Omega} \cdot \mathbf{x}] f_{\mathrm{e}}(\|\mathbf{x}\|) \delta(\mathbf{u} \cdot \mathbf{x}) \mathrm{d}^{4} \mathbf{x} \delta \boldsymbol{q} \mathrm{d} \tau .
\end{aligned}
$$

We next simplify the previous equation with the help of the following useful identity. Performing an integration by parts, one can easily check that

$$
\begin{aligned}
& \int_{\Xi_{q}}[\boldsymbol{u}-\boldsymbol{\Omega} \cdot \boldsymbol{x}] \cdot \nabla_{\mathrm{g}} \otimes \boldsymbol{A}(\boldsymbol{q}+\mathbf{x}) f_{\mathrm{e}}(\|\mathbf{x}\|) \delta(\boldsymbol{u} \cdot \mathbf{x}) \mathrm{d}^{4} \boldsymbol{x} \\
& =\int_{\mathbb{R}^{1,3}} \boldsymbol{A}(\boldsymbol{q}+\mathbf{x})(\operatorname{Tr} \boldsymbol{\Omega}) f_{\mathrm{e}}(\|\mathbf{x}\|) \delta(\mathbf{u} \cdot \mathbf{x}) \mathrm{d}^{4} \mathbf{x} \\
& -\int_{\Xi_{q}} \boldsymbol{A}(\boldsymbol{q}+\mathbf{x})[\mathbf{u} \cdot \mathbf{x}-\mathbf{x} \cdot \boldsymbol{\Omega} \cdot \mathbf{x}] f_{\mathrm{e}}^{\prime}(\|\mathbf{x}\|) /\|\boldsymbol{x}\| \delta(\boldsymbol{u} \cdot \boldsymbol{x}) \mathrm{d}^{4} \mathbf{x} \\
& -\int_{\Xi_{q}} \boldsymbol{A}(\boldsymbol{q}+\mathbf{x}) \mathbf{u} \cdot[\mathbf{u}-\boldsymbol{\Omega} \cdot \boldsymbol{x}] f_{\mathrm{e}}(\|\mathbf{x}\|) \delta^{\prime}(\mathbf{u} \cdot \mathbf{x}) \mathrm{d}^{4} \mathbf{x} .
\end{aligned}
$$

By the anti-symmetry of $\Omega$ we have $\operatorname{Tr} \Omega=0$, so that the first integral of the r.h.s. of (A.33) vanishes. Again by the anti-symmetry of $\boldsymbol{\Omega}$, we also have that $\boldsymbol{x} \cdot \boldsymbol{\Omega} \cdot \boldsymbol{x}=0$, and furthermore we have $\mathbf{u} \cdot \mathbf{x} \delta(\mathbf{u} \cdot \mathbf{x})=0$. Therefore, the second integral of the r.h.s. of (A.33) vanishes, too. As for the last integral of the r.h.s. of (A.33), we use that $\Omega=\Omega_{\mathrm{E}}+\boldsymbol{\Omega}_{\mathrm{FW}}$ with $\boldsymbol{\Omega}_{\mathrm{FW}}=\stackrel{\circ}{\mathrm{u}} \wedge \mathbf{u}$, and moreover that $\boldsymbol{u} \cdot \boldsymbol{\Omega}_{\mathrm{E}}=0$, to rewrite it into

$$
\begin{aligned}
& -\int_{\Xi_{q}} \boldsymbol{A}(\boldsymbol{q}+\mathbf{x}) \mathbf{u} \cdot[\mathbf{u}-\boldsymbol{\Omega} \cdot \boldsymbol{x}] f_{\mathrm{e}}(\|\boldsymbol{x}\|) \delta^{\prime}(\mathbf{u} \cdot \boldsymbol{x}) \mathrm{d}^{4} \mathbf{x} \\
& =\quad \int_{\Xi_{q}} \boldsymbol{A}(\boldsymbol{q}+\mathbf{x})[1+\stackrel{\circ}{\mathbf{u}} \cdot \mathbf{x}] f_{\mathrm{e}}(\|\mathbf{x}\|) \delta^{\prime}(\mathbf{u} \cdot \mathbf{x}) \mathrm{d}^{4} \mathbf{x} .
\end{aligned}
$$

Finally, the r.h.s. of (A.34) is integrated by parts once, and we arrive at the final identity

$$
\begin{array}{r}
\int_{\Xi_{q}}[\mathbf{u}-\boldsymbol{\Omega} \cdot \mathbf{x}] \cdot \boldsymbol{\nabla}_{\mathrm{g}} \otimes \boldsymbol{A}(\boldsymbol{q}+\mathbf{x}) f_{\mathrm{e}}(\|\mathbf{x}\|) \delta(\boldsymbol{u} \cdot \mathbf{x}) \mathrm{d}^{4} \mathbf{x} \\
=\frac{\mathrm{d}}{\mathrm{d} \tau} \int_{\Xi_{q}} \boldsymbol{A}(\boldsymbol{q}+\mathbf{x}) f_{\mathrm{e}}(\|\mathbf{x}\|) \delta(\boldsymbol{u} \cdot \mathbf{x}) \mathrm{d}^{4} \mathbf{x} .
\end{array}
$$


This identity is now used to anti-symmetrize the last integral in (A.32). Noticing next that $\boldsymbol{\nabla}_{\mathrm{g}} \otimes \boldsymbol{A} \cdot \mathbf{v}-\mathbf{v} \cdot \boldsymbol{\nabla}_{\mathrm{g}} \otimes \boldsymbol{A}=\boldsymbol{\nabla}_{\mathrm{g}} \wedge \boldsymbol{A} \cdot \mathbf{v}$ for an arbitrary four-vector $\mathbf{v}$, and recalling that $\boldsymbol{\nabla}_{\mathrm{g}} \wedge \boldsymbol{A}=\mathbf{F}$, the variation (A.32) of $\mathcal{A}_{\text {coupl }}$ takes the form

$$
\delta \mathcal{A}_{\text {coupl }}=\int_{\tau_{1}}^{\tau_{2}}\left[{\stackrel{\circ}{\boldsymbol{p}_{\mathrm{N}}}}^{-\boldsymbol{f}}\right] \delta \boldsymbol{q} \mathrm{d} \tau
$$

where

$$
\boldsymbol{f}=\int_{\Xi_{q}} \mathbf{F}(\boldsymbol{q}+\mathbf{x}) \cdot(\boldsymbol{u}-\boldsymbol{\Omega} \cdot \mathbf{x}) f_{\mathrm{e}}(\|\mathbf{x}\|) \delta(\boldsymbol{u} \cdot \mathbf{x}) \mathrm{d}^{4} \boldsymbol{x}
$$

is the Abraham-Lorentz type Minkowski force, and

$$
\boldsymbol{p}_{\mathrm{N}}=-\int_{\Xi_{q}} \mathbf{x}\left[\mathbf{x} \cdot \boldsymbol{\Omega}_{\mathrm{E}} \cdot \mathbf{F}(\boldsymbol{q}+\mathbf{x}) \cdot \boldsymbol{u}\right] f_{\mathrm{e}}(\|\mathbf{x}\|) \delta(\boldsymbol{u} \cdot \mathbf{x}) \mathrm{d}^{4} \mathbf{x}
$$

is Nodvik's Minkowski momentum for the electromagnetic spin-orbit coupling. For later convenience, we notice that the charged particle's co-moving electromagnetic field, viz. its Coulomb field and its instantaneous gyro-magnetic field (both defined w.r.t. the FermiWalker frame) do not contribute to $\boldsymbol{p}_{\mathrm{N}}$, so that $\mathbf{F}$ in (A.38) can be replaced by the reduced field tensor $\mathbf{F}^{\prime}$, which obtains from $\mathbf{F}$ by subtracting the particle's co-moving electromagnetic field. Furthermore, making use of the identity $\boldsymbol{u} \cdot \boldsymbol{\Omega}_{\mathrm{E}}=\boldsymbol{O}$, and using the fact that terms proportional to $\mathbf{x} \cdot \boldsymbol{u}$ in the integrand vanish upon integration against $\delta(\boldsymbol{u} \cdot \mathbf{x})$, we can rewrite the four-vector $\boldsymbol{p}_{\mathrm{N}}$ in the more appealing format

$$
p_{\mathrm{N}}=\mathbf{M}_{\mathrm{N}} \cdot \mathbf{u}
$$

where we introduced the symmetric Nodvik tensor mass of spin-orbit coupling,

$$
\mathbf{M}_{\mathrm{N}}=-\int_{\Xi_{q}}\left[\mathbf{x} \otimes \mathbf{x},\left[\mathbf{F}^{\prime}(\boldsymbol{q}+\mathbf{x}), \boldsymbol{\Omega}_{\mathrm{E}}\right]_{+}\right]_{+} f_{\mathrm{e}}(\|\mathbf{x}\|) \delta(\boldsymbol{u} \cdot \mathbf{x}) \mathrm{d}^{4} \mathbf{x} .
$$

The Euler-Lagrange equation for $\delta\left(\mathcal{A}_{\text {bare }}+\mathcal{A}_{\text {coupl }}\right)=0$ under the variation of $\boldsymbol{q}(\tau)$ for given $\mathbf{F}(\mathbf{x})$ and $\boldsymbol{\Omega}_{\mathrm{E}}(\tau)$ can now be read off from (A.36) and (A.20) as

$$
\frac{\mathrm{d} \boldsymbol{p}}{\mathrm{d} \tau}=\boldsymbol{f}
$$

where

$$
\mathbf{p}=\mathbf{M} \cdot \mathbf{u},
$$

is the four-vector of the Minkowski momentum, with $\mathbf{M}=\mathbf{M}_{\mathrm{b}}+\mathbf{M}_{\mathrm{N}}$. Equation (A.41) is the Minkowski equation of motion with the expected Abraham-Lorentz type Minkowski force, but with a somewhat unusual anisotropic tensor mass $\mathbf{M}$. 


\section{A.1.4 Euler-Lagrange equations for the gyrograph}

Using (A.8) and integrating by parts, observing that the variations of the Euler variables vanish at the boundaries, for the first variation of the action $\mathcal{A}_{\text {bare }}$ of the bare particle we find

$$
\delta \mathcal{A}_{\text {bare }}=\frac{1}{2} \int_{\tau_{1}}^{\tau_{2}} \operatorname{Tr}\left(\left(\mathbf{S}_{\mathrm{b}}-\left[\mathbf{S}_{\mathrm{b}}, \boldsymbol{\Omega}\right]_{-}\right) \cdot \delta \boldsymbol{\theta}\right) \mathrm{d} \tau,
$$

and for the first variation of the coupling action we get

$$
\delta \mathcal{A}_{\text {coupl }}=\frac{1}{2} \int_{\tau_{1}}^{\tau_{2}} \operatorname{Tr}\left(\left(\mathbf{S}_{\mathrm{f}}-\left[\mathbf{S}_{\mathrm{f}}, \boldsymbol{\Omega}\right]_{-}\right) \cdot \delta \boldsymbol{\theta}\right) \mathrm{d} \tau
$$

where we introduced the tensor of electromagnetic Minkowski spin (about $\mathbf{q}$ ) of the charged particle,

$$
\mathbf{S}_{\mathrm{f}} \stackrel{\text { def }}{=} \int_{\Xi_{q}} \mathbf{x} \wedge \boldsymbol{A}(\boldsymbol{q}+\mathbf{x}) f_{\mathrm{e}}(\|\boldsymbol{x}\|) \delta(\boldsymbol{u} \cdot \mathbf{x}) \mathrm{d}^{4} \mathbf{x} .
$$

We remark that $\mathbf{S}_{\mathrm{f}}$ is invariant under a gauge transformation $\boldsymbol{A} \rightarrow \boldsymbol{A}+\boldsymbol{\nabla}_{\mathrm{g}} \Psi$. Defining now the total spin of the particle as

$$
\mathbf{S} \stackrel{\text { def }}{=} \mathbf{S}_{\mathrm{f}}+\mathbf{S}_{\mathrm{b}}
$$

and introducing the abbreviation

$$
\mathbf{N} \stackrel{\text { def }}{=} \frac{\mathrm{d}}{\mathrm{d} \tau} \mathbf{S}+[\Omega, \mathbf{S}]_{-},
$$

the vanishing of the first variation of the action functional with respect to all anti-symmetric $\delta \boldsymbol{\theta}$ that are of space-space type with respect to $\boldsymbol{u}$ now simply reads

$$
0=\int_{\tau_{1}}^{\tau_{2}} \operatorname{Tr}(\mathbf{N} \cdot \delta \boldsymbol{\theta}) \mathrm{d} \tau .
$$

At this point we recall that, given any tetrad, any anti-symmetric tensor $\mathbf{A}$ can be uniquely decomposed into a sum of its space-space and time-space parts. With $\boldsymbol{u}$ as the timelike unit vector of the tetrad, we have

$$
\mathbf{A}=\mathbf{A}^{\perp}+\mathbf{A}^{\|},
$$

where

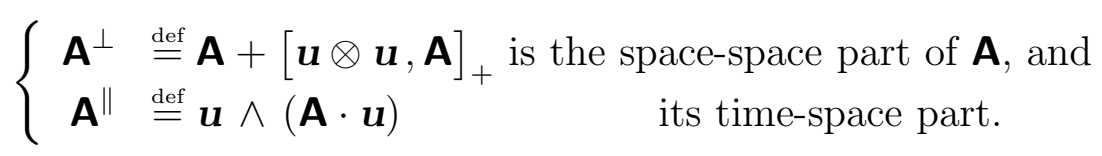

Armed with the above decomposition, we conclude that equation (A.47) can only be satisfied if the space-space part w.r.t. $\mathbf{u}$ of the anti-symmetric tensor $\mathbf{N}$ vanishes, explicitly

$$
\mathbf{N}^{\perp}=\mathbf{0},
$$


which is the Euler-Lagrange equation for the rotational variations.

To extract the evolution equation for $\Omega_{\mathrm{E}}$ from (A.50), we also decompose the antisymmetric spin tensor $\mathbf{S}$ into its space-space and time-space parts with respect to $\boldsymbol{u}$,

$$
\mathbf{S}=\mathbf{S}^{\perp}+\mathbf{S}^{\|} .
$$

Inserting the above decomposition of $\mathbf{S}$ into the definition (

$$
\mathbf{N}=\frac{\mathrm{d}}{\mathrm{d} \tau} \mathbf{S}^{\perp}+\left[\Omega, \mathbf{S}^{\perp}\right]_{-}+\frac{\mathrm{d}}{\mathrm{d} \tau} \mathbf{S}^{\|}+\left[\Omega, \mathbf{S}^{\|}\right]_{-} .
$$

With the help of the helicity four-vector $\boldsymbol{H} \stackrel{\text { def }}{=} \mathbf{S} \cdot \boldsymbol{u}$ we can write

$$
\left\{\begin{array}{l}
\mathbf{S}^{\perp}=\mathbf{S}-\mathbf{u} \wedge \boldsymbol{H} \\
\mathbf{S}^{\|}=\mathbf{u} \wedge \boldsymbol{H}
\end{array} .\right.
$$

By straightforward calculation we furthermore find that

$$
\frac{\mathrm{d}}{\mathrm{d} \tau} \mathbf{S}^{\|}+\left[\boldsymbol{\Omega}, \mathbf{S}^{\|}\right]_{-}=\mathbf{u} \wedge\left(\stackrel{\circ}{\boldsymbol{H}}+\boldsymbol{\Omega}_{\mathrm{E}} \cdot \boldsymbol{H}\right)
$$

and that

$$
\left(\frac{\mathrm{d}}{\mathrm{d} \tau} \mathbf{S}^{\perp}+\left[\Omega, \mathbf{S}^{\perp}\right]_{-}\right) \cdot \boldsymbol{u}=\boldsymbol{O} .
$$

Recalling next that $\mathbf{S}_{\mathrm{b}} \cdot \boldsymbol{u}=\boldsymbol{O}$ (i.e. $\mathbf{S}_{\mathrm{b}}{ }^{\|}=\boldsymbol{O}$, so that $\mathbf{S}_{\mathrm{b}}=\mathbf{S}_{\mathrm{b}}{ }^{\perp}$ ) we find that $\mathbf{S} \cdot \mathbf{u}=\mathbf{S}_{\mathrm{f}} \cdot \mathbf{u}$, viz.

$$
\boldsymbol{H}=\int_{\Xi_{q}} \mathbf{x} \boldsymbol{u} \cdot \boldsymbol{A}(\boldsymbol{q}+\mathbf{x}) f_{\mathrm{e}}(\|\mathbf{x}\|) \delta(\boldsymbol{u} \cdot \mathbf{x}) \mathrm{d}^{4} \mathbf{x},
$$

from where we read off that $\boldsymbol{H} \cdot \boldsymbol{u}=\boldsymbol{O}$, i.e. $\boldsymbol{H}$ is itself spacelike for all $\tau$. This implies that $\stackrel{\circ}{\boldsymbol{H}}+\boldsymbol{\Omega}_{\mathrm{E}} \cdot \boldsymbol{H}$ is spacelike, and we conclude that

$$
\begin{gathered}
\frac{\mathrm{d}}{\mathrm{d} \tau} \mathbf{S}^{\|}+\left[\Omega, \mathbf{S}^{\|}\right]_{-}=\mathbf{N}^{\|}, \\
\frac{\mathrm{d}}{\mathrm{d} \tau} \mathbf{S}^{\perp}+\left[\Omega, \mathbf{S}^{\perp}\right]_{-}=\mathbf{N}^{\perp} .
\end{gathered}
$$

Hence, the Euler-Lagrange equation (A.50) reads

$$
\frac{\mathrm{d}}{\mathrm{d} \tau} \mathbf{S}^{\perp}+\left[\Omega, \mathbf{S}^{\perp}\right]_{-}=\mathbf{0} .
$$

Remark: The conservation law for $\left\|\mathbf{S}^{\perp}\right\|$ follows immediately from (A.59).

It remains to show that (A.59) is identical to (7.6). We recall that $\mathbf{S}=\mathbf{S}_{\mathrm{b}}+\mathbf{S}_{\mathrm{f}}$, and notice that decomposition into space-space and time-space parts commutes with addition, so that $\mathbf{S}^{\perp}=\mathbf{S}_{\mathrm{b}}{ }^{\perp}+\mathbf{S}_{\mathrm{f}}{ }^{\perp}$. We also recall that $\mathbf{S}_{\mathrm{b}}{ }^{\perp}=\mathbf{S}_{\mathrm{b}}$. We furthermore notice that $\Omega_{\mathrm{E}} \propto \mathbf{S}_{\mathrm{b}}$, 
which implies that $\left[\boldsymbol{\Omega}_{\mathrm{E}}, \mathbf{S}_{\mathrm{b}}\right]_{-}=\boldsymbol{O}$, so that $\left[\boldsymbol{\Omega}, \mathbf{S}_{\mathrm{b}}\right]_{-}=\left[\boldsymbol{\Omega}_{\mathrm{FW}}, \mathbf{S}_{\mathrm{b}}\right]_{-}$. Using all these pieces of information we see that (A.59) states that

$$
\frac{\mathrm{d}}{\mathrm{d} \tau} \mathbf{S}_{\mathrm{b}}+\left[\boldsymbol{\Omega}_{\mathrm{FW}}, \mathbf{S}_{\mathrm{b}}\right]_{-}=-\frac{\mathrm{d}}{\mathrm{d} \tau} \mathbf{S}_{\mathrm{f}}^{\perp}-\left[\boldsymbol{\Omega}, \mathbf{S}_{\mathrm{f}}^{\perp}\right]_{-} .
$$

The 1.h.s. of (A.60) is manifestly identical to the 1.h.s. of (7.6). To see that the r.h.s. of (A.60) is identical to the r.h.s. of $(\sqrt{7.6})$, one can rewrite the calculation in Appendix C of [15] into our notation, using the Nodvik Maxwell-Lorentz equations (5.1), (5.2), (5.3), and a few integrations by parts. The details are not repeated here.

\section{A.2 Point mass and point charge limits of massive LED}

In the renormalization flow study of our massive LED with the choice (10.1) for the mass and charge densities, the renormalized particle reaches a finite smallest size $=1.5 R_{\mathrm{C}}$ in the 'renormalized purely electromagnetic' limit of vanishing bare rest mass $m_{\mathrm{b}} \rightarrow 0^{+}$. This renormalization program for massive LED aside, it is of interest to investigate which types of theories emerge in the point limits for mass and charge, either taken separately or jointly.

\section{A.2.1 The point mass limit}

In the point mass limit

$$
f_{\mathrm{m}}(\|\mathbf{x}\|) \longrightarrow m_{\mathrm{b}} \frac{1}{4 \pi\|\mathbf{x}\|^{2}} \delta(\|\mathbf{x}\|) \stackrel{\text { def }}{=} f_{\mathrm{m}}^{\text {NoDVIK }}(\|\mathbf{x}\|),
$$

but with $f_{\mathrm{e}}$ fixed and 'regular' (not a point charge density), and with the condition that $\left\|\boldsymbol{w}_{\mathrm{E}}\right\|<C$, our massive LED formally reduces to the massive LED of Nodvik [15]. In this limit, the square root terms in (4.2) and (6.5) become just factors of unity on the support of $f_{\mathrm{m}}^{\text {Nodvik }}$, so that the bare particle's gyrational mass (4.2) degenerates identically into a constant,

$$
\mathcal{M}_{\mathrm{b}}\left(\left\|\boldsymbol{w}_{\mathrm{E}}\right\|\right) \rightarrow m_{\mathrm{b}}
$$

and our bare action reduces simply to the action of a free mechanical point particle with bare rest mass $m_{\mathrm{b}}$,

$$
\int_{\Xi} \mathcal{L}_{\text {bare }}(\mathbf{x}) \mathrm{d}^{4} \mathbf{x} \longrightarrow-m_{\mathrm{b}} \int_{\tau_{1}}^{\tau_{2}} \mathrm{~d} \tau=\mathcal{A}_{\text {bare }}^{\text {NoDvIK }}
$$

employed by Nodvik [15]. The field and coupling actions remain the same, however. Accordingly, the electromagnetic part of the two models is identical, i.e. the Maxwell-Lorentz equations in Nodvik's massive LED are given by (5.1), (5.2), and (5.3). Moreover, the world-line equation in Nodvik's massive LED can still be written as

$$
\frac{\mathrm{d}}{\mathrm{d} \tau} \boldsymbol{p}(\tau)=\int_{\mathbb{R}^{1,3}} \mathbf{F}(\mathbf{x}) \cdot \boldsymbol{U}(\mathbf{x} ; \tau) f_{\mathrm{e}}(\|\mathbf{x}-\boldsymbol{q}(\tau)\|) \delta(\mathbf{u} \cdot(\boldsymbol{x}-\boldsymbol{q}(\tau))) \mathrm{d}^{4} \mathbf{x}
$$


but now one has

$$
\boldsymbol{p}(\tau)=\mathbf{M}^{\operatorname{Nodvik}}(\tau) \cdot \mathbf{u}(\tau)
$$

with

$$
\mathbf{M}^{\text {Nodvik }}(\tau)=m_{\mathrm{b}} \mathbf{g}-\int_{\mathbb{R}^{1,3}}\left[\boldsymbol{x} \otimes \mathbf{x},\left[\mathbf{F}(\boldsymbol{q}+\mathbf{x}), \boldsymbol{\Omega}_{\mathrm{E}}\right]_{+}\right]_{+} f_{\mathrm{e}}(\|\boldsymbol{x}\|) \delta(\mathbf{u} \cdot \mathbf{x}) \mathrm{d}^{4} \mathbf{x},
$$

which is a slightly simpler mass tensor than $\mathbf{M}$. On the other hand, the point mass limit eliminates the moment of inertia of the bare particle while the norm of $\boldsymbol{\Omega}_{\mathrm{E}}$ remains bounded (by hypothesis) so that $\mathbf{S}_{\mathrm{b}} \rightarrow \mathbf{0}$ identically; whence, the gyrograph equation (7.6) degenerates into the statement $\mathbf{t}=\mathbf{0}$, i.e., upon using (7.7),

$$
\mathbf{0}=\int_{\mathbb{R}^{1,3}}(\mathbf{x}-\boldsymbol{q}) \wedge(\mathbf{F}(\mathbf{x}) \cdot \boldsymbol{U}(\mathbf{x}, \tau))^{\perp} f_{\mathrm{e}}(\|\mathbf{x}-\boldsymbol{q}\|) \delta(\mathbf{u} \cdot(\mathbf{x}-\boldsymbol{q})) \mathrm{d}^{4} \mathbf{x} .
$$

These are the equations of the massive LED of Nodvik [15]. We will now explain that this is a qualitatively quite different dynamical theory from our massive LED.

First of all, since one loses a proper-time derivative for $\Omega_{\mathrm{E}}$ by passing from (7.6) to (A.67), the point mass limit is a singular limit from the dynamical perspective of the Cauchy problem for $\Omega_{\mathrm{E}}$. This fact in itself already means that it will no longer be possible to arbitrarily choose initial data for $\Omega_{\mathrm{E}}$ from the set of admissible Cauchy data for (7.6). Indeed, choose (which we always may) a Lorentz frame in which initially (i.e. at $t=0=\tau$ ) we have $\boldsymbol{q}_{0}=\boldsymbol{O}$ and $\mathbf{u}_{0}=\boldsymbol{e}_{0}$, and pick the fields $\mathbf{F}_{0}$ on $\boldsymbol{u}_{0} \cdot \boldsymbol{x}=0$ at random from our set of admissible Cauchy data for $\mathbf{F}_{0}$. Then (A.67) at $\tau=0$ has only a nontrivial space part, which in three-space notation reads

$$
\boldsymbol{t}_{0}^{E}+\boldsymbol{\omega}_{\mathrm{E} 0} \times \boldsymbol{\sigma}_{0}^{B}=\mathbf{0}
$$

where $\boldsymbol{\omega}_{\mathrm{E} 0}$ is the space part of the dual four-vector for $\boldsymbol{\Omega}_{\mathrm{E} 0}$, and where

$$
\boldsymbol{t}_{0}^{E \stackrel{\text { def }}{=}} \int_{\mathbb{R}^{3}} \boldsymbol{x} \times \mathcal{E}_{0}(\boldsymbol{x}) f_{\mathrm{e}}(|\boldsymbol{x}|) \mathrm{d}^{3} x
$$

is the initial electric torque on the particle and

$$
\boldsymbol{\sigma}_{0}^{B} \stackrel{\text { def }}{=} \int_{\mathbb{R}^{3}} \boldsymbol{x} \boldsymbol{x} \cdot \boldsymbol{\mathcal { B }}_{0}(\boldsymbol{x}) f_{\mathrm{e}}(|\boldsymbol{x}|) \mathrm{d}^{3} x
$$

its initial 'magnetic spin.' Clearly, any attempt to also prescribe Cauchy data for the angular velocity tensor $\Omega_{\mathrm{E} 0}$, chosen at random from our set of admissible data, will almost surely violate (A.68).

More specifically, (A.68), with (A.69) and (A.70), imposes not just on the set of admissible initial data for $\boldsymbol{\Omega}_{\mathrm{E}}$ but also on the fields $\mathbf{F}_{0}$, in the sense that for most choices of $\mathbf{F}_{0}$ no choice for $\Omega_{\mathrm{E} 0}$ will satisfy (A.68). Thus, one extracts directly from (A.68), (A.69), (A.70) that the originally admissible field data are now further restricted as follows: either $\boldsymbol{\mathcal { B }}_{0}$ satisfies $\boldsymbol{\sigma}_{0}^{B}=\mathbf{0}$, and in this case also $\boldsymbol{E}_{0}$ has to satisfy $\boldsymbol{t}_{0}^{E}=\mathbf{0}$; or $\boldsymbol{\mathcal { B }}_{0}$ satisfies $\boldsymbol{\sigma}_{0}^{B} \neq \mathbf{0}$, in which 
case $\mathcal{E}_{0}$ has to satisfy $\boldsymbol{t}_{0}^{E} \cdot \boldsymbol{\sigma}_{0}^{B}=0$. Only if these alternate constraints on the initial fields are satisfied is it possible to satisfy (A.68) by a compatible class of initial data $\boldsymbol{\Omega}_{\mathrm{E} 0}$ (viz., $\boldsymbol{\omega}_{\mathrm{E} 0}$ ). Namely, if $\boldsymbol{\sigma}_{0}^{B}=\mathbf{0}$ and $\boldsymbol{t}_{0}^{E}=0$, then the admissible data for $\boldsymbol{\omega}_{\mathrm{E} 0}$ are the same as for our model. If $\boldsymbol{\sigma}_{0}^{B} \neq \mathbf{0}$ and $\boldsymbol{t}_{0}^{E} \cdot \boldsymbol{\sigma}_{0}^{B}=0$, however, then $\boldsymbol{\omega}_{\mathrm{E} 0}$ has to be of the form

$$
\boldsymbol{\omega}_{\mathrm{E} 0}=\frac{1}{\left|\boldsymbol{\sigma}_{0}^{B}\right|^{2}} \boldsymbol{t}_{0}^{E} \times \boldsymbol{\sigma}_{0}^{B}+\alpha \boldsymbol{\sigma}_{0}^{B}
$$

which leaves only one degree of freedom to choose from instead of three, i.e. the coefficient $\alpha$ in front of $\boldsymbol{\sigma}_{0}^{B}$ is at our disposal. This clarifies to which extent initial data for Nodvik's model have to be compatible.

Compatibility of a set of initial data in the sense just explained does not, however, imply that all is well then, for not only has one lost a proper-time derivative on $\boldsymbol{\Omega}_{\mathrm{E}}$ by passing from (7.6) to (A.67), a proper-time derivative of $\Omega_{\mathrm{E}}$ does not show any more at all in (A.67). Nodvik's (A.67) is therefore not an orderly evolution equation for $\Omega_{\mathrm{E}}$, but rather a constraint equation for the dynamical field variables $\mathbf{F}$ and the dynamical particle variables $\boldsymbol{q}$ and $\mathbf{u}$, which in case of actually being satisfied by these variables for some time interval after the initial instant, still leaves a certain amount of freedom for choosing the value of the now purely kinematical variable $\Omega_{\mathrm{E}}$. Therefore, to the extent that compatible initial data for $\mathbf{F}, \boldsymbol{q}$, and $\boldsymbol{u}$, with a compatible initial choice of $\boldsymbol{\Omega}_{\mathrm{E}}$, can be continued at all according to Nodvik's equations, such a continuation is presumably not unique.

Remark: We shall see that a similar assessment holds for the semi-relativistic, purely electromagnetic theory of Abraham, which is discussed in Appendix A.3.

\section{A.2.2 Remarks on the spinless point particle limit}

Ever since Dirac's surmise [61] that, as long as we are willing to let the bare mass take the value $-\infty$ to compensate for the $+\infty$ electromagnetic self-energies, we may view the Abraham-Lorentz-Dirac (ALD) equation

$$
m \stackrel{\circ}{\mathbf{q}}=-e \mathbf{F}^{\mathrm{ext}} \cdot \stackrel{\circ}{\mathbf{q}}+\frac{2}{3} \frac{e^{2}}{c^{3}}(\mathbf{g}+\stackrel{\circ}{\mathbf{q}} \otimes \stackrel{\circ}{\mathbf{q}}) \cdot \stackrel{\circ}{\mathbf{q}}
$$

as the exact equation for the radiation reaction-corrected motion of a spinless point particle with empirical electron mass $m$ and empirical electron charge $-e$ in external electromagnetic fields $\mathbf{F}^{\text {ext }}$, there has been a strong interest in making sense out of the peculiar third-order proper-time derivative of $\boldsymbol{q}$ that figures in (A.72), e.g. [27, 61, 62, 63, 64, 65]. A rigorous clarification of Dirac's surmise is, however, not so easy to come by. Notable rigorous attempts to construct a consistent renormalized electrodynamics with spinless point charges are [19 and [66]; see also 67] and 68, 69] for work on the classical Pauli-Fierz model, a semirelativistic dipole approximation to the problem, which also serves as point of departure for setting up a so-called non-relativistic QED [53].

Naturally one may wonder whether (A.72) emerges in some spinless point particle limit of our massive LED, or to begin simpler, in the point particle limit of a spinless version of our

massive LED. This massive LED without spin obtains from our model by simply discarding 
(7.6) and setting $\boldsymbol{\Omega}_{\mathrm{E}} \equiv \mathbf{0}$ in the remaining equations. Thus, the relativistic Maxwell-Lorentz equations are once again

$$
\nabla_{\mathrm{g}} \cdot{ }^{\star} \mathbf{F}=\mathbf{O}
$$

for the Hodge dual of $\mathbf{F}$, and

$$
\boldsymbol{\nabla}_{\mathrm{g}} \cdot \mathbf{F}(\mathbf{x})=4 \pi \int_{-\infty}^{+\infty} \boldsymbol{U}(\mathbf{x} ; \tau) f_{\mathrm{e}}(\|\mathbf{x}-\boldsymbol{q}(\tau)\|) \delta(\boldsymbol{u}(\tau) \cdot(\mathbf{x}-\boldsymbol{q}(\tau))) \mathrm{d} \tau
$$

for $\mathbf{F}$ itself, but now with

$$
\boldsymbol{U}(\mathbf{x} ; \tau)=\mathbf{u}(\tau)-(\stackrel{\circ}{\mathbf{u}}(\tau) \wedge \mathbf{u}(\tau)) \cdot(\mathbf{x}-\mathbf{q}(\tau))
$$

The world-line equation still reads

$$
\frac{\mathrm{d}}{\mathrm{d} \tau} \boldsymbol{p}=\int_{\mathbb{R}^{1,3}} \mathbf{F}(\mathbf{x}) \cdot \boldsymbol{U}(\mathbf{x} ; \tau) f_{\mathrm{e}}(\|\mathbf{x}-\boldsymbol{q}(\tau)\|) \delta(\boldsymbol{u}(\tau) \cdot(\mathbf{x}-\boldsymbol{q}(\tau))) \mathrm{d}^{4} \boldsymbol{x}
$$

but now with

$$
\boldsymbol{p}(\tau)=m_{\mathrm{b}} \mathbf{u}(\tau)
$$

and with $\boldsymbol{U}$ given in (A.75). Notice that the structure of $f_{\mathrm{m}}$ does not figure in this spinless LED, which is therefore identical to the spinless version of Nodvik's LED, due to Spohn [43], in which $f_{\mathrm{m}}$ is a point mass distribution from the outset. The question then is: What happens if we let $f_{\mathrm{e}}$ tend to a delta function concentrated at the same point? The first step to the answer is the rigorous control of this spinless massive LED which, however, is not very developed yet.

\section{A.3 Semi-relativistic LED}

In this Appendix we give a brief summary of semi-relativistic LED. This material can also be used as a primer for the relativistic theory. We begin with the massive model with spin. Its limiting cases of the more familiar Abraham model and the popular 'model without spin' are discussed in subsequent subsections.

\section{A.3.1 Massive model with spin}

As in the relativistic theory, the electric field $\mathcal{E}(\boldsymbol{x}, t) \in \mathbb{R}^{3}$ and the magnetic field $\mathcal{B}(\boldsymbol{x}, t) \in \mathbb{R}^{3}$ at the point $\boldsymbol{x} \in \mathbb{R}^{3}$ at time $t \in \mathbb{R}$ satisfy the classical Maxwell-Lorentz equations,

$$
\begin{aligned}
\boldsymbol{\nabla} \cdot \boldsymbol{B}(\boldsymbol{x}, t) & =0 \\
\frac{1}{c} \frac{\partial}{\partial t} \mathcal{B}(\boldsymbol{x}, t)+\nabla \times \mathcal{E}(\boldsymbol{x}, t) & =\mathbf{0} \\
\boldsymbol{\nabla} \cdot \mathcal{E}(\boldsymbol{x}, t) & =4 \pi \rho(\boldsymbol{x}, t), \\
-\frac{1}{c} \frac{\partial}{\partial t} \mathcal{E}(\boldsymbol{x}, t)+\boldsymbol{\nabla} \times \boldsymbol{B}(\boldsymbol{x}, t) & =4 \pi \rho(\boldsymbol{x}, t) \frac{1}{c} \boldsymbol{v}(\boldsymbol{x}, t),
\end{aligned}
$$


Although not manifestly Lorentz co-variant, the Maxwell-Lorentz equations (A.78), (A.79), (A.80), (A.81) appear to have a relativistic format already, but whether in fact they do satisfy relativistic Lorentz transformation laws or not depends on the model for the charge distribution. In the classical works of Abraham [1, 2] before the dawn of special relativity [3, 因, 6, 8], the electrical charge and current densities formally combine as $\rho(\boldsymbol{x}, t)(c, \boldsymbol{v}(\boldsymbol{x}, t))$ but do not transform like a relativistic four-current density vector.

In the semi-relativistic formulation of Abraham, the charge of a particle ( $-e$ for a model of the electron) is distributed around the instantaneous location $\boldsymbol{q}(t) \in \mathbb{R}^{3}$ of the particle by a charge density $f_{\mathrm{e}}$ with $S O(3)$ symmetry and compact support, satisfying $\int_{\mathbb{R}^{3}} f_{\mathrm{e}}(|\boldsymbol{x}|) \mathrm{d}^{3} x=-e$. This charge density is rigidly carried along by the particle with linear velocity $\dot{\boldsymbol{q}}(t)$ and rotating rigidly with angular velocity $\boldsymbol{\omega}(t)$. Thus, with a single charged particle source in (A.80), (A.81), we have

$$
\rho(\boldsymbol{x}, t)=f_{\mathrm{e}}(|\boldsymbol{x}-\boldsymbol{q}(t)|),
$$

and

$$
\boldsymbol{v}(\boldsymbol{x}, t)=\dot{\boldsymbol{q}}(t)+\boldsymbol{\omega}(t) \times(\boldsymbol{x}-\boldsymbol{q}(t)) .
$$

The evolution equations for the dynamical variables of the particle, i.e. position $\boldsymbol{q}(t)$, linear velocity $\dot{\boldsymbol{q}}(t)$, and angular velocity $\boldsymbol{\omega}(t)$, are likewise not Lorentz covariant and given by Newton's and Euler's evolution equations equipped with the Abraham-Lorentz expressions for the volume-averaged Lorentz force and torque [70], respectively, that act on the charged particle. Newton's equation of motion here reads

$$
\frac{\mathrm{d} \boldsymbol{p}_{\mathrm{b}}}{\mathrm{d} t}=\int_{\mathbb{R}^{3}}\left[\mathcal{E}(\boldsymbol{x}, t)+\frac{1}{c} \boldsymbol{v}(\boldsymbol{x}, t) \times \boldsymbol{B}(\boldsymbol{x}, t)\right] f_{\mathrm{e}}(|\boldsymbol{x}-\boldsymbol{q}(t)|) \mathrm{d}^{3} x,
$$

where

$$
\boldsymbol{p}_{\mathrm{b}}=m_{\mathrm{b}} \dot{\boldsymbol{q}}
$$

is the Newtonian linear particle momentum, with $m_{\mathrm{b}}$ the bare mass ('material mass' in [13]). Euler's equation of motion reads

$$
\frac{\mathrm{d} \boldsymbol{s}_{\mathrm{b}}}{\mathrm{d} t}=\int_{\mathbb{R}^{3}}(\boldsymbol{x}-\boldsymbol{q}(t)) \times\left[\boldsymbol{E}(\boldsymbol{x}, t)+\frac{1}{c} \boldsymbol{v}(\boldsymbol{x}, t) \times \boldsymbol{B}(\boldsymbol{x}, t)\right] f_{\mathrm{e}}(|\boldsymbol{x}-\boldsymbol{q}(t)|) \mathrm{d}^{3} x,
$$

where

$$
s_{\mathrm{b}}=I_{\mathrm{b}} \omega
$$

is the classical particle spin associated with the bare moment of inertia $I_{\mathrm{b}}$.

With the bare inertias $m_{\mathrm{b}} \neq 0$ and $I_{\mathrm{b}} \neq 0$, the semi-relativistic equations listed above pose a Cauchy problem for the following initial data at time $t=t_{0}$ : for the mechanical variables of the particles, the data are $\boldsymbol{q}\left(t_{0}\right), \dot{\boldsymbol{q}}\left(t_{0}\right)$ (equivalently: $\boldsymbol{p}_{\mathrm{b}}\left(t_{0}\right)$ ), and $\boldsymbol{\omega}\left(t_{0}\right)$ (equivalently: $\left.\boldsymbol{s}_{\mathrm{b}}\left(t_{0}\right)\right)$; for the fields, the data are $\mathcal{B}\left(\boldsymbol{x}, t_{0}\right)$ satisfying (A.78) at $t=t_{0}$, and $\mathcal{E}\left(\boldsymbol{x}, t_{0}\right)$ satisfying 
(A.80) at $t=t_{0}$. Notice that $(\mathrm{A.78})$ and (A.80) are merely initial constraints, which then remain satisfied by the fields $\mathcal{B}(\boldsymbol{x}, t)$ and $\mathcal{E}(\boldsymbol{x}, t)$ in the ensuing evolution. For (A.78) this is seen by taking the divergence of $(\mathrm{A.79})$. For $(\mathrm{A} .80$ ) this is seen by taking the divergence of (A.81) and the time-derivative of (A.80), then using the continuity equation for the charge,

$$
\partial_{t} \rho(\boldsymbol{x}, t)+\nabla \cdot(\rho(\boldsymbol{x}, t) \boldsymbol{v}(\boldsymbol{x}, t))=0,
$$

which is demanded by the Maxwell-Lorentz equations and indeed satisfied for $\rho(\boldsymbol{x}, t)$ given by $(\mathrm{A} .82$ ) and $\boldsymbol{v}(\boldsymbol{x}, t)$ by (A.83); cf. [71.

The semi-relativistic massive LED with spin has the following conservation laws [71. Charge,

$$
-e=\int_{\mathbb{R}^{3}} \rho(\boldsymbol{x}, t) \mathrm{d}^{3} x
$$

energy,

$$
W=\frac{1}{8 \pi} \int_{\mathbb{R}^{3}}\left(|\mathcal{E}|^{2}+|\mathcal{B}|^{2}\right) \mathrm{d}^{3} x+\frac{1}{2} \frac{\left|\boldsymbol{s}_{\mathrm{b}}\right|^{2}}{I_{\mathrm{b}}}+\frac{1}{2} \frac{\left|\boldsymbol{p}_{\mathrm{b}}\right|^{2}}{m_{\mathrm{b}}},
$$

linear momentum,

$$
\mathcal{P}=\frac{1}{4 \pi c} \int_{\mathbb{R}^{3}} \mathcal{E} \times \mathcal{B} \mathrm{d}^{3} x+\boldsymbol{p}_{\mathrm{b}}
$$

and angular momentum,

$$
\mathcal{L}=\frac{1}{4 \pi c} \int_{\mathbb{R}^{3}} \boldsymbol{x} \times(\mathcal{E} \times \mathcal{B}) \mathrm{d}^{3} x+\boldsymbol{q} \times \boldsymbol{p}_{\mathrm{b}}+\boldsymbol{s}_{\mathrm{b}}
$$

are all conserved during the evolution.

More detailed studies of the coupled dynamics of the massive semi-relativistic LED with spin have only recently begun.

\section{A.3.2 Massive model 'without spin'}

A mathematically regular limit which, unfortunately, is not quite physical but brings about some considerable simplification of the dynamical equations is to send $I_{\mathrm{b}} \rightarrow \infty$ while $m_{\mathrm{b}}$ is fixed at some finite value, in such a way that $\boldsymbol{s}_{\mathrm{b}}$ converges to some finite vector-valued function of $t$, which of course implies that $\boldsymbol{\omega} \rightarrow \mathbf{0}$. The semi-relativistic Maxwell-Lorentz equations are again given by (A.78), (A.80), (A.79), (A.81), and the charge density is still (A.82), but the current density vector now simplifies to

$$
\boldsymbol{j}(\boldsymbol{x}, t)=\rho(\boldsymbol{x}, t) \dot{\boldsymbol{q}}(t) .
$$

Moreover, the Newtonian equation of motion (A.102) reduces to

$$
\frac{\mathrm{d} \boldsymbol{p}_{\mathrm{b}}}{\mathrm{d} t}=\int_{\mathbb{R}^{3}}\left[\mathcal{E}(\boldsymbol{x}, t)+\frac{1}{c} \dot{\boldsymbol{q}}(t) \times \boldsymbol{B}(\boldsymbol{x}, t)\right] f_{\mathrm{e}}(|\boldsymbol{x}-\boldsymbol{q}(t)|) \mathrm{d}^{3} x,
$$


with $\boldsymbol{p}$ still given by (A.85), and the Eulerian equation (A.103) reduces to

$$
\frac{\mathrm{d} \boldsymbol{s}_{\mathrm{b}}}{\mathrm{d} t}=\int_{\mathbb{R}^{3}}(\boldsymbol{x}-\boldsymbol{q}(t)) \times\left[\boldsymbol{E}(\boldsymbol{x}, t)+\frac{1}{c} \dot{\boldsymbol{q}}(t) \times \mathcal{B}(\boldsymbol{x}, t)\right] f_{\mathrm{e}}(|\boldsymbol{x}-\boldsymbol{q}(t)|) \mathrm{d}^{3} x .
$$

In this limit, the Maxwell-Lorentz equations (A.78), (A.80), (A.79), (A.81), equipped with the charge density (A.82) and current density vector (A.93), and the Newtonian equation of motion (A.94), form a non-linearly coupled, closed system of equations, with initial data at $t=t_{0}$ given by: $\boldsymbol{q}\left(t_{0}\right)$ and $\dot{\boldsymbol{q}}\left(t_{0}\right)$ (equivalently: $\boldsymbol{p}_{\mathrm{b}}\left(t_{0}\right)$ ) for the particle; for the fields, the data are $\mathcal{B}\left(\boldsymbol{x}, t_{0}\right)$ satisfying (A.78) at $t=t_{0}$, and $\mathcal{E}\left(\boldsymbol{x}, t_{0}\right)$ satisfying (A.80) at $t=t_{0}$. Spin in turn adjusts passively to this combined field-particle dynamics according to a linear equation (A.95), which has to be supplemented by the initial data $\boldsymbol{s}_{\mathrm{b}}\left(t_{0}\right)$ (now of course no longer equivalent to prescribing $\boldsymbol{\omega}\left(t_{0}\right)$ ). Consequently, in this limit spin drops from the conservation laws - with the exception of the law for the angular momentum. Thus, the conserved energy $W$, linear momentum $\mathcal{P}$, and angular momentum $\mathcal{L}$ are now given by

$$
\begin{aligned}
& W=\frac{1}{8 \pi} \int_{\mathbb{R}^{3}}\left(|\mathcal{E}|^{2}+|\boldsymbol{B}|^{2}\right) \mathrm{d}^{3} x+\frac{1}{2} \frac{\left|\boldsymbol{p}_{\mathrm{b}}\right|^{2}}{m_{\mathrm{b}}}, \\
& \mathcal{P}=\frac{1}{4 \pi c} \int_{\mathbb{R}^{3}} \mathcal{E} \times \mathcal{B} \mathrm{d}^{3} x+\boldsymbol{p}_{\mathrm{b}},
\end{aligned}
$$

and

$$
\mathcal{L}=\frac{1}{4 \pi c} \int_{\mathbb{R}^{3}} \boldsymbol{x} \times(\mathcal{E} \times \mathcal{B}) \mathrm{d}^{3} x+\boldsymbol{q} \times \boldsymbol{p}_{\mathrm{b}}+\boldsymbol{s}_{\mathrm{b}}
$$

Notice that the laws for energy and linear momentum do not feature $\boldsymbol{s}_{\mathrm{b}}$, but the conservation law for the angular momentum does. Furthermore, the continuity equation simplifies to

$$
\partial_{t} \rho(\boldsymbol{x}, t)+\nabla \cdot(\rho(\boldsymbol{x}, t) \dot{\boldsymbol{q}}(t))=0,
$$

but this does not affect the law of charge conservation, which is still given by (A.89).

Since, in the limit $I_{\mathrm{b}} \rightarrow \infty$, spin is of no dynamical relevance, apart from the law of angular conservation, (A.95) is frequently omitted from the system of equations. The resulting subset of equations defines the semi-relativistic massive LED 'without spin.' This model without spin is still invariant under rotations, and by E. Noether's theorems there exists a conserved quantity associated with this invariance; only this quantity does not qualify as the physical angular momentum in the conventional sense. The observation that discarding the gyroscopic equation (A.95) violates the conventional law of angular momentum conservation (A.98) was made in [71].

The semi-relativistic massive LED without spin is the most thoroughly understood version of LED. In particular, we mention the recent rigorous treatments in [72, 73, 74, 75, 76], where for technical reasons the Einsteinian linear momentum

$$
\boldsymbol{p}_{\mathrm{b}}=\frac{m_{\mathrm{b}} \dot{\boldsymbol{q}}}{\sqrt{1-|\dot{\boldsymbol{q}}|^{2} / c^{2}}}
$$


with $m_{\mathrm{b}} \neq 0$ is used instead of the Newtonian (A.85) (see also 77, 78 for work on a simpler scalar field-particle theory). In this case, of course also the Newtonian kinetic energy in (A.90) is to be replaced by its Einsteinian counterpart

$$
\frac{1}{2} \frac{\left|\boldsymbol{p}_{\mathrm{b}}\right|^{2}}{m_{\mathrm{b}}} \longrightarrow m_{\mathrm{b}} c^{2} \sqrt{1+\frac{\left|\boldsymbol{p}_{\mathrm{b}}\right|^{2}}{m_{\mathrm{b}}^{2} c^{2}}}
$$

In [72, 73, the global existence and uniqueness for the Cauchy problem of a particle without spin and $m_{\mathrm{b}} \neq 0$ was proven. Moreover, in 72$]$ it was shown that the motion is stable if $m_{\mathrm{b}}>0$ and unstable if $m_{\mathrm{b}}<0$. The papers [73, 74, 75, 76] address the long time asymptotics of the slow motion of a spinless particle with $m_{\mathrm{b}}>0$ in slowly varying external fields. Using center manifold theory, these authors derive effective equations of motion of second order in the time derivative. Originally these effective second-order equations were obtained in Landau-Lifshitz [14] by applying a heuristic closure argument to the (in)famous third-order term in the Abraham-Lorentz-Dirac (ALD) equation (A.72), which works equally well for the relativistic and for the semi-relativistic version. As far as we know, the rigorous work of Spohn and collaborators gives the first clean derivation of the Landau-Lifshitz closure for the ALD equation, so far in its semi-relativistic setting.

\section{A.3.3 The Abraham model with spin}

Abraham's 11, 2] purely electromagnetic model obtains from the massive semi-relativistic LED with spin in the formal double limit $m_{\mathrm{b}} \rightarrow 0$ and $I_{\mathrm{b}} \rightarrow 0$ together with the assumption that the velocity $\dot{\boldsymbol{q}}$ and angular velocity $\boldsymbol{\omega}$ of the particle remain bounded. The MaxwellLorentz equations are not affected by this limit and still given by (A.78), (A.79), A.80), (A.81) together with (A.82) and (A.83). However, the bare Newtonian momentum $\boldsymbol{p}_{\mathrm{b}} \rightarrow \mathbf{0}$ and bare Eulerian spin $\boldsymbol{s}_{\mathrm{b}} \rightarrow \mathbf{0}$ identically in this limit. As a consequence, the evolution equations (A.84) and (A.86) degenerate into

$$
\mathbf{0}=\int_{\mathbb{R}^{3}}\left[\boldsymbol{E}(\boldsymbol{x}, t)+\frac{1}{c} \boldsymbol{v}(\boldsymbol{x}, t) \times \mathcal{B}(\boldsymbol{x}, t)\right] f_{\mathrm{e}}(|\boldsymbol{x}-\boldsymbol{q}(t)|) \mathrm{d}^{3} x
$$

and

$$
\mathbf{0}=\int_{\mathbb{R}^{3}}(\boldsymbol{x}-\boldsymbol{q}(t)) \times\left[\boldsymbol{\mathcal { E }}(\boldsymbol{x}, t)+\frac{1}{c} \boldsymbol{v}(\boldsymbol{x}, t) \times \boldsymbol{B}(\boldsymbol{x}, t)\right] f_{\mathrm{e}}(|\boldsymbol{x}-\boldsymbol{q}(t)|) \mathrm{d}^{3} x .
$$

The conservation laws for energy, linear and angular momentum simplify accordingly, because $\boldsymbol{p}_{\mathrm{b}}$ and $\boldsymbol{s}_{\mathrm{b}}$ vanish quadratically fast with $m_{\mathrm{b}} \rightarrow 0$ and $I_{\mathrm{b}} \rightarrow 0$ while $\dot{\boldsymbol{q}}$ and $\boldsymbol{\omega}$ remain bounded (by hypothesis!). Thus, the conserved energy $W$, linear momentum $\mathcal{P}$, and angular momentum $\mathcal{L}$ are now given by

$$
\begin{aligned}
& W=\frac{1}{8 \pi} \int_{\mathbb{R}^{3}}\left(|\mathcal{E}|^{2}+|\mathcal{B}|^{2}\right) \mathrm{d}^{3} x, \\
& \mathcal{P}=\frac{1}{4 \pi c} \int_{\mathbb{R}^{3}} \mathcal{E} \times \mathcal{B} \mathrm{d}^{3} x,
\end{aligned}
$$


and

$$
\mathcal{L}=\frac{1}{4 \pi c} \int_{\mathbb{R}^{3}} \boldsymbol{x} \times(\mathcal{E} \times \mathcal{B}) \mathrm{d}^{3} x
$$

The continuity equation (A.88) and its offspring, the law for the charge (A.89), still hold as stated.

This purely electromagnetic Abraham model with spin is not well-studied at all. In fact, it is hardly mentioned in textbooks and monographs on classical electromagnetism, with the notable exception of the book by Miller [5].

Subjecting equations (A.102) and (A.103) to closer scrutiny reveals that Abraham's purely electromagnetic semi-relativistic LED with spin is qualitatively a very different dynamical theory from the massive semi-relativistic LED with spin. In fact, since the coefficients in front of the time derivative of both $\dot{\boldsymbol{q}}$ and $\boldsymbol{\omega}$ vanish, these equations constitute a singular limit of the regular evolution equations (A.84) and (A.86), a limit in which Cauchy data for the dynamical particle variables $\dot{\boldsymbol{q}}$ and $\boldsymbol{\omega}$ can no longer be prescribed freely; also the field data are severely restricted. Only the initial particle position can be prescribed arbitrarily in Abraham's model. The situation is similar as in Nodvik's relativistic model, only worse.

More specifically, choose the initial time to be $t=0$ and the initial particle position to be $\boldsymbol{q}(0)=\mathbf{0}$, which can always be achieved by at most a time and a space translation. As before, denote the initial fields by $\boldsymbol{E}_{0}(\boldsymbol{x})$ and $\boldsymbol{B}_{0}(\boldsymbol{x})$. Then (A.102) demands that at $t=0$, we have

$$
c\left\langle\mathcal{E}_{0}\right\rangle+\dot{\boldsymbol{q}}_{0} \times\left\langle\mathcal{B}_{0}\right\rangle-\left(\left\langle\boldsymbol{x} \otimes \mathcal{B}_{0}\right\rangle-\left(\operatorname{tr}\left\langle\boldsymbol{x} \otimes \mathcal{B}_{0}\right\rangle\right) \mathbf{I}\right) \cdot \boldsymbol{\omega}_{0}=\mathbf{0},
$$

while (A.103) demands (as for Nodvik's model) that at $t=0$ we have

$$
c\left\langle\boldsymbol{x} \times \mathcal{E}_{0}\right\rangle+\boldsymbol{\omega}_{0} \times\left\langle\boldsymbol{x} \boldsymbol{x} \cdot \mathcal{B}_{0}\right\rangle=\mathbf{0} .
$$

Here, the averaging is defined by $\langle g\rangle \stackrel{\text { def }}{=}(-e)^{-1} \int_{\mathbb{R}^{3}} g(\boldsymbol{x}) f_{\mathrm{e}}(|\boldsymbol{x}|) \mathrm{d}^{3} x$, where we assume with Abraham that $(-e)^{-1} f_{\mathrm{e}}(|\boldsymbol{x}|) \geqslant 0$; furthermore, $\otimes$ now means the three-tensor product. One readily deduces from (A.107) and (A.108) that only the following subset of the originally admissible field data is now admitted. Namely, if $\left\langle\boldsymbol{x} \boldsymbol{x} \cdot \mathcal{B}_{0}\right\rangle=\mathbf{0}$, then also $\left\langle\boldsymbol{x} \times \mathcal{E}_{0}\right\rangle=\mathbf{0}$ must hold; if $\left\langle\boldsymbol{x} \boldsymbol{x} \cdot \mathcal{B}_{0}\right\rangle \neq \mathbf{0}$, then $\left\langle\boldsymbol{x} \times \mathcal{E}_{0}\right\rangle \cdot\left\langle\boldsymbol{x} \boldsymbol{x} \cdot \mathcal{B}_{0}\right\rangle=0$ must hold; if $\left\langle\boldsymbol{B}_{0}\right\rangle=\mathbf{0}$, then $\left\langle\mathcal{E}_{0}\right\rangle$ must be in the orthogonal complement of the left kernel space of $\left\langle\boldsymbol{x} \otimes \mathcal{B}_{0}\right\rangle-\left(\operatorname{tr}\left\langle\boldsymbol{x} \otimes \mathcal{B}_{0}\right\rangle\right) \mathbf{I}$, unless this tensor is the zero tensor, in which case $\left\langle\boldsymbol{E}_{0}\right\rangle$ must vanish also; if $\left\langle\boldsymbol{\mathcal { B }}_{0}\right\rangle \neq \mathbf{0}$ is in the left kernel space of $\left\langle\boldsymbol{x} \otimes \mathcal{B}_{0}\right\rangle-\left(\operatorname{tr}\left\langle\boldsymbol{x} \otimes \mathcal{B}_{0}\right\rangle\right) \mathbf{I}$, then also $\left\langle\mathcal{E}_{0}\right\rangle \cdot\left\langle\mathcal{B}_{0}\right\rangle=0$ must hold.

Remark: The above set of conditions on the initial fields imply in particular that the Abraham equations admit no solution for the following setup: $\boldsymbol{q}_{0}=\mathbf{0}, \boldsymbol{B}_{0}=\mathbf{0}$, and $\mathcal{E}_{0}=$ $\mathcal{E}^{\text {hom }}+\mathcal{E}^{\text {Couloms }}$, i.e. the linear superposition of the charge's radial symmetric Coulomb field with a homogeneous external field. In this case no choice of $\dot{\boldsymbol{q}}_{0}$ and $\boldsymbol{\omega}_{0}$ will rescue (A.107); in short, these perfectly physical initial conditions are inconsistent for the Abraham model.

We continue with the discussion of consistent initial data. Given now consistent data for the fields in accordance with the restrictions just explained, the initial data for $\dot{\boldsymbol{q}}_{0}$ and $\boldsymbol{\omega}_{0}$ 
are arbitrary only if $\left\langle\mathcal{B}_{0}\right\rangle=\mathbf{0}$ and $\left\langle\boldsymbol{x} \boldsymbol{x} \cdot \mathcal{B}_{0}\right\rangle=\mathbf{0}$, respectively; otherwise they must be of the following form. If $\left\langle\boldsymbol{x} \boldsymbol{x} \cdot \mathcal{B}_{0}\right\rangle \neq \mathbf{0}$, then

$$
\omega_{0}=c \frac{\left\langle\boldsymbol{x} \times \mathcal{E}_{0}\right\rangle \times\left\langle\boldsymbol{x} \boldsymbol{x} \cdot \mathcal{B}_{0}\right\rangle}{\left|\left\langle\boldsymbol{x} \boldsymbol{x} \cdot \mathcal{B}_{0}\right\rangle\right|^{2}}+\gamma\left\langle\boldsymbol{x} \boldsymbol{x} \cdot \boldsymbol{B}_{0}\right\rangle,
$$

which leaves only one degree of freedom to choose from instead of three, viz. the coefficient $\gamma$ is at our disposal. If $\left\langle\boldsymbol{B}_{0}\right\rangle \neq \mathbf{0}$, then

$$
\dot{\boldsymbol{q}}_{0}=\frac{1}{\left|\left\langle\mathcal{B}_{0}\right\rangle\right|^{2}}\left(c\left\langle\mathcal{E}_{0}\right\rangle+\left(\left\langle\boldsymbol{x} \otimes \mathcal{B}_{0}\right\rangle-\left(\operatorname{tr}\left\langle\boldsymbol{x} \otimes \mathcal{B}_{0}\right\rangle\right) \mathbf{I}\right) \cdot \omega_{0}\right) \times\left\langle\mathcal{B}_{0}\right\rangle+\beta\left\langle\mathcal{B}_{0}\right\rangle,
$$

which again leaves only one degree of freedom to choose from instead of three, here the coefficient $\beta$ is at our disposal. The extent to which such consistently restricted initial data now launch a unique regular solution of the system of Abraham equations (A.102), (A.103), (A.78), (A.79), (A.80), and (A.81), with (A.82) and (A.83), is currently not known.

\section{A.3.4 The Abraham model without spin}

The purely electromagnetic Abraham model without spin [79, 80 obtains from the Abraham model with spin by simply discarding (A.103) and setting $\boldsymbol{\omega}=\mathbf{0}$ identically in (A.83), so that (A.102) goes over into

$$
\mathbf{0}=\int_{\mathbb{R}^{3}}\left[\boldsymbol{E}(\boldsymbol{x}, t)+\frac{1}{c} \dot{\boldsymbol{q}}(t) \times \boldsymbol{B}(\boldsymbol{x}, t)\right] f_{\mathrm{e}}(|\boldsymbol{x}-\boldsymbol{q}(t)|) \mathrm{d}^{3} x,
$$

while the Maxwell-Lorentz equations for this model are the same as for the massive model without spin, i.e. again given by (A.78), (A.80), A.79), (A.81), with the charge density (A.82) and with the current density vector (A.93). The continuity equation for the charge is the simpler (A.99), while the law of charge conservation is of course just (A.82). The conserved energy, linear and angular momentum are given again by the purely electromagnetic expressions (A.104), A.105), and (A.106) that apply to the Abraham model with spin. The Abraham model without spin can also be obtained as the singular limit $m_{\mathrm{b}} \rightarrow 0$ of the massive semi-relativistic model without spin under the hypothesis that $\dot{\boldsymbol{q}}$ remains bounded.

For the Abraham model without spin a similar assessment holds as for the Abraham model with spin. Again choosing the initial time to be $t=0$ and the initial particle position to be $\boldsymbol{q}_{0}=\mathbf{0}$ (after at most a time and a space translation), the restrictions for the field data are now determined by (A.107) with $\boldsymbol{\omega}_{0}=\mathbf{0}$ (and ignoring the discarded (A.108)). We conclude that, if $\left\langle\mathcal{B}_{0}\right\rangle=\mathbf{0}$ then also $\left\langle\mathcal{E}_{0}\right\rangle=\mathbf{0}$ must hold, and if $\left\langle\mathcal{B}_{0}\right\rangle \neq \mathbf{0}$, then $\left\langle\mathcal{E}_{0}\right\rangle \cdot\left\langle\mathcal{B}_{0}\right\rangle=0$ must hold. Although these are milder restrictions than those for the Abraham model with spin, these restrictions on the field data in the Abraham model without spin still rule out various perfectly physical initial data, for instance those remarked on in the previous subsection, i.e. $\boldsymbol{q}_{0}=\mathbf{0}, \mathcal{B}_{0}=\mathbf{0}$, and $\mathcal{E}_{0}=\mathcal{E}^{\text {hom }}+\mathcal{E}^{\text {Couloms }}$

As for the initial velocity of the particle $\dot{\boldsymbol{q}}_{0}$ in given consistently restricted initial fields, the following rules hold. The initial velocity $\dot{\boldsymbol{q}}_{0}$ is at our disposal only if $\left\langle\boldsymbol{\mathcal { B }}_{0}\right\rangle=\mathbf{0}$, and if $\left\langle\boldsymbol{B}_{0}\right\rangle \neq \mathbf{0}$ it is of the form

$$
\dot{\boldsymbol{q}}_{0}=c \frac{\left\langle\mathcal{E}_{0}\right\rangle \times\left\langle\mathcal{B}_{0}\right\rangle}{\left|\left\langle\mathcal{B}_{0}\right\rangle\right|^{2}}+\beta\left\langle\mathcal{B}_{0}\right\rangle
$$


which, once again, leaves us only the choice of $\beta$.

Whether such a restricted, consistent set of initial data can now be continued into a unique regular solution of (A.111), A.78), (A.80), (A.79), and (A.81), with (A.82) and (A.93), is currently not rigorously known. As a matter of fact, there are good reasons to doubt that the evolution problem is well-defined even given consistent initial data. For notice that (A.111) states that at

$$
\langle\mathcal{E}\rangle(t)+\frac{1}{c} \dot{\boldsymbol{q}}(t) \times\langle\mathcal{B}\rangle(t)=\mathbf{0},
$$

for all $t$, where the averages here are now defined by

$$
\langle g\rangle(t) \stackrel{\text { def }}{=} \frac{1}{-e} \int_{\mathbb{R}^{3}} g(\boldsymbol{x}, t) f_{\mathrm{e}}(|\boldsymbol{x}-\boldsymbol{q}(t)|) \mathrm{d}^{3} x .
$$

Hence, we can repeat our discussion of the conditions on the initial data essentially verbatim to conclude that:

- If at any time $t$ we have $\langle\mathcal{B}\rangle(t)=\mathbf{0}$, then (A.113) has no solution $\dot{\boldsymbol{q}}(t)$ at that instant $t$ unless also $\langle\mathcal{E}\rangle(t)=\mathbf{0}$;

- If $\langle\boldsymbol{B}\rangle(t) \neq \mathbf{0}$, then (A.113) has no solution $\dot{\boldsymbol{q}}(t)$ at $t$ unless also $\langle\mathcal{E}\rangle(t) \cdot\langle\boldsymbol{B}\rangle(t)=0$;

- If $\langle\boldsymbol{B}\rangle(t) \neq \mathbf{0}$ and $\langle\mathcal{E}\rangle(t) \cdot\langle\mathcal{B}\rangle(t)=0$, then A.113) states that $\dot{\boldsymbol{q}}(t)$ is of the form

$$
\dot{\boldsymbol{q}}(t)=c \frac{\langle\mathcal{E}\rangle(t) \times\langle\boldsymbol{B}\rangle(t)}{|\langle\mathcal{B}\rangle(t)|^{2}}+\beta(t)\langle\mathcal{B}\rangle(t)
$$

with an a priori undetermined coefficient $\beta(t)$.

It does not seem very likely that this system of equations, coupled to the Maxwell-Lorentz equations, evolves a unique regular solution from a consistent set of initial data.

While there is hardly any literature on the Abraham model with spin, the Abraham model without spin is discussed in most major texts since Abraham [2], e.g. [13, 24, 26, 27, 29, 81], and also in the more leisurely text of Peierls [65]. However, none of these texts makes the elementary observation that the initial value problem for the purely electromagnetic Abraham model(s) is singular in the sense explained above, nor that Abraham's 'evolution' equation (A.113) preserves this singular structure in time; some of the main conclusions drawn in 26, 13, 24, 27, 81, 29 are even in open conflict with the singular nature of Abraham's evolu-

tion equations. A re-assessment of these traditional treatments of the Abraham model seems to be called for.

\section{References}

[1] Abraham, M., "Prinzipien der Dynamik des Elektrons," Phys. Z. 4, (1902) 57; Ann. Phys. 10, (1903) 105.

[2] Abraham, M., "Theorie der Elektrizität," vol. II, Teubner, Leipzig, 1905. 
[3] Lorentz, H.A., "Electromagnetic phenomena in a system moving with any velocity less than that of light," Proc. Acad. Wet. Amsterdam, 6 (1904), 809.

[4] Poincaré, H., "Sur la dynamique de l'électron," Comptes-Rendus 140, (1905) 1504; Rendiconti del Circolo Matematico di Palermo 21, (1906) 129.

[5] Miller, A. I., "Albert Einstein's special theory of relativity," Springer, New York, 1998.

[6] Einstein, A., "Zur Elektrodynamik bewegter Körper," Ann. Phys. 17, (1905) 891.

[7] Einstein, A., "Ist die Trägheit eines Körpers von seinem Energieinhalt abhängig?," Ann. Phys. 18, (1905) 639.

[8] Minkowski, H., "Die Grundgleichungen für elektromagnetische Vorgänge in bewegten Körpern," Nachr. Königl. Ges. Wiss. Göttingen, Math. Phys. Kl., (1908) 53.

[9] Uhlenbeck, G.E., and Goudsmit, S.A., "Spinning electrons and the structure of spectra," Nature 117, (1926) 264.

[10] Tomonaga, S., "The story of spin," Univ. Chicago Press, Chicago, 1997.

[11] Glimm, J., and Jaffe, A., "Quantum Physics," 2nd. ed., Springer, New York, 1980.

[12] Fernandez, R., Fröhlich, J., and Sokal, A., "Random Walks, Critical Phenomena, and Triviality in Quantum Field Theory," Springer, New York, 1992.

[13] Lorentz, H.A., "The Theory of electrons and its applications to the phenomena of light and radiant heat," reprint of the 2nd ed., 1915, by Dover, New York, 1952.

[14] Landau, L., and Lifshitz, E.M., "The theory of classical fields," Pergamon Press, Oxford, 1962.

[15] Nodvik, J.S., "A covariant formulation of Classical Electrodynamics for charges of finite extension," Ann. Phys. (N.Y.) 28, (1964) 225.

[16] Misner, C.W., Thorne, K.S., and Wheeler, J.A., "Gravitation," W.H. Freeman Co., New York, 1973.

[17] Streater, R.F., and Wightman, A., "PCT, Spin and Statistics, and All That," Princeton Univ. Press, Princeton, 1964.

[18] Barut, A.O., "Electrodynamics and classical theory of fields and particles," Dover, New York, 1964.

[19] Gittel, H.-P., Kijowski, J., and Zeidler, E., "The relativistic dynamics of the combined particle-field system in renormalized classical electrodynamics," Commun. Math. Phys. 198, (1998) 711.

[20] Thomas, L.H., "The motion of the spinning electron," Nature 117, (1926) 514; "On the kinematics of an electron with an axis," Phil. Mag. 3, (1927) 1. 
[21] Bargmann, V., Michel, L., and Telegdi, V.L., "Precession of the polarization of particles moving in a homogeneous electromagnetic field," Phys. Rev. Lett. 2, (1955) 435.

[22] Fermi, E., "Über einen Widerspruch zwischen der elektrodynamischen und der relativistischen Theorie der elektromagnetischen Masse," Physik. Zeitschr. 23, (1922) 340.

[23] Rohrlich, F., "Self-energy and stability of the classical electron," Am. J. Phys. 28, (1960) 639.

[24] Panofsky, W.K.H., and Phillips, M., "Classical electricity and magnetism," 2nd edition, Addison-Wesley, Reading, Mass., 1962.

[25] Feynman, R.P., Leighton, R.B., and Sands, M., "The Feynman Lectures in Physics," Addison-Wesley, MA, 1963.

[26] Jackson, J.D., "Classical Electrodynamics," 3rd ed., Wiley, New York, 1999.

[27] Rohrlich, F., "Classical charged particles," 2nd. ed., Addison Wesley, Redwood City, CA, 1990.

[28] Schwinger, J., "Electromagnetic mass revisited," Found. Phys. 13, (1983) 373.

[29] Yaghjian, A.D., "Relativistic dynamics of a charged sphere," Lect. Notes Phys. m11, Springer, Berlin, 1992.

[30] Barut, A.O., and Pavšič, "Radiation reaction and the electromagnetic energymomentum of moving relativistic charged membranes," Phys. Lett. B 331, (1994) 45 .

[31] Weinberg, S., "Gravitation and Cosmology: Principles and Applications of the General Theory of Relativity," Wiley, New York, 1972.

[32] Christodoulou, D., "The action principle and partial differential equations," Ann. Math. Stud. 146, Princeton Univ. Press, Princeton, 2000.

[33] Appel, W., and Kiessling, M.K.-H., in preparation for Lett. Math. Phys. (2000).

[34] Hawking, S.W., and Ellis, G.F.R., "The large scale structure of space-time," Cambridge Univ. Press, Cambridge, 1973.

[35] Christodoulou, D. and Klainerman, S., "The global nonlinear stability of the Minkowski space," Princeton Math. Ser. 41, Princeton Univ. Press, Princeton, 1993.

[36] Thorne, K., Price, R.H., and McDonald, D.A., "Black Holes: The membrane paradigm," Yale Univ. Press, Yale, 1986.

[37] Reed, M., and Simon, B., "Scattering theory," (Methods of modern mathematical physics III), Acad. Press, Orlando, 1979. 
[38] Sigal, I.M. and Soffer, A., "The $N$-particle scattering problem: asymptotic completeness for short-range systems," Ann. Math. 126, (1987) 35.

[39] Sigal, I.M. and Soffer, A., "Asymptotic completeness for $N$-particle long-range scattering," J. Am. Math. Soc. 7, (1994) 307.

[40] Dürr, D., Goldstein, S., Teufel, S. and Zanghì, N. "Scattering theory from microscopic first principles," Physica A 279, (2000) 416.

[41] Kunze, M., "Instability of the Periodic Motion of a Particle Interacting with a Scalar Wave Field," Comm. Math. Phys. 195, (1998) 509.

[42] Soffer, A., and Weinstein, M., "Resonances, radiation damping, and instability in Hamiltonian nonlinear wave equations," Inv. Math. 136, (1999) 9.

[43] Spohn, H., "Dynamics of charged particles and their radiation field," Monograph Preprint (131 pages), TU München, 1999.

[44] Cottingham, W.N., "The isolated electron," in "Electron - a centenary volume," (M. Springford, Ed.), p. 24, Cambridge Univ. Press, Cambridge, 1997.

[45] Dyson, F., "The radiation theories of Tomonaga, Schwinger, and Feynman," Phys. Rev. 75, (1949) 486; "The S matrix in Quantum Electrodynamics," ibid. p. 1736.

[46] Dyson, F., "Divergence of perturbation theory in Quantum Electrodynamics," Phys. Rev. 85, (1952) 631.

[47] Balaban, T., private communications (1999).

[48] Wightman, A. private communication (Dec. 1999).

[49] Wilczek, F., "The future of particle physics as a natural science," in "Critical Problems in Physics," (V. Fitch, D. Marlow, M. Dementi, Eds.), p. 281, Princeton Univ. Press, Princeton 1997.

[50] Witten, E., "Vistas in Theoretical Physics," in "Critical Problems in Physics," (V. Fitch, D. Marlow, M. Dementi, Eds.), p. 271, Princeton Univ. Press, Princeton 1997.

[51] Connes, A., "Noncommutative Geometry," Academic Press, New York, 1994.

[52] Schweber, S., "QED and the man who made it: Dyson, Feynman, Schwinger, and Tomonaga," Princeton Univ. Press, Princeton 1994.

[53] Griesemer, M. Lieb, E.H., and Loss, M., "Ground states in non-relativistic QED," LANL e-print arXive math-ph/0007014 (Jul. 2000).

[54] Kontsevich, M., "Deformation quantization of Poisson manifolds," LANL e-print arXive //http://xxx.lanl.gov/q-alg/9709040 (1997).

[55] Nyborg, P., "On classical theories of spinning particles," Nuovo Cim. 23, (1962) 47. 
[56] Benguria, R., "Electrodynamics and the equations of motion for charged particles with spin," Phys. Rev. D. 13, (1976) 274.

[57] Glassey, R., and Schaeffer, J., "Global existence for the relativistic Vlasov-Maxwell system with nearly neutral initial data," Comm. Math. Phys. 119, (1988) 353.

[58] Glassey, R., and Strauss, W., "Absence of shocks in an initially dilute collisionless plasma," Comm. Math. Phys. 113, (1987) 191.

[59] Glassey, R., and Strauss, W. "Singularity formation in a collisionless plasma could occur only at high velocities," Arch. Rat. Mech. Anal. 92, (1986) 59.

[60] Vlasov, A.A., "Many-particle theory and its application to plasma," Gordon and Breach, New York, 1961; published originally by State Publishing House for TechnicalTheoretical Literature, Moscow and Leningrad, 1950.

[61] Dirac, P.A.M., "Classical theory of radiating electrons," Proc. Roy. Soc. A 167, (1938) 148.

[62] Carati, A., Delzanno, P., Galgani, L., and Sassarini, J., "Nonuniqueness properties of the physical solutions of the Lorentz-Dirac equation," Nonlinearity 8, (1995) 65.

[63] Gaftoi, V., López-Bonilla, J. L., Morales, J., and Rosales, M. A., "The Lorentz-Dirac equation in Minkowski space," J. Math. Phys. 35, (1994) 3482.

[64] Luca, J. De, "The Lorentz-Dirac equation for linear and nonlinear potentials," Braz. J. Phys. 27, (1997) 285.

[65] Peierls, R., "More surprises in theoretical physics," Princeton Univ., Princeton, 1991.

[66] Bambusi, D., and Noja, D., "Some rigorous results on the Pauli-Fierz model of classical electrodynamics," Lett. Math Phys. 37, (1996) 449.

[67] Bambusi, D., and Galgani, L., "Some rigorous results on the Pauli-Fierz model of classical electrodynamics," Ann. Inst. H. Poincaré, Phys. Theor. 58, (1993) 155.

[68] Noja, D., and Posilicano, A., "On the point limit of the Pauli-Fierz model," Ann. Inst. H. Poincaré, Phys. Theor. 71, (1999) 425.

[69] Noja, D., and Posilicano, A., "Delta interactions and electrodynamics of point particles," Los Alamos e-print //http://xxx.lanl.gov/math-ph/9907009 (1999).

[70] Lorentz, H.A., "La théorie électromagnetique de Maxwell et son application aux corps mouvants," Arch. Néerl. Sci. Exactes Nat. 25, (1892) 363.

[71] Kiessling, M. K.-H., "Classical electron theory and conservation laws," Phys. Lett. A 258, (1999) 197.

[72] Bauer, G., and Dürr, D., "The Maxwell-Lorentz system of a rigid charge distribution," Preprint, LMU München, 1999. 
[73] Komech, A., and Spohn, H., "Long-time asymptotics for the coupled Maxwell-Lorentz Equations," Commun. PDE 25, (2000) 559.

[74] Kunze, M., and Spohn, H., "Adiabatic limit of the Maxwell-Lorentz equations," Ann. Inst. H. Poincaré, Phys. Théor., (2000) in press.

[75] Kunze, M., and Spohn, H., "Radiation reaction and center manifolds," SIAM J. Math. Anal., (2000) in press.

[76] Kunze, M., and Spohn, H., Slow motion of charges interacting through the Maxwell field," Commun. Math. Phys. 212, (2000) 437.

[77] Komech, A., Spohn, H., and Kunze, M., "Long-time asymptotics for a classical particle interacting with a scalar wave field," Commun. PDE 22, (1997) 307.

[78] Komech, A., Kunze, M., and Spohn, H., "Effective dynamics for a mechanical particle coupled to a wave field," Commun. Math. Phys. 203, (1999) 1.

[79] Lorentz, H.A., "Versuch einer Theorie der elektrischen und optischen Erscheinungen in bewegten Körpern," Teubner, Leipzig, 1909; orig.: Brill, Leyden, 1895.

[80] Lorentz, H.A., "Weiterbildung der Maxwell'schen Theorie: Elektronentheorie.," in Encyklopädie d. Mathematischen Wissenschaften V2, Art. 14, p. 145, 1904.

[81] Sommerfeld, A., "Electrodynamics," Academic Press, New York, 1952. 